\title{
Precision tests of the Standard Model with leptonic and semileptonic kaon decays
}

\author{
The FlaviaNet Kaon Working Group* ${ }^{* \ddagger}$
}

ABSTRACT: We present a global analysis of leptonic and semileptonic kaon decays data, including all recent results by BNL-E865, KLOE, KTeV, ISTRA+, and NA48. Experimental results are critically reviewed and combined, taking into account theoretical (both analytical and numerical) constraints on the semileptonic kaon form factors. This analysis leads to a very accurate determination of $V_{u s}$ and allows us to perform several stringent tests of the Standard Model.

KeYwords: Vus, CKM, Kaon.

${ }^{*}$ WWW access at http://www.lnf.infn.it/wg/vus

${ }^{\dagger}$ The members of the FlaviaNet Kaon Working Group who contributed more significantly to this note are: M. Antonelli, V. Cirigliano, P. Franzini, S Glazov, R. Hill, G. Isidori, F. Mescia, M. Moulson, M. Palutan, E. Passemar, M. Piccini, M. Veltri, O. Yushchenko, R. Wanke.

${ }^{\ddagger}$ The Collaborations each take responsibility for the preliminary results of their own experiment. 


\section{Contents}

1. Introduction 2

2. Theoretical framework 3

2.1 $K_{\ell 3}$ and $K_{\ell 2}$ rates within the SM 3

2.2 Parametrization of $K_{\ell 3}$ form factors 4

2.2.1 Dispersive constraints 5

$\begin{array}{ll}2.2 .2 & \text { Analyticity and improved series expansion }\end{array}$

$2.3 \quad K_{\ell 3}$ and $K_{\ell 2}$ decays beyond the SM 8

2.3.1 The $s \rightarrow u$ effective Hamiltonian 8

$\begin{array}{lll}2.3 .2 & K_{\ell 2} \text { rates } & 10\end{array}$

$\begin{array}{lll}2.3 .3 & K_{\ell 3} \text { rates and kinematical distributions } & 10\end{array}$

3. Data Analysis 12

$3.1 K_{L}$ leading branching ratios and $\tau_{L} \quad 12$

$3.2 K_{S}$ leading branching ratios and $\tau_{S} \quad 14$

$\begin{array}{lll}3.3 & K^{ \pm} \text {leading branching ratios and } \tau^{ \pm} & 14\end{array}$

3.4 Measurement of $\operatorname{BR}\left(K_{e 2}\right) / \operatorname{BR}\left(K_{\mu 2}\right) \quad 15$

3.5 Measurements of $\boldsymbol{K}_{\ell \mathbf{3}}$ slopes 16

3.5.1 Vector form factor slopes from $\boldsymbol{K}_{\boldsymbol{\ell} \mathbf{3}} \quad 16$

$\begin{array}{ll}3.5 .2 & \text { Scalar and Vector form factor slopes from } \boldsymbol{K}_{\ell \mathbf{3}} \\ \end{array}$

4. Physics Results 20

4.1 Determination of $\left|V_{u s}\right| \times f_{+}(0)$ and $\left|V_{u s}\right| /\left|V_{u d}\right| \times f_{K} / f_{\pi} \quad 20$

4.1.1 Determination of $\left|V_{u s}\right| \times f_{+}(0) \quad 20$

4.1.2 Determination of $\left|V_{u s}\right| /\left|V_{u d}\right| \times f_{K} / f_{\pi} \quad 21$

4.2 The parameters $f_{+}(0)$ and $f_{K} / f_{\pi} \quad 21$

4.2.1 Theoretical estimates of $f_{+}(0) \quad 21$

4.2.2 Theoretical estimates of $f_{K} / f_{\pi} \quad 24$

4.2.3 A test of lattice calculation: the Callan-Treiman relation 24

4.3 Test of Cabibbo Universality or CKM unitarity 27

4.3.1 Bounds on helicity-suppressed amplitudes 28

4.4 Tests of Lepton Flavor Universality 30

4.4.1 Lepton universality in $K_{\ell 3}$ decays 30

4.4.2 Lepton universality tests in $K_{\ell 2}$ decays 30

$\begin{array}{ll}\text { Acknowledgments } & 31\end{array}$

$\begin{array}{ll}\text { A. BRS fit procedure } & 36\end{array}$ 
B. Fit for $K_{L}$ BRs and lifetime

B.1 Results 39

G. Fit for $K^{ \pm}$BRs and lifetime $\quad 41$

C.1 Results 43

D. Averages of form-factor slopes 44

D.1 Procedure 44

D.2 Input data 44

D.3 Fit results for $\boldsymbol{K}_{\boldsymbol{\ell} \mathbf{3}}$ slopes excluding NA48 $\boldsymbol{K}_{\boldsymbol{\mu} \mathbf{3}}$ data 46

E. Error estimates $\quad 47$

$\begin{array}{lll}\text { E.1 } K_{e 3} \text { decays } & 47\end{array}$

E.2 $K_{\mu 3}$ decays 48

E.3 From the linear to the dispersive parametrization 48

\section{Introduction}

In the Standard Model, SM, transition rates of semileptonic processes such as $d^{i} \rightarrow u^{j} \ell \nu$, with $d^{i}\left(u^{j}\right)$ being a generic down (up) quark, can be computed with high accuracy in terms of the Fermi coupling $G_{F}$ and the elements $V_{j i}$ of the Cabibbo-Kobayashi Maskawa (CKM) matrix [1]. Measurements of the transition rates provide therefore precise determinations of the fundamental SM couplings.

A detailed analysis of semileptonic decays offers also the possibility to set stringent constraints on new physics scenarios. While within the SM all $d^{i} \rightarrow u^{j} \ell \nu$ transitions are ruled by the same CKM coupling $V_{j i}$ (satisfying the unitarity condition $\sum_{k}\left|V_{i k}\right|^{2}=1$ ) and $G_{F}$ is the same coupling appearing in the muon decay, this is not necessarily true beyond the SM. Setting bounds on the violations of CKM unitarity, violations of lepton universality, and deviations from the $V-A$ structure, allows us to put significant constraints on various new-physics scenarios (or eventually find evidences of new physics).

In the case of leptonic and semileptonic $K$ decays these tests are particularly significant given the large amount of data recently collected by several experiments: BNL-E865, KLOE, KTeV, ISTRA+, and NA48. These data allow to perform very stringent SM tests which are almost free from hadronic uncertainties (such as the $\mu / e$ universality ratio in $K_{\ell 2}$ decays). In addition, the high statistical precision and the detailed information on kinematical distributions have stimulated a substantial progress also on the theory side: most of the theory-dominated errors associated to hadronic form factors have recently been reduced below the $1 \%$ level.

An illustration of the importance of semileptonic $K$ decays in testing the $\mathrm{SM}$ is provided by the unitarity relation

$$
\left|V_{u d}\right|^{2}+\left|V_{u s}\right|^{2}+\left|V_{u b}\right|^{2}=1+\epsilon_{\mathrm{NP}}
$$


Here the $V_{j i}$ are the CKM elements determined from the various $d^{i} \rightarrow u^{j}$ processes, having fixed $G_{F}$ from the muon life time: $G_{\mu}=1.166371(6) \times 10^{-5} \mathrm{GeV}^{-2}$ [2]. $\epsilon_{\mathrm{NP}}$ parametrizes possible deviations from the SM induced by dimension-six operators, contributing either to the muon decay or to the $d^{i} \rightarrow u^{j}$ transitions. By dimensional arguments we expect $\epsilon_{\mathrm{NP}} \sim M_{W}^{2} / \Lambda_{\mathrm{NP}}^{2}$, where $\Lambda_{\mathrm{NP}}$ is the effective scale of new physics. The present accuracy on $\left|V_{u s}\right|$, which is the dominant source of error in (1.1), allows to set bounds on $\epsilon_{\mathrm{NP}}$ around $0.1 \%$ or equivalently to set bounds on the new physics scale well above $1 \mathrm{TeV}$.

In this note we report on progress in the verification of the relation (1.1) as well as on many other tests of the SM which can be performed with leptonic and semileptonic $K$ decays. The note is organized as follows. The phenomenological framework needed to describe $K_{\ell 3}$ and $K_{\mu 2}$ decays within and beyond the SM is briefly reviewed in Section 2. Section 3 is dedicated to the combination of the experimental data. The results and the interpretation are presented in Section 6 .

\section{Theoretical framework}

\section{$2.1 K_{\ell 3}$ and $K_{\ell 2}$ rates within the SM}

Within the SM the photon-inclusive $K_{\ell 3}$ and $K_{\ell 2}$ decay rates are conveniently decomposed as [3]

$$
\begin{aligned}
& \Gamma\left(K_{\ell 3(\gamma)}\right)=\frac{G_{F}^{2} m_{K}^{5}}{192 \pi^{3}} C_{K} S_{\mathrm{ew}}\left|V_{u s}\right|^{2} f_{+}(0)^{2} I_{K}^{\ell}\left(\lambda_{+, 0}\right)\left(1+\delta_{S U(2)}^{K}+\delta_{\mathrm{em}}^{K \ell}\right)^{2} \\
& \frac{\Gamma\left(K_{\ell 2(\gamma)}^{ \pm}\right)}{\Gamma\left(\pi_{\ell 2(\gamma)}^{ \pm}\right)}=\left|\frac{V_{u s}}{V_{u d}}\right|^{2} \frac{f_{K}^{2} m_{K}}{f_{\pi}^{2} m_{\pi}}\left(\frac{1-m_{\ell}^{2} / m_{K}^{2}}{1-m_{\ell}^{2} / m_{\pi}^{2}}\right)^{2} \times\left(1+\delta_{\mathrm{em}}\right)
\end{aligned}
$$

where $C_{K}=1(1 / 2)$ for the neutral (charged) kaon decays, $I_{K}^{\ell}\left(\lambda_{+, 0}\right)$ is the phase space integral that depends on the (experimentally accessible) slopes of the form factors (generically denoted by $\lambda_{+, 0}$ ), and $S_{\text {ew }}=1.0232(3)$ is the universal short-distance electromagnetic correction computed in Ref. [⿴囗十⺝刂. The channel-dependent long-distance electromagnetic correction factors are denoted by $\delta_{\mathrm{em}}$ and $\delta_{\mathrm{em}}^{K \ell}$. In the $K_{\ell 2}$ case $\delta_{\mathrm{em}}=-0.0070(35)$ [5, 6], while the four $\delta_{\mathrm{em}}^{K \ell}$ are given in Table 1, together with the isospin-breaking corrections due to $m_{u} \neq m_{d}$, denoted by $\delta_{S U(2)}^{K}$.

The overall normalization of the $K_{\ell 3}$ rates depends upon $f_{+}(0)$, the $K \rightarrow \pi$ vector form factor at zero momentum transfer $\left[t=\left(p_{K}-p_{\pi}\right)^{2}=0\right]$. By convention, $f_{+}(0)$ is defined for the $K^{0} \rightarrow \pi^{-}$matrix element, in the limit $m_{u}=m_{d}$ and $\alpha_{\mathrm{em}} \rightarrow 0$ (keeping kaon and pion masses to their physical value). Similarly, $f_{K} / f_{\pi}$ is the ratio of the kaon and pion decay constants defined in the $m_{u}=m_{d}$ and $\alpha_{\mathrm{em}} \rightarrow 0$ limit. The values of these hadronic parameters, which represent the dominant source of theoretical uncertainty, will be discussed in Sect. 4.2.

The errors for the $K_{\ell 3}$ electromagnetic corrections, given in Table 1, have been obtained within ChPT, estimating higher-order corrections by naive dimensional analysis [7, 8]. Higher-order chiral corrections have a minor impact in the breaking of lepton universality. 


\begin{tabular}{c||c||c|} 
& $\delta_{S U(2)}^{K}(\%)$ & $\delta_{\mathrm{em}}^{K \ell}(\%)$ \\
\hline$K_{e 3}^{0}$ & 0 & $+0.57(15)$ \\
$K_{e 3}^{+}$ & $2.36(22)$ & $+0.08(15)$ \\
$K_{\mu 3}^{0}$ & 0 & $+0.80(15)$ \\
$K_{\mu 3}^{+}$ & $2.36(22)$ & $+0.05(15)$
\end{tabular}

Table 1: Summary of the isospin-breaking corrections factors [7, 8]. The electromagnetic corrections factors correspond to the fully-inclusive $K_{\ell 3(\gamma)}$ rate.

The errors are correlated as given below:

$$
\left(\begin{array}{cccc}
1.0 & 0.1 & 0.8 & -0.1 \\
& 1.0 & -0.1 & 0.8 \\
& & 1.0 & 0.1 \\
& & & 1.0
\end{array}\right) .
$$

\subsection{Parametrization of $K_{\ell 3}$ form factors}

The hadronic $K \rightarrow \pi$ matrix element of the vector current is described by two form factors (FFs), $f_{+}(t)$ and $f_{0}(t)$, defined by

$$
\begin{aligned}
\left\langle\pi^{-}(k)\left|\bar{s} \gamma^{\mu} u\right| K^{0}(p)\right\rangle & =(p+k)^{\mu} f_{+}(t)+(p-k)^{\mu} f_{-}(t) \\
f_{-}(t) & =\frac{m_{K}^{2}-m_{\pi}^{2}}{t}\left(f_{0}(t)-f_{+}(t)\right)
\end{aligned}
$$

where $t=(p-k)^{2}$. By construction, $f_{0}(0)=f_{+}(0)$.

In order to compute the phase space integrals appearing in Eq. (2.1) we need experimental or theoretical inputs about the $t$-dependence of $f_{+, 0}(t)$. In principle, Chiral Perturbation Theory (ChPT) and Lattice QCD are useful tools to set theoretical constraints. However, in practice the $t$-dependence of the FFs at present is better determined by measurements and by combining measurements and dispersion relations.

In the physical region, $\left(m_{\ell}^{2}<t<\left(m_{K}-m_{\pi}\right)^{2}\right)$, a very good approximation for the FFs is given by a Taylor expansion up to $t^{2}$ terms

$$
\tilde{f}_{+, 0}(t) \equiv \frac{f_{+, 0}(t)}{f_{+}(0)}=1+\lambda_{+, 0}^{\prime} \frac{t}{m_{\pi}^{2}}+\frac{1}{2} \lambda_{+, 0}^{\prime \prime}\left(\frac{t}{m_{\pi}^{2}}\right)^{2}+\ldots
$$

Note that $t=\left(p_{K}-p_{\pi}\right)^{2}=m_{K}^{2}+m_{\pi}^{2}-2 m_{K} E_{\pi}$, therefore the FFs depend only on $E_{\pi}$. The FF parameters can thus be obtained from a fit to the pion spectrum which is of the form $g\left(E_{\pi}\right) \times \tilde{f}\left(E_{\pi}\right)^{2}$. Unfortunately $t$ is maximum for $E_{\pi}=0$, where $g\left(E_{\pi}\right)$ vanishes.

Still, experimental information about the vector form factor $\tilde{f}_{+}$measured both from $K_{e 3}$ and $K_{\mu 3}$ data are quite accurate and so far superior to theoretical predictions. A pole parametrization, $\tilde{f}_{+}(t)=M_{V}^{2} /\left(M_{V}^{2}-t\right)$, with $M_{V} \sim 892 \mathrm{MeV}$ corresponding to the $K^{*}(892)$ resonance and which predicts $\lambda_{+}^{\prime \prime}=2\left(\lambda_{+}^{\prime}\right)^{2}$, is in good agreement with present data (see later). Improvements of this parametrization have been proposed in Refs. [9, 10, 11]. For instance, in Ref. [11], a dispersive parametrization for $\tilde{f}_{+}$, which has good analytical and unitarity properties and a correct threshold behavior, has been built. 
The situation for the scalar form factor $\tilde{f}_{0}(t)$ is more complex. For kinematical reasons $f_{0}(t)$ is only accessible from $K_{\mu 3}$ data and one has to deal with the correlations between the two form factors. Moreover, for $f_{0}(t)$, the curvature $\lambda_{0}^{\prime \prime}$ cannot be determined from the data and different assumptions for the parametrization of $\tilde{f}_{0}$ such as linear, quadratic or polar lead to different results for the slope $\lambda_{0}^{\prime}$ which cannot be discriminated from the data alone. In turn, these ambiguities induce a systematic uncertainty for $V_{u s}$, even though data for partial rates by itself are very accurate. For this reason, the parametrization used has to rely on theoretical arguments being as model-independent as possible and allowing to measure at least the slope and the curvature of the form factor.

\subsubsection{Dispersive constraints}

The vector and scalar form factors $f_{+, 0}(t)$ in Eq. (2.4) are analytic functions in the complex $t$-plane, except for a cut along the positive real axis, starting at the first physical threshold $t_{\mathrm{th}}=\left(m_{K}+m_{\pi}\right)^{2}$, where they develop discontinuities. They are real for $t<t_{\mathrm{th}}$.

Cauchy's theorem implies that $f_{+, 0}(t)$ can be written as a dispersive integral along the physical cut

$$
f_{+, 0}(t)=\frac{1}{\pi} \int_{t_{\mathrm{th}}}^{\infty} d s^{\prime} \frac{\operatorname{Im} f_{+, 0}\left(s^{\prime}\right)}{\left(s^{\prime}-t-i 0\right)}+\text { subtractions }
$$

where all possible on-shell intermediate states contribute to its imaginary part $\operatorname{Im} F_{k}\left(s^{\prime}\right)$. A number of subtractions is needed to make the integral convergent. Particularly appealing is an improved dispersion relation recently proposed in Ref. [12] where two subtractions are performed at $t=0$ (where by definition, $\tilde{f}_{0}(0) \equiv 1$ ) and at the so-called Callan-Treiman point $t_{C T} \equiv\left(m_{K}^{2}-m_{\pi}^{2}\right)$ leading to

$$
\begin{aligned}
\tilde{f}_{0}(t) & =\exp \left[\frac{t}{t_{C T}}\left(\ln \left(\tilde{f}_{0}\left(t_{C T}\right)\right)-G(t)\right)\right] \\
\text { with } G(t) & =\frac{t_{C T}\left(t_{C T}-t\right)}{\pi} \int_{t_{\mathrm{th}}}^{\infty} \frac{d s^{\prime}}{s^{\prime}} \frac{\phi\left(s^{\prime}\right)}{\left(s^{\prime}-t_{C T}\right)\left(s^{\prime}-t-i \epsilon\right)},
\end{aligned}
$$

assuming that $\tilde{f}_{0}(t)$ has no zero. Here $\phi(x)$, the phase of $\tilde{f}_{0}(t)$, can be identified in the elastic region with the S-wave, $I=1 / 2 K \pi$ scattering phase, $\delta_{K \pi}(s)$, according to Watson theorem.

A subtraction at $t_{C T}$ has been performed because the Callan-Treiman theorem implies

$$
\tilde{f}_{0}\left(t_{C T}\right)=\frac{f_{K}}{f_{\pi}} \frac{1}{f_{+}(0)}+\Delta_{C T}
$$

where $\Delta_{C T} \sim \mathcal{O}\left(m_{u, d} / 4 \pi F_{\pi}\right)$ is a small quantity. ChPT estimates at NLO in the isospin limit [15], obtain

$$
\Delta_{C T}=(-3.5 \pm 8) \times 10^{-3},
$$

where the error is a conservative estimate of the high-order corrections to the expansion in light quark masses [16]. A complete two-loop evaluation of $\Delta_{C T}$, consistent with this estimate, has been recently presented in Ref. [17]. 
Hence, with only one parameter, $\tilde{f}_{0}\left(t_{C T}\right)$, one can determine the shape of $\tilde{f}_{0}$ by fitting the $K_{\mu 3}$ decay distribution with the dispersive representation of $\tilde{f}_{0}(t)$, Eq. (2.7). Then, we can deduce from Eq. (2.7) the three first coefficients of the Taylor expansion, Eq. (2.5), see Ref. [12]:

$$
\begin{aligned}
\lambda_{0}^{\prime}=\frac{m_{\pi}^{2}}{\Delta_{K \pi}} & {\left.\left[\ln \left(\tilde{f}_{0}\left(t_{C T}\right)\right)-G(0)\right]=\frac{m_{\pi}^{2}}{\Delta_{K \pi}}\left[\ln \left(\tilde{f}_{0}\left(t_{C T}\right)\right)-0.0398(40)\right)\right], } \\
\lambda_{0}^{\prime \prime} & =\left(\lambda_{0}^{\prime}\right)^{2}-2 m_{\pi}^{4} / t_{C T} G^{\prime}(0)=\left(\lambda_{0}^{\prime}\right)^{2}+(4.16 \pm 0.50) \times 10^{-4} \\
\lambda_{0}^{\prime \prime \prime} & =\left(\lambda_{0}^{\prime}\right)^{3}-6 m_{\pi}^{4} / t_{C T} G^{\prime}(0) \lambda_{0}^{\prime}-3 m_{\pi}^{6} / t_{C T} G^{\prime \prime}(0) \\
& =\left(\lambda_{0}^{\prime}\right)^{3}+3(4.16 \pm 0.50) \times 10^{-4} \lambda_{0}^{\prime}+(2.72 \pm 0.11) \times 10^{-5}
\end{aligned}
$$

Furthermore, thanks to Eq. (2.8), measuring $\tilde{f}_{0}\left(t_{C T}\right)$ provides a significant constraint on $f_{K} / f_{\pi} / f_{+}(0)$ limited only by the small theoretical uncertainty on $\Delta_{C T}$. As we will discuss in Section 4.2.3, this represents a powerful consistency check of present lattice QCD estimates of $f_{K} / f_{\pi}$ and $f_{+}(0)$.

A similar dispersive parametrization for the vector form factor has been proposed in Ref. [11] with two subtractions performed at $t=0$. This leads to:

$$
\tilde{f}_{+}(t)=\exp \left[\frac{t}{m_{\pi}^{2}}\left(\Lambda_{+}+H(t)\right)\right], \text { where } H(t)=\frac{m_{\pi}^{2} t}{\pi} \int_{t_{K \pi}}^{\infty} \frac{d s}{s^{2}} \frac{\varphi(s)}{(s-t-i \epsilon)} .
$$

In the elastic region, the phase of the vector form factor, $\varphi(s)$, equals the $I=1 / 2, \mathrm{P}$-wave $K \pi$ scattering phase.

Additional tests can be performed using the expression for the scalar form factor $f_{0}(t)$ at order $p^{6}$ in ChPT [18]:

$$
f_{0}(t)=f_{+}(0)+\bar{\Delta}(t)+\frac{\left(f_{K} / f_{\pi}-1\right)}{m_{K}^{2}-m_{\pi}^{2}} t+\frac{8}{f_{\pi}^{4}}\left(2 C_{12}^{r}+C_{34}^{r}\right)\left(m_{K}^{2}+m_{\pi}^{2}\right) t-\frac{8}{f_{\pi}^{4}} C_{12}^{r} t^{2},
$$

where

$$
\begin{aligned}
f_{+}(0) & =1+\Delta(0)-\frac{8}{f_{\pi}^{4}}\left(C_{12}^{r}+C_{34}^{r}\right)\left(m_{K}^{2}-m_{\pi}^{2}\right)^{2} \\
\lambda_{0}^{\prime} & =8 \frac{m_{\pi}^{2}\left(m_{\pi}^{2}+m_{K}^{2}\right)}{f_{\pi}^{4} f_{+}(0)}\left(2 C_{12}^{r}+C_{34}^{r}\right)+\frac{m_{\pi}^{2}}{m_{K}^{2}-m_{\pi}^{2}}\left(\frac{f_{K}}{f_{\pi}} \frac{1}{f_{+}(0)}-\frac{1}{f_{+}(0)}\right)+m_{\pi}^{2} \frac{\bar{\Delta}^{\prime}(0)}{f_{+}(0)} \\
\lambda_{0}^{\prime \prime} & =-16 \frac{m_{\pi}^{4}}{f_{\pi}^{4} f_{+}(0)} C_{12}^{r}+m_{\pi}^{4} \frac{\bar{\Delta}^{\prime \prime}(0)}{f_{+}(0)}
\end{aligned}
$$

Here $\bar{\Delta}(t)$ is a function which receives contributions from order $p^{4}$ and $p^{6}$, but like $\Delta(0)$ it is independent of the $C_{i}^{r}$, and the order $p^{4}$ chiral constants $L_{i}^{r}$ only appear at order $p^{6} . \bar{\Delta}(t)$ and $\Delta(0)$ have been evaluated in the physical region in Ref. [18] using for the $L_{i}^{r}$ values a fit to experimental data. An analysis has been presented in ref. 19. However, the fit has to be reconsidered in light of the new experimental results as for instance considering the new $K_{\ell 4}$ analysis from NA48 and the updated value of $f_{K} / f_{\pi}$. 


\subsubsection{Analyticity and improved series expansion}

Armed only with the knowledge that the form factor is analytic outside the cut on the real axis, analyticity provides powerful constraints on the form factor shape without recourse to model assumptions. In particular, by an appropriate conformal mapping, the series expansion (2.5) necessarily "resums" into the form

$$
f(t)=\frac{1}{\phi}\left(a_{0}+a_{1} z+a_{2} z^{2}+\ldots\right),
$$

where $\phi$ is an analytic function and

$$
z\left(t, t_{0}\right)=\frac{\sqrt{t_{t h}-t}-\sqrt{t_{t h}-t_{0}}}{\sqrt{t_{t h}-t}+\sqrt{t_{t h}-t_{0}}}
$$

is the new expansion parameter. In this " $z$ expansion", the factor $z\left(t, t_{0}\right)$ sums an infinite number of terms, transforming the original series, naively an expansion involving $t / t_{+} \lesssim$ 0.3 , into a series with a much smaller expansion parameter. For example, the choice

$t_{0}=t_{t h}\left(1-\sqrt{1-\left(m_{K}^{2}-m_{\pi}^{2}\right) / t_{t h}}\right)$ minimizes the maximum value of $z$ occurring in the physical region, and for this choice $\left|z\left(t, t_{0}\right)\right| \lesssim 0.047$.

The function $\phi$ and the number $t_{0}$ may be regarded as defining a "scheme" for the expansion. The expansion parameter $z$ and coefficients $a_{k}$ are then "scheme-dependent" quantities, with the scheme dependence dropping out in physical observables such as $f(t)$. For the vector form factor, a convenient choice for $\phi$ is

$$
\begin{aligned}
& \phi_{F_{+}}\left(t, t_{0}, Q^{2}\right)=\sqrt{\frac{1}{32 \pi}} \frac{z(t, 0)}{-t}\left(\frac{z\left(t,-Q^{2}\right)}{-Q^{2}-t}\right)^{3 / 2} \\
& \times\left(\frac{z\left(t, t_{0}\right)}{t_{0}-t}\right)^{-1 / 2}\left(\frac{z\left(t, t_{-}\right)}{t_{-}-t}\right)^{-3 / 4} \frac{t_{+}-t}{\left(t_{+}-t_{0}\right)^{1 / 4}} .
\end{aligned}
$$

This choice is motivated by arguments of unitarity, whereby the coefficients can be bounded by calculating an inclusive production rate in perturbation theory [23]. In fact, a much more stringent bound is obtained by isolating the exclusive $K \pi$ production rate in the vector channel from $\tau$ decay data [22]. This enforces [20]

$$
\sum_{k=0}^{\infty} \frac{a_{k}^{2}}{a_{0}^{2}} \lesssim 170 .
$$

With this choice of $\phi$, and $Q^{2}=2 \mathrm{GeV}^{2}$, a convenient choice for $t_{0}$ is $t_{0}=0.39\left(m_{K}-m_{\pi}\right)^{2}$. This choice eliminates correlations in shape parameters $a_{1} / a_{0}$ and $a_{2} / a_{0}$.

The bound on the expansion coefficients can be used to bound errors on physical quantities describing the form factor shape, as discussed below in Sect. 3.5. A similar expansion can be used for the scalar form factor. Note that error estimates based on (2.19) are conservative - no single coefficient is likely to saturate the bound. Also, this bound is a maximum taken over different schemes; more stringent bounds for particular schemes can be found in 20]. 
In addition to the direct applications in $K_{\ell 3}$ decays, it is important for other purposes to constrain the first few coefficients in (2.16), and check whether the series converges as expected. $K_{\ell 3}$ decays provide a unique opportunity to do this. For example, the same parameterization can be used to constrain the form factor shape in lattice calculations of $f(0)$, with the threshold $t_{t h}$ adjusted to the appropriate value for the simulated quark masses. Measurements of $a_{k}$ in the kaon system can similarly be used to confirm scaling arguments that apply also in the charm and bottom systems [21].

\section{3 $K_{\ell 3}$ and $K_{\ell 2}$ decays beyond the SM}

\subsubsection{The $s \rightarrow u$ effective Hamiltonian}

On general grounds, assuming only Lorentz invariance and neglecting effective operators of dimension higher than six, $\Delta S=1$ charged-current transitions are described by 10 independent operators:

$$
\begin{aligned}
H_{s u}^{\Delta S=1}=-\frac{G_{F}}{\sqrt{2}} & V_{u s}\left[c_{L L}^{V}\left(\bar{s} \gamma^{\mu} L u\right)\left(\bar{\nu} \gamma^{\mu} L \ell\right)+c_{L R}^{V}\left(\bar{s} \gamma^{\mu} L u\right)\left(\bar{\nu} \gamma^{\mu} R \ell\right)\right. \\
& +c_{R L}^{V}\left(\bar{s} \gamma^{\mu} R u\right)\left(\bar{\nu} \gamma^{\mu} L \ell\right)+c_{R R}^{V}\left(\bar{s} \gamma^{\mu} R u\right)\left(\bar{\nu} \gamma^{\mu} R \ell\right) \\
& +c_{L L}^{S}(\bar{s} L u)(\bar{\nu} L \ell)+c_{L R}^{S}(\bar{s} L u)(\bar{\nu} R \ell) \\
& +c_{R L}^{S}(\bar{s} R u)(\bar{\nu} L \ell)+c_{R R}^{S}(\bar{s} R u)(\bar{\nu} R \ell) \\
& \left.+c_{L L}^{T}\left(\bar{s} \sigma^{\mu \nu} L u\right)\left(\bar{\nu} \sigma^{\mu \nu} L \ell\right)+c_{R R}^{T}\left(\bar{s} \sigma^{\mu \nu} R u\right)\left(\bar{\nu} \sigma^{\mu \nu} R \ell\right)\right]+ \text { h.c. }
\end{aligned}
$$

where $L=\left(1-\gamma_{5}\right)$ and $R=\left(1+\gamma_{5}\right)$. Defining this Hamiltonian at the weak scale, the SM case corresponds to $c_{L L}^{V}\left(M_{W}^{2}\right)=1$ and all the other coefficients set to zero. The universal electromagnetic correction factor $S_{\text {ew }}$ appearing in Eq. (2.1) describes the evolution of $c_{L L}^{V}$ to hadronic scales: $c_{L L}^{V}\left(M_{\rho}^{2}\right) / c_{L L}^{V}\left(M_{W}^{2}\right)=1+\left(S_{\mathrm{ew}}-1\right) / 2 \approx S_{\mathrm{ew}}^{1 / 2}$. A similar expression can also be written for the Hamiltonian regulating $u \rightarrow d$ transitions.

In the case of $K \rightarrow \pi \ell \nu$ decays only six independent combinations of these operators have a non-vanishing tree-level matrix element:

$$
\begin{aligned}
\mathcal{A}(K \rightarrow \pi \ell \nu)= & \frac{G_{F}}{\sqrt{2}} V_{u s}\langle\pi \ell \nu| c_{V}\left(\bar{s} \gamma^{\mu} u\right)\left(\bar{\nu} \gamma_{\mu} \ell\right)+c_{A}\left(\bar{s} \gamma^{\mu} u\right)\left(\bar{\nu} \gamma_{\mu} \gamma_{5} \ell\right) \\
& +\frac{m_{\ell}}{M_{W}} c_{S}(\bar{s} u)(\bar{\nu} \ell)+i \frac{m_{\ell}}{M_{W}} c_{P}(\bar{s} u)\left(\bar{\nu} \gamma_{5} \ell\right) \\
& +\frac{m_{s} m_{\ell}}{M_{W}^{2}} c^{T}\left(\bar{s} \sigma^{\mu \nu} u\right)\left(\bar{\nu} \sigma^{\mu \nu} \ell\right)+\frac{m_{s} m_{\ell}}{M_{W}^{2}} c_{\gamma_{5}}^{T}\left(\bar{s} \sigma^{\mu \nu} u\right)\left(\bar{\nu} \sigma^{\mu \nu} \gamma_{5} \ell\right)+\text { h.c. }|K\rangle
\end{aligned}
$$

where

$$
\begin{aligned}
& c_{V}=+\left(c_{L L}^{V}+c_{R L}^{V}+c_{L R}^{V}+c_{R R}^{V}\right), \\
& c_{A}=-\left(c_{L L}^{V}+c_{R L}^{V}-c_{L R}^{V}-c_{R R}^{V}\right), \\
& c_{S}=+\left(c_{L L}^{S}+c_{R L}^{S}+c_{L R}^{S}+c_{R R}^{S}\right) M_{W} / m_{\ell},
\end{aligned}
$$




$$
\begin{aligned}
i c_{P} & =-\left(c_{L L}^{S}+c_{R L}^{S}-c_{L R}^{S}-c_{R R}^{S}\right) M_{W} / m_{\ell}, \\
c^{T} & =2\left(c_{L L}^{T}+c_{R R}^{T}\right) M_{W}^{2} /\left(m_{\ell} m_{s}\right), \quad c_{\gamma_{5}}^{T}=-2\left(c_{L L}^{T}-c_{R R}^{T}\right) M_{W}^{2} /\left(m_{\ell} m_{s}\right) .
\end{aligned}
$$

Similarly, in the $K \rightarrow \ell \nu$ case the independent structures are

$$
\begin{aligned}
\mathcal{A}(K \rightarrow \ell \nu) & =-\frac{G_{F}}{\sqrt{2}} V_{u s}\langle\ell \nu| k_{V}\left(\bar{s} \gamma^{\mu} \gamma_{5} u\right)\left(\bar{\nu} \gamma_{\mu} \ell\right)+k_{A}\left(\bar{s} \gamma^{\mu} \gamma_{5} u\right)\left(\bar{\nu} \gamma_{\mu} \gamma_{5} \ell\right) \\
& +\frac{m_{\ell}}{M_{W}} k_{S}\left(\bar{s} \gamma_{5} u\right)(\bar{\nu} \ell)+\frac{m_{\ell}}{M_{W}} k_{P}\left(\bar{s} \gamma_{5} u\right)\left(\bar{\nu} \gamma_{5} \ell\right)+\text { h.c. }|K\rangle
\end{aligned}
$$

where

$$
\begin{aligned}
& k_{V}=-\left(c_{L L}^{V}-c_{R L}^{V}+c_{L R}^{V}-c_{R R}^{V}\right), \\
& k_{A}=+\left(c_{L L}^{V}-c_{R L}^{V}-c_{L R}^{V}+c_{R R}^{V}\right), \\
& k_{S}=-\left(c_{L L}^{S}-c_{R L}^{S}+c_{L R}^{S}-c_{R R}^{S}\right) M_{W} / m_{\ell}, \\
& k_{P}=+\left(c_{L L}^{S}-c_{R L}^{S}-c_{L R}^{S}+c_{R R}^{S}\right) M_{W} / m_{\ell} .
\end{aligned}
$$

On general grounds, new degrees of freedom weakly coupled at the scale $\Lambda_{\mathrm{NP}}$ are expected to generate corrections of $\mathcal{O}\left(M_{W}^{2} / \Lambda_{\mathrm{NP}}^{2}\right)$ to the Wilson coefficients of $H_{s u}^{\Delta S=1}$. Focusing on well-motivated new-physics frameworks, the following two scenarios are particularly interesting:

- In two Higgs doublet models of type-II, such as the Higgs sector of the MSSM, sizable contributions are potentially generated by charged-Higgs exchange diagrams (see e.g. Ref. [24, 25, 26]). These are well described by the following set of initial conditions for $s \rightarrow u$ transitions,

$$
c_{L L}^{V}=1 \quad \text { and } \quad c_{L R}^{S}=-\frac{\tan ^{2} \beta}{\left(1+\epsilon_{0} \tan \beta\right)} \frac{m_{\ell} m_{s}}{m_{H^{+}}^{2}},
$$

and for $u \rightarrow d$ transitions,

$$
c_{L L}^{V, u d}=1 \quad \text { and } \quad c_{L R}^{S, u d}=-\frac{\tan ^{2} \beta}{\left(1+\epsilon_{0} \tan \beta\right)} \frac{m_{\ell} m_{d}}{m_{H^{+}}^{2}} .
$$

Here $\tan \beta$ is the ratio of the two Higgs vacuum expectation values and $\epsilon_{0}$ is a loop function whose detailed expression can be found in Ref. [25]. In presence of sizable sources of lepton-flavor symmetry breaking, a non-vanishing scalar-current contribution to the lepton-flavor violating process $K \rightarrow e \nu_{\tau}$ is also present [26]. The latter can be parametrized by

$$
c_{L R}^{S^{\prime}}=\frac{m_{s} m_{\tau}}{m_{H^{+}}^{2}} \Delta_{R}^{31} \tan ^{2} \beta .
$$

- In the Higgs-less model of Ref. [12], non-standard right-handed quark currents could become detectable. These are described by the following set of initial conditions for both $u \rightarrow s$ and $u \rightarrow d$ transitions

$$
\begin{aligned}
& c_{L L}^{V}=(1+\delta) \quad \text { and } \quad c_{R L}^{V}=\epsilon_{s}, \\
& c_{L L}^{V, u d}=(1+\delta) \quad \text { and } \quad c_{R L}^{V, u d}=\epsilon_{n s},
\end{aligned}
$$

where $\varepsilon_{x}$ and $\delta$ are free parameters of the model. $\epsilon_{s}$ can reach a few percents if the hierarchy of the right-handed mixing matrix is inverted. 


\subsection{2 $K_{\ell 2}$ rates}

According to the Hamiltonian of Eq. (2.27), the $K_{\ell 2}$ rate of Eq. (2.2) can be modified as

$$
\begin{aligned}
\frac{\Gamma\left(K_{\ell 2(\gamma)}^{ \pm}\right)}{\Gamma\left(\pi_{\ell 2(\gamma)}^{ \pm}\right)}=\left|\frac{V_{u s}}{V_{u d}}\right|^{2} & \frac{f_{K}^{2} m_{K}}{f_{\pi}^{2} m_{\pi}}\left(\frac{1-m_{\ell}^{2} / m_{K}^{2}}{1-m_{\ell}^{2} / m_{\pi}^{2}}\right)^{2} \times\left(1+\delta_{\mathrm{em}}\right) \\
& \times \frac{\left|k_{A}-m_{K}^{2} /\left(m_{s} M_{W}\right) k_{P}\right|^{2}+\left|k_{V}+m_{K}^{2} /\left(m_{s} M_{W}\right) k_{S}\right|^{2}}{\left|k_{A}^{u d}-m_{\pi}^{2} /\left(\hat{m} M_{W}\right) k_{P}^{u d}\right|^{2}+\left|k_{V}^{u d}+m_{\pi}^{2} /\left(\hat{m} M_{W}\right) k_{S}^{u d}\right|^{2}}
\end{aligned}
$$

where $\hat{m}=m_{u}+m_{d}$ and $k_{x}^{u d}$ are defined for the $u \rightarrow d$ transition. In the MSSM scenario

$$
\Gamma^{\operatorname{MSSM}}\left(K_{\ell 2}\right) / \Gamma^{\mathrm{MSSM}}\left(\pi_{\ell 2}\right)=\Gamma^{\mathrm{SM}}\left(K_{\ell 2}\right) / \Gamma^{\mathrm{SM}}\left(\pi_{\ell 2}\right) \times\left(1-r_{H}^{K}\right)^{2}
$$

where

$$
r_{H}^{K}=\frac{m_{K^{+}}^{2}}{M_{H^{+}}^{2}}\left(1-\frac{m_{d}}{m_{s}}\right) \frac{\tan ^{2} \beta}{1+\epsilon_{0} \tan \beta}
$$

\subsection{3 $K_{\ell 3}$ rates and kinematical distributions}

In the $K_{\ell 3}$ case the non-standard operators of Eq. (2.21) could in principle modify the Daliz plot distribution. However, as we will show in the following, this effect turns out to be hardly detectable for most realistic new-physics scenarios.

The hadronic form factors needed in the general case are the two FFs defined in Eq. (2.4) plus a tensor FF, whereas $f_{0}(t)$ allow us to parametrize also the scalar-current matrix element. More specifically, we have

$$
\begin{aligned}
\left\langle\pi^{-}(k)|(\bar{s} u)| K^{0}(p)\right\rangle & =-\frac{m_{K}^{2}-m_{\pi}^{2}}{\left(m_{s}-m_{u}\right)} f_{0}(t), \\
\left\langle\pi^{-}(k)\left|\left(\bar{s} \sigma^{\mu \nu} u\right)\right| K^{0}(p)\right\rangle & =i \frac{p^{\mu} k^{\nu}-p^{\nu} k^{\mu}}{m_{K}} B_{T}(t) .
\end{aligned}
$$

The tensor form-factor was studied on the lattice [13], with the result $B_{T}(t) \approx 1.2(1) f_{+}(0) /(1-$ $0.3(1) t$ ) at $\mu \simeq 2 \mathrm{GeV}$ in the $\overline{M S}$ scheme (an earlier order-of-magnitude estimate may be found in Ref. [14]).

Choosing as independent kinematical variables

$$
z=\frac{2 p_{K} \cdot p_{\pi}}{m_{K}^{2}}=\frac{m_{K}^{2}+m_{\pi}^{2}-t}{m_{K}^{2}}, \quad y=\frac{2 p_{K} \cdot p_{\ell}}{m_{K}^{2}}, \quad r_{\pi, \ell}=\frac{m_{\pi, \ell}^{2}}{m_{K}^{2}}
$$

the double differential density can be written as (neglecting long-distance electromagnetic corrections)

$$
\begin{aligned}
\frac{d \Gamma}{d y d z} & =\frac{G_{F}^{2}\left|V_{u s}\right|^{2} m_{K}^{5}}{256 \pi^{3}} C_{K} S_{\text {ew }}\left[A_{1}(y, z)\left(|V|^{2}+|A|^{2}\right)-A_{2}(y, z) \operatorname{Re}\left(V S^{*}-A P^{*}\right)\right. \\
& \left.+A_{3}(y, z)\left(|S|^{2}+|P|^{2}\right)\right]
\end{aligned}
$$


whereas

$$
\begin{aligned}
& A_{1}(y, z)=4(z+y-1)(1-y)+r_{\ell}(4 y+3 z-3)-4 r_{\pi}+r_{\ell}\left(r_{\pi}-r_{\ell}\right), \\
& A_{2}(y, z)=2 r_{\ell}\left(3-2 y-z+r_{\ell}-r_{\pi}\right), \quad A_{3}(y, z)=r_{\ell}\left(1+r_{\pi}-z-r_{\ell}\right) .
\end{aligned}
$$

Here $S, P, V$, and $A$ are convenient combinations of hadronic form factors and shortdistance Wilson coefficients:

$$
\begin{aligned}
& V(t, y)=f_{+}(t) c_{V}-m_{\ell}^{2} \frac{m_{s}}{M_{W}^{2}} \frac{c_{T} B_{T}(t)}{m_{K}} \\
& A(t, y)=f_{+}(t) c_{A}+m_{\ell}^{2} \frac{m_{s}}{M_{W}^{2}} \frac{c_{T 5} B_{T}(t)}{m_{K}} \\
& S(t, y)=-\left(f_{0}^{S}(t)-f_{+}(t)\right) \frac{m_{K}^{2}-m_{\pi}^{2}}{t} c_{V}-\left(m_{\ell}^{2}+m_{K}^{2}(2-z-2 y)\right) \frac{m_{s}}{M_{W}^{2}} \frac{c_{T} B_{T}(t)}{m_{K}} \\
& P(t, y)=\left(f_{0}^{P}(t)-f_{+}(t)\right) \frac{m_{K}^{2}-m_{\pi}^{2}}{t} c_{A}-\left(m_{\ell}^{2}+m_{K}^{2}(2-z-2 y)\right) \frac{m_{s}}{M_{W}^{2}} \frac{c_{T 5} B_{T}(t)}{m_{K}}
\end{aligned}
$$

where

$$
\begin{aligned}
& f_{0}^{S}(t)=f_{0}(t)\left(1+\frac{c_{S} / c_{V}}{\left(m_{s}-m_{u}\right) M_{W}} t\right) \approx f_{0}(t) \exp \left(\frac{c_{S} / c_{V}}{M_{W}} \frac{m_{K}^{2}-m_{\pi}^{2}}{m_{s}-m_{u}}\right)^{t / t_{C T}} \\
& f_{0}^{P}(t)=f_{0}(t)\left(1-\frac{i c_{P} / c_{A}}{\left(m_{s}-m_{u}\right) M_{W}} t\right) \approx f_{0}(t) \exp \left(\frac{-i c_{P} / c_{A}}{M_{W}} \frac{m_{K}^{2}-m_{\pi}^{2}}{m_{s}-m_{u}}\right)^{t / t_{C T}}
\end{aligned}
$$

$t_{C T}=\left(m_{K}^{2}-m_{\pi}^{2}\right)$ and we have assumed $c_{S, P} / c_{V, A} \ll 1$. The SM case is recovered from Eq. (2.44) in the limit $c_{V}=-c_{A}=1$ and $f_{0}^{S, P}(t)=f_{0}(t)$.

After integrating over $y$, differences to the SM rate of Eq. (2.1) can be summarized as it follows. Right-handed currents can only rescale the overall rate of Eq. (2.1), namely

$$
\Gamma\left(K_{\ell 3(\gamma)}\right) \rightarrow \Gamma\left(K_{\ell 3(\gamma)}\right) \times \frac{\left|c_{V}\right|^{2}+\left|c_{A}\right|^{2}}{2} .
$$

Scalar and pseudoscalar contributions can be easily encoded in Eq. (2.1) by substituting

$$
f_{0}(t) \rightarrow f_{0}^{H}(t)=f_{0}(t) \exp \left(\frac{\left(-i c_{P} c_{A}^{*}+c_{S} c_{V}^{*}\right)}{\left|c_{V}\right|^{2}+\left|c_{A}\right|^{2}} \frac{m_{K}^{2}-m_{\pi}^{2}}{M_{W} m_{s}}\right)^{t / t_{C T}} .
$$

In particular, these new effects are vanishing for $t=0$, namely $f_{0}(0)$ in Eq. (2.1) is free from them. The tensor coupling modify the phase space integral $I_{K}^{\ell}\left(\lambda_{+, 0}\right)$ of Eq. (2.1) by

$$
I_{K}^{\ell}\left(\lambda_{+, 0}\right) \rightarrow I_{K}^{\ell}\left(\lambda_{+, 0}\right)-\frac{\operatorname{Re}\left(c^{T} c_{V}^{*}\right)-\operatorname{Re}\left(c_{\gamma_{5}}^{T} c_{A}^{*}\right)}{\left|c_{V}\right|^{2}+\left|c_{A}\right|^{2}} I_{T}^{\ell}\left(\lambda_{T,+, 0}\right)
$$

In conclusion, the integrated rate including electromagnetic corrections can be written as

$$
\begin{aligned}
\Gamma\left(K_{\ell 3(\gamma)}\right) & =\frac{G_{F}^{2} m_{K}^{5}}{192 \pi^{3}} C_{K} S_{\mathrm{ew}}\left|V_{u s}\right|^{2} f_{+}(0)^{2}\left(1+\delta_{S U(2)}^{K}+\delta_{\mathrm{em}}^{K \ell}\right)^{2} \\
& \times \frac{\left|c_{V}\right|^{2}+\left|c_{A}\right|^{2}}{2}\left(I_{K}^{\ell}-\frac{\operatorname{Re}\left(c^{T} c_{V}^{*}\right)-\operatorname{Re}\left(c_{\gamma_{5}}^{T} c_{A}^{*}\right)}{\left|c_{V}\right|^{2}+\left|c_{A}\right|^{2}} I_{T}^{\ell}\right)
\end{aligned}
$$


where

$$
\begin{gathered}
I_{K}^{\ell}=\frac{1}{m_{K}^{2} f_{+}(0)^{2}} \int d t \lambda^{3 / 2}(t)\left(1+\frac{m_{\ell}^{2}}{2 t}\right)\left(1-\frac{m_{\ell}^{2}}{t}\right)^{2} \\
\times\left(f_{+}^{2}(t)+\frac{3 m_{\ell}^{2}\left(m_{K}^{2}-m_{\pi}^{2}\right)^{2}}{\left(2 t+m_{\ell}^{2}\right) m_{K}^{4} \lambda(t)}\left|f_{0}^{H}(t)\right|^{2}\right), \\
I_{T}^{\ell}=\frac{1}{m_{K}^{2} f_{+}(0)^{2}} \int d t \lambda^{3 / 2}(t) \frac{m_{\ell}}{4 m_{K}}\left(1+\frac{2 m_{\ell}^{2}}{t}\right)\left(1-\frac{m_{\ell}^{2}}{t}\right)^{2} \\
B_{T}(t) \times\left(f_{+}(t)+6 \frac{2 m_{\ell}^{2}\left(m_{K}^{2}-m_{\pi}^{2}\right)^{2}-\left(m_{K}^{4}-m_{\pi}^{4}\right) t+t^{2}}{\left(t+2 m_{\ell}^{2}\right) m_{K}^{4} \lambda(t)} f_{0}(t)\right)
\end{gathered}
$$

and $\lambda(t)=1-2 r_{\pi}^{2}+r_{\pi}^{4}-2 t / m_{K}^{2}-2 r_{\pi}^{2} t / m_{K}^{2}+t^{2} / m_{K}^{4}$.

In most realistic new-physics scenarios the modification of the $K_{\ell 3}$ scalar form factor is well below the present experimental and theoretical errors. For instance, in the MSSM (or two-Higgs doublets) case $f_{0}^{H}(t)$ reads

$$
\left.f_{0}^{H}(t)\right|_{\mathrm{MSSM}}=f_{0}(t) \exp \left(-r_{H}^{K}\right)^{t / t_{C T}}
$$

where $r_{H}^{K}$ is the parameter controlling the corrections to the $K_{\ell 2}$ rate of Eq. (2.38). For natural values of the free parameters $\left(\epsilon_{0}=10^{-2}, M_{H^{+}}^{2}=400 \mathrm{GeV}\right.$ and $\left.\tan \beta=40\right)$, such that $r_{H}^{K}=0.2 \%$, the corresponding modification of the $K_{\ell 3}$ scalar form factor is

$$
\frac{\delta \lambda_{0}}{\lambda_{0}^{\mathrm{SM}}} \approx 1.0 \% \quad \text { or } \quad \frac{\delta f_{0}\left(t_{C T}\right)}{f_{0}\left(t_{C T}\right)^{\mathrm{SM}}} \approx 0.18 \%
$$

well below the level of present theoretical and experimental uncertainties.

\section{Data Analysis}

We perform fits to world data on the BRs and lifetimes for the $K_{L}$ and $K^{ \pm}$, with the constraint that BRs add to unity. This is the correct way of using the new measurements. The fit procedure is described in Appendix A.

\section{1 $K_{L}$ leading branching ratios and $\tau_{L}$}

Numerous measurements of the principal $K_{L}$ BRs, or of various ratios of these BRs, have been published recently. For the purposes of evaluating $\left|V_{u s}\right| f_{+}(0)$, these data can be used in a PDG-like fit to the $K_{L}$ BRs and lifetime, so all such measurements are interesting. A detailed description to the fit of the principal $K_{L}$ BRs and $\tau_{L}$ is given in Appendix B

$\mathrm{KTeV}$ has measured five ratios of the six main $K_{L}$ BRs [27]. The six channels involved account for more than $99.9 \%$ of the $K_{L}$ width and $\mathrm{KTeV}$ combines the five measured ratios to extract the six BRs. We use the five measured ratios in our analysis: $\mathrm{BR}\left(K_{\mu 3}\right) / \mathrm{BR}\left(K_{e 3}\right)=0.6640(26), \mathrm{BR}\left(\pi^{+} \pi^{-} \pi^{0}\right) / \mathrm{BR}\left(K_{e 3}\right)=0.3078(18), \mathrm{BR}\left(\pi^{+} \pi^{-}\right) / \mathrm{BR}\left(K_{e 3}\right)=$ $0.004856(28), \operatorname{BR}\left(3 \pi^{0}\right) / \operatorname{BR}\left(K_{e 3}\right)=0.4782(55)$, and $\operatorname{BR}\left(2 \pi^{0}\right) / \operatorname{BR}\left(3 \pi^{0}\right)=0.004446(25)$.

The errors on these measurements are correlated; this is taken into account in our fit. 


\begin{tabular}{l|c|c} 
Parameter & Value & $S$ \\
\hline $\operatorname{BR}\left(K_{e 3}\right)$ & $0.4056(7)$ & 1.1 \\
$\operatorname{BR}\left(K_{\mu 3}\right)$ & $0.2705(7)$ & 1.1 \\
$\operatorname{BR}\left(3 \pi^{0}\right)$ & $0.1951(9)$ & 1.2 \\
$\operatorname{BR}\left(\pi^{+} \pi^{-} \pi^{0}\right)$ & $0.1254(6)$ & 1.1 \\
$\operatorname{BR}\left(\pi^{+} \pi^{-}\right)$ & $1.997(7) \times 10^{-3}$ & 1.1 \\
$\operatorname{BR}\left(2 \pi^{0}\right)$ & $8.64(4) \times 10^{-4}$ & 1.3 \\
$\operatorname{BR}(\gamma \gamma)$ & $5.47(4) \times 10^{-4}$ & 1.1 \\
$\tau_{L}$ & $51.17(20) \mathrm{ns}$ & 1.1
\end{tabular}

Table 2: Results of fit to $K_{L}$ BRs and lifetime.

NA48 has measured the ratio of the BR for $K_{e 3}$ decays to the sum of BRs for all decays to two tracks, giving $\mathrm{BR}\left(K_{e 3}\right) /\left(1-\mathrm{BR}\left(3 \pi^{0}\right)\right)=0.4978(35)$ [28]. From a separate measurement of $\operatorname{BR}\left(K_{L} \rightarrow 3 \pi^{0}\right) / \operatorname{BR}\left(K_{S} \rightarrow 2 \pi^{0}\right)$, NA48 obtains $\operatorname{BR}\left(3 \pi^{0}\right) / \tau_{L}=3.795(58)$ $\mu \mathrm{s}^{-1}$ [29].

Using $\phi \rightarrow K_{L} K_{S}$ decays in which the $K_{S}$ decays to $\pi^{+} \pi^{-}$, providing normalization, KLOE has directly measured the BRs for the four main $K_{L}$ decay channels [30]. The errors on the KLOE BR values are dominated by the uncertainty on the $K_{L}$ lifetime $\tau_{L}$; since the dependence of the geometrical efficiency on $\tau_{L}$ is known, KLOE can solve for $\tau_{L}$ by imposing $\sum_{x} \mathrm{BR}\left(K_{L} \rightarrow x\right)=1$ (using previous averages for the minor BRs), thereby greatly reducing the uncertainties on the BR values obtained. Our fit makes use of the KLOE BR values before application of this constraint: $\operatorname{BR}\left(K_{e 3}\right)=0.4049(21), \operatorname{BR}\left(K_{\mu 3}\right)=$ $0.2726(16), \operatorname{BR}\left(K_{e 3}\right)=0.2018(24)$, and $\mathrm{BR}\left(K_{e 3}\right)=0.1276(15)$. The dependence of these values on $\tau_{L}$ and the correlations between the errors are taken into account. KLOE has also measured $\tau_{L}$ directly, by fitting the proper decay time distribution for $K_{L} \rightarrow 3 \pi^{0}$ events, for which the reconstruction efficiency is high and uniform over a fiducial volume of $\sim 0.4 \lambda_{L}$. They obtain $\tau_{L}=50.92(30)$ ns [31].

There are also two recent measurements of $\mathrm{BR}\left(\pi^{+} \pi^{-}\right) / \mathrm{BR}\left(K_{\ell 3}\right)$, in addition to the $\mathrm{KTeV}$ measurement of $\mathrm{BR}\left(\pi^{+} \pi^{-}\right) / \mathrm{BR}\left(K_{e 3}\right)$ discussed above. The KLOE collaboration obtains $\operatorname{BR}\left(\pi^{+} \pi^{-}\right) / \mathrm{BR}\left(K_{\mu 3}\right)=7.275(68) \times 10^{-3}$ 32, while NA48 obtains $\operatorname{BR}\left(\pi^{+} \pi^{-}\right) / \operatorname{BR}\left(K_{e 3}\right)$ $=4.826(27) \times 10^{-3}$ [33. All measurements are fully inclusive of inner bremsstrahlung. The KLOE measurement is fully inclusive of the direct-emission (DE) component, DE contributes negligibly to the $\mathrm{KTeV}$ measurement, and a residual DE contribution of $0.19 \%$ has been subtracted from the NA48 value to obtain the number quoted above. For consistency, in our fit, a DE contribution of $1.52(7) \%$ is added to the $\mathrm{KTeV}$ and NA48 values. Our fit result for $\mathrm{BR}\left(\pi^{+} \pi^{-}\right)$is then understood to be DE inclusive.

In addition to the 14 recent measurements listed above, our fit for the seven largest $K_{L}$ BRs and lifetime uses four of the remaining five inputs to the 2006 PDG fit and the constraint that the seven BRs add to unity. The results are given in Table 2 .

The evolution of the average values of the BRs for $K_{L \ell 3}$ decays and for the important normalization channels is shown in Fig. 2. 


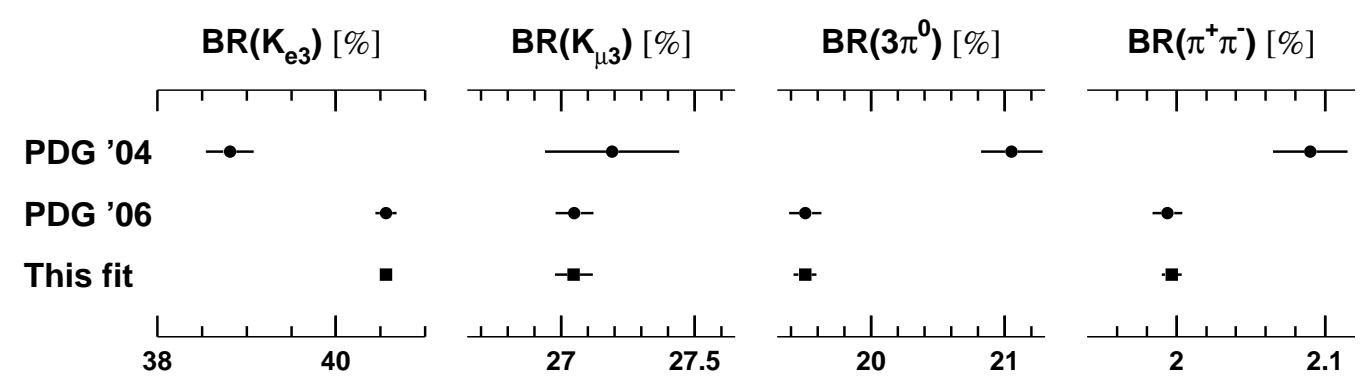

Figure 1: Evolution of average values for main $K_{L}$ BRs.

Our fit gives $\chi^{2} / \mathrm{ndf}=20.2 / 11(4.3 \%)$, while the $2006 \mathrm{PDG}$ fit gives $\chi^{2} / \mathrm{ndf}=14.8 / 10$ (14.0\%). The differences between the output values from our fit and the 2006 PDG fit are minor. The poorer value of $\chi^{2} /$ ndf for our fit can be traced to contrast between the KLOE value for $\operatorname{BR}\left(3 \pi^{0}\right)$ and the other inputs involving $\operatorname{BR}\left(3 \pi^{0}\right)$ and $\operatorname{BR}\left(\pi^{0} \pi^{0}\right)$ - in particular, the PDG ETAFIT value for $\mathrm{BR}\left(\pi^{0} \pi^{0} / \pi^{+} \pi^{-}\right)$. The treatment of the correlated $\mathrm{KTeV}$ and KLOE measurements in the 2006 PDG fit gives rise to large scale factors for $\operatorname{BR}\left(K_{e 3}\right)$ and $\operatorname{BR}\left(3 \pi^{0}\right)$; in our fit, the scale factors are more uniform. As a result, our value for $\operatorname{BR}\left(K_{e 3}\right)$ has a significantly smaller uncertainty than does the 2006 PDG value.

\section{2 $K_{S}$ leading branching ratios and $\tau_{S}$}

KLOE has published [34] a measurement of $\mathrm{BR}\left(K_{S} \rightarrow \pi e \nu\right)$ that is precise enough to contribute meaningfully to the evaluation of $\left|V_{u s}\right| f_{+}(0)$. The quantity directly measured is $\operatorname{BR}(\pi e \nu) / \operatorname{BR}\left(\pi^{+} \pi^{-}\right)$. Together with the published $\operatorname{KLOE}$ value $\operatorname{BR}\left(\pi^{+} \pi^{-}\right) / \operatorname{BR}\left(\pi^{0} \pi^{0}\right)$ $=2.2459(54)$, the constraint that the $K_{S}$ BRs must add to unity, and the assumption of universal lepton couplings, this completely determines the $K_{S}$ BRs for $\pi^{+} \pi^{-}, \pi^{0} \pi^{0}, K_{e 3}$, and $K_{\mu 3}$ decays [35]. In particular, $\operatorname{BR}\left(K_{S} \rightarrow \pi e \nu\right)=7.046(91) \times 10^{-4}$.

NA48 has recently measured the ratio $\Gamma\left(K_{S} \rightarrow \pi e \nu\right) / \Gamma\left(K_{L} \rightarrow \pi e \nu\right)=0.993(26)(22)$ [36]. The best way to include this measurement in our analysis would be via a combined fit to $K_{S}$ and $K_{L}$ branching ratio and lifetime measurements. Indeed, such a fit would be useful in properly accounting for correlations between $K_{S}$ and $K_{L}$ modes introduced with the preliminary NA48 measurement of $\Gamma\left(K_{L} \rightarrow 3 \pi^{0}\right)$, and more importantly, via the PDG ETAFIT result, which we use in the fit to $K_{L}$ branching ratios. At the moment, however, we fit $K_{S}$ and $K_{L}$ data separately. NA48 quotes $\operatorname{BR}\left(K_{S} \rightarrow \pi e \nu\right)=7.046(180)(160) \times 10^{-4}$; averaging this with the KLOE result gives $\mathrm{BR}\left(K_{S} \rightarrow \pi e \nu\right)=7.05(8) \times 10^{-4}$, improving the accuracy on this BR by about $10 \%$.

For $\tau_{K_{S}}$ we use $0.8958 \times 10^{-10} \mathrm{~s}$, where this is the non- $C P T$ constrained fit value from the PDG, and is dominated by the 2002 NA48 and $2003 \mathrm{KTeV}$ measurements.

\section{3 $K^{ \pm}$leading branching ratios and $\tau^{ \pm}$}

There are several new results providing information on $K_{\ell 3}^{ \pm}$rates. These results are mostly preliminary and have not been included in previous averages. 


\begin{tabular}{l|c|c} 
Parameter & Value & $S$ \\
\hline $\operatorname{BR}\left(K_{\mu 2}\right)$ & $63.57(11) \%$ & 1.1 \\
$\operatorname{BR}\left(\pi \pi^{0}\right)$ & $20.64(8) \%$ & 1.1 \\
$\operatorname{BR}(\pi \pi \pi)$ & $5.595(31) \%$ & 1.0 \\
$\operatorname{BR}\left(K_{e 3}\right)$ & $5.078(26) \%$ & 1.2 \\
$\operatorname{BR}\left(K_{\mu 3}\right)$ & $3.365(27) \%$ & 1.7 \\
$\operatorname{BR}\left(\pi \pi^{0} \pi^{0}\right)$ & $1.750(26) \%$ & 1.1 \\
$\tau_{ \pm}$ & $12.384(19) \mathrm{ns}$ & 1.7
\end{tabular}

Table 3: Results of fit to $K^{ \pm}$BRs and lifetime.

The NA48/2 collaboration has recently published measurements of the three ratios $\mathrm{BR}\left(K_{e 3} / \pi \pi^{0}\right), \operatorname{BR}\left(K_{\mu 3} / \pi \pi^{0}\right)$, and $\operatorname{BR}\left(K_{\mu 3} / K_{e 3}\right)$ [37]. These measurements are not independent; in our fit, we use the values $\operatorname{BR}\left(K_{e 3} / \pi \pi^{0}\right)=0.2470(10)$ and $\operatorname{BR}\left(K_{\mu 3} / \pi \pi^{0}\right)=$ $0.1637(7)$ and take their correlation into account. ISTRA+ has also updated its preliminary value for $\operatorname{BR}\left(K_{e 3} / \pi \pi^{0}\right)$. They now quote $\operatorname{BR}\left(K_{e 3} / \pi \pi^{0}\right)=0.2449(16)$ [38].

KLOE has measured the absolute BRs for the $K_{e 3}$ and $K_{\mu 3}$ decays [39]. In $\phi \rightarrow K^{+} K^{-}$ events, $K^{+}$decays into $\mu \nu$ or $\pi \pi^{0}$ are used to tag a $K^{-}$beam, and vice versa. KLOE performs four separate measurements for each $K_{\ell 3} \mathrm{BR}$, corresponding to the different combinations of kaon charge and tagging decay. The final averages are $\operatorname{BR}\left(K_{e 3}\right)=4.965(53) \%$ and $\operatorname{BR}\left(K_{\mu 3}\right)=3.233(39) \%$. Very recently KLOE has also measured the absolute branching ratio for the $\pi \pi^{0}$ decay with $0.5 \%$ accuracy. The KLOE preliminary result, is $\operatorname{BR}\left(\pi \pi^{0}\right)=$ $0.20658(112) 41$.

Our fit takes into account the correlation between these values, as well as their dependence on the $K^{ \pm}$lifetime. The world average value for $\tau_{ \pm}$is nominally quite precise; the 2006 PDG quotes $\tau_{ \pm}=12.385(25)$ ns. However, the error is scaled by 2.1 ; the confidence level for the average is $0.17 \%$. It is important to confirm the value of $\tau_{ \pm}$. The two new measurements from KLOE, $\tau_{ \pm}=12.367(44)(65)$ ns and $\tau_{ \pm}=12.391(49)(25)$ ns [42] with correlation $34 \%$, agree with the PDG average.

Our fit for the six largest $K^{ \pm}$BRs and lifetime makes use of the results cited above, plus the data used in the 2006 PDG fit, except for the Chiang '72 measurements for a total of 26 measurements. The six BRs are constrained to add to unity. The results are shown in Table 3 .

The fit quality is poor, with $\chi^{2} / \mathrm{ndf}=42 / 20$ (0.31\%). However, when the five older measurements of $\tau_{ \pm}$are replaced by their PDG average with scaled error, $\chi^{2} /$ ndf improves to $24.3 / 16(8.4 \%)$, with no significant changes in the results.

Both the significant evolution of the average values of the $K_{\ell 3}$ BRs and the effect of the correlations with $\operatorname{BR}\left(\pi \pi^{0}\right)$ are evident in Fig. 2 .

\subsection{Measurement of $\mathbf{B R}\left(K_{e 2}\right) / \mathbf{B R}\left(K_{\mu 2}\right)$}

Experimental knowledge of $K_{e 2} / K_{\mu 2}$ has been poor so far. The current world average of $R_{K}=\mathrm{BR}\left(K_{e 2}\right) / \mathrm{BR}\left(K_{\mu 2}\right)=(2.45 \pm 0.11) \times 10^{-5}$ dates back to three experiments of the 

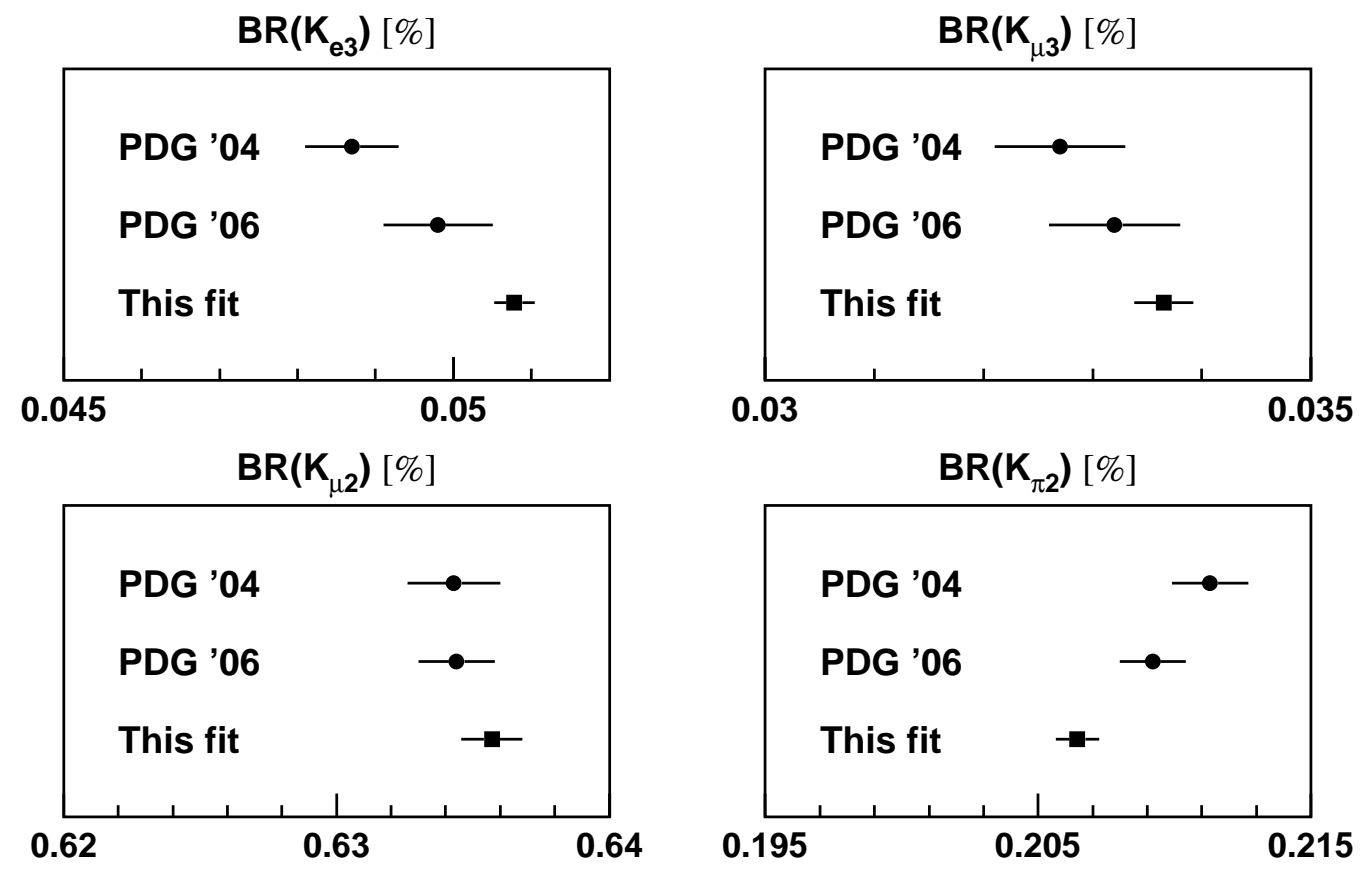

Figure 2: Evolution of average values for main $K^{ \pm}$BRs.

1970s 443 and has a precision of about 5\%. Three new preliminary measurements were reported by NA48/2 and KLOE (see Tab. 国): A preliminary result of NA48/2, based on about $4000 K_{e 2}$ events from the 2003 data set, was presented in 2005 [44. Another preliminary result, based on also about 4000 events, recorded in a minimum bias run period in 2004, was shown at KAON07 [45]. Both results have independent statistics and are also independent in the systematic uncertainties, as the systematics are either of statistical nature (as e.g. trigger efficiencies) or determined in an independent way. Another preliminary result, based on about $8000 K_{e 2}$ events, was presented at KAON07 by the KLOE collaboration [46]. Both, the KLOE and the NA48/2 measurements are inclusive with respect to final state radiation contribution due to bremsstrahlung. The small contribution of $K_{l 2 \gamma}$ events from direct photon emission from the decay vertex was subtracted by each of the experiments. Combining these new results with the current PDG value yields a current world average of

$$
R_{K}=(2.457 \pm 0.032) \times 10^{-5}
$$

in very good agreement with the SM expectation and, with a relative error of $1.3 \%$, a factor three more precise than the previous world average.

\subsection{Measurements of $K_{\ell 3}$ slopes}

\subsubsection{Vector form factor slopes from $K_{\ell 3}$}

For $K_{e 3}$ decays, recent measurements of the quadratic slope parameters of the vector form factor $\left(\lambda_{+}^{\prime}, \lambda_{+}^{\prime \prime}\right)$ are available from $\mathrm{KTeV}$ [48], KLOE [49], ISTRA+ [50], and NA48 [51]. 


\begin{tabular}{lc}
\hline \hline & $R_{K}\left[10^{-5}\right]$ \\
\hline PDG 2006 [43] & $2.45 \pm 0.11$ \\
NA48/2 prel. ('03) 444 & $2.416 \pm 0.043 \pm 0.024$ \\
NA48/2 prel. ('04) 45] & $2.455 \pm 0.045 \pm 0.041$ \\
KLOE prel. [46] & $2.55 \pm 0.05 \pm 0.05$ \\
\hline SM prediction & $2.477 \pm 0.001$ \\
\hline \hline
\end{tabular}

Table 4: Results and prediction for $R_{K}=\mathrm{BR}\left(K_{e 2}\right) / \mathrm{BR}\left(K_{\mu 2}\right)$.

We show the results of a fit to the $K_{L}$ and $K^{-}$data in the first column of Table 5 , and to only the $K_{L}$ data in the second column. With correlations correctly taken into account, both fits give good values of $\chi^{2} /$ ndf. The significance of the quadratic term is $4.2 \sigma$ from the fit to all data, and $3.5 \sigma$ from the fit to $K_{L}$ data only.

\begin{tabular}{lcc}
\hline \hline & $K_{L}$ and $K^{-}$data & $K_{L}$ data only \\
& 4 measurements & 3 measurements \\
& $\chi^{2} / \mathrm{ndf}=5.3 / 6(51 \%)$ & $\chi^{2} / \mathrm{ndf}=4.7 / 4(32 \%)$ \\
\hline$\lambda_{+}^{\prime} \times 10^{3}$ & $25.2 \pm 0.9$ & $24.9 \pm 1.1$ \\
$\lambda_{+}^{\prime \prime} \times 10^{3}$ & $1.6 \pm 0.4$ & $1.6 \pm 0.5$ \\
$\rho\left(\lambda_{+}^{\prime}, \lambda+{ }^{\prime \prime}\right)$ & -0.94 & -0.95 \\
$I\left(K_{e 3}^{0}\right)$ & $0.15465(24)$ & $0.15456(31)$ \\
$I\left(K_{e 3}^{ \pm}\right)$ & $0.15901(24)$ & $0.15891(32)$ \\
\hline \hline
\end{tabular}

Table 5: Average of quadratic fit results for $K_{e 3}$ slopes.

Including or excluding the $K^{-}$slopes has little impact on the values of $\lambda_{+}^{\prime}$ and $\lambda_{+}^{\prime \prime}$; in particular, the values of the phase-space integrals change by just $0.07 \%$. The errors on the phase-space integrals are significantly smaller when the $K^{-}$data are included in the average.

KLOE, $\mathrm{KTeV}$, and NA48 also quote the values shown in Table 6 for $M_{V}$ from pole fits to $K_{L e 3}$ data. The average value of $M_{V}$ from all three experiments is $M_{V}=875 \pm 5 \mathrm{MeV}$ with $\chi^{2} / \mathrm{ndf}=1.8 / 2$. The three values are quite compatible with each other and reasonably close to the known value of the $K^{ \pm *}(892)$ mass $(891.66 \pm 0.26 \mathrm{MeV})$. The values for $\lambda_{+}^{\prime}$ and $\lambda_{+}^{\prime \prime}$ from expansion of the pole parametrization are qualitatively in agreement with the average of the quadratic fit results. More importantly, for the evaluation of the phase-space integrals, using the average of quadratic or pole fit results gives values of $I\left(K_{e 3}^{0}\right)$ that differ by just $0.03 \%$.

An attempt to estimate the theoretical uncertainties associated to form factor parameterization has been pursued by $\mathrm{KTeV}$, analyzing $K_{e 3}$ decays with the $z$-expansion 2.2.2 for the $f_{+}(t)$ form factor [47. The results are $a_{1} / a_{0}=1.023 \pm 0.040$ and $a_{2} / a_{0}=0.75 \pm 2.16$. The second order term is consistent with zero and the higher orders are bounded by the theory: $\sum_{k=0}^{\infty} a_{k}^{2} / a_{o}^{2} \leq 170$. Using these results the phase space integral is calculated to be 


\begin{tabular}{lc|c}
\hline \hline Experiment & $M_{V}(\mathrm{MeV})$ & $\left\langle M_{V}\right\rangle=875 \pm 5 \mathrm{MeV}$ \\
KLOE & $870 \pm 6 \pm 7$ & $\chi^{2} / \mathrm{ndf}=1.8 / 2$ \\
$\mathrm{KTeV}$ & $881.03 \pm 7.11$ & $\lambda_{+}^{\prime} \times 10^{3}=25.42(31)$ \\
$\mathrm{NA} 48$ & $859 \pm 18$ & $\lambda_{+}^{\prime \prime}=2 \times \lambda_{+}^{\prime 2}$ \\
& & $I\left(K_{e 3}^{0}\right)=0.15470(19)$ \\
\hline \hline
\end{tabular}

Table 6: Pole fit results for $K_{e 3}^{0}$ slopes.

$I\left(K_{e 3}^{0}\right)=0.15392 \pm 0.00048_{\text {exp }} \pm 0.00006_{\text {th }}$. The first error corresponds to the KTeV experimental uncertainty and the second error is due to possible effects from higher order terms in the $z$-expansion. Compared to the global average using the quadratic parameterization (Table 5), the $\mathrm{KTeV}$ measurement using the $z$-expansion deviates by about $1.5 \sigma_{\text {exp }}$. This result is less precise statistically, but it is more conservative as far as the estimate of the theoretical uncertainty is concerned.

\subsubsection{Scalar and Vector form factor slopes from $K_{\ell 3}$}

For $K_{\mu 3}$ decays, recent measurements of the slope parameters $\left(\lambda_{+}^{\prime}, \lambda_{+}^{\prime \prime}, \lambda_{0}\right)$ are available from KTeV [48], KLOE [52], ISTRA+ [53], and NA48 [54]. These data are summarized in Appendix D.

We have studied the statistical sensitivity of the form-factor slope measurements using Monte Carlo techniques, see Appendix E. The conclusions of this study are a) that neglecting a quadratic term in the parameterization of the scalar form factor when fitting results leads to a shift of the value of the linear term by about 3.5 times the value of the quadratic term; and b) that because of correlations, it is impossible to measure the quadratic slope parameter from quadratic fits to the data at any plausible level of statistics. The use of the linear representation of the scalar form factor is thus inherently unsatisfactory.

Figure 3 shows the 1- $\sigma$ contours from all the experimental results $\left(K_{e 3}\right.$ and $\left.K_{\mu 3}\right)$. It is immediately clear from the figure that the new NA48 results are difficult to accommodate 1. Performing the combination with and without the NA48 results for the $K_{\mu 3}$ form-factor slopes included we obtain fit probability values of $1 \times 10^{-6}$ and $22.3 \%$ respectively(see Appendix $\mathrm{D}$ for a detailed comparison). The results of the combination are listed in Table 7 .

The value of $\chi^{2} /$ ndf for all measurements is terrible; we quote the results with scaled errors. This leads to errors on the phase-space integrals that are $\sim 60 \%$ larger after inclusion of the new $K_{\mu 3}$ NA48 data.

We have checked to see if the NA48 $K_{\mu 3}$ data might show good consistency with the results of some other experiment in a less inclusive average. Fitting to only the $K_{\mu 3}$ results from $\mathrm{KTeV}, \mathrm{NA} 48$, and ISTRA + gives $\chi^{2} / \mathrm{ndf}=28 / 6(0.01 \%)$. Fitting to only the $K_{L \mu 3}$ results from $\mathrm{KTeV}, \mathrm{NA} 48$ gives $\chi^{2} / \mathrm{ndf}=12 / 3(0.89 \%)$. The consistency of the NA48 data with these other measurements appears to be poor in any case.

The evaluations of the phase-space integrals for all four modes are listed in each case. Correlations are fully accounted for, both in the fits and in the evaluation of the integrals.

\footnotetext{
${ }^{1}$ It lies out of correlation directions in the $\left[\lambda_{+}^{\prime}, \lambda_{+}^{\prime \prime}, \lambda_{0}\right]$ space
} 

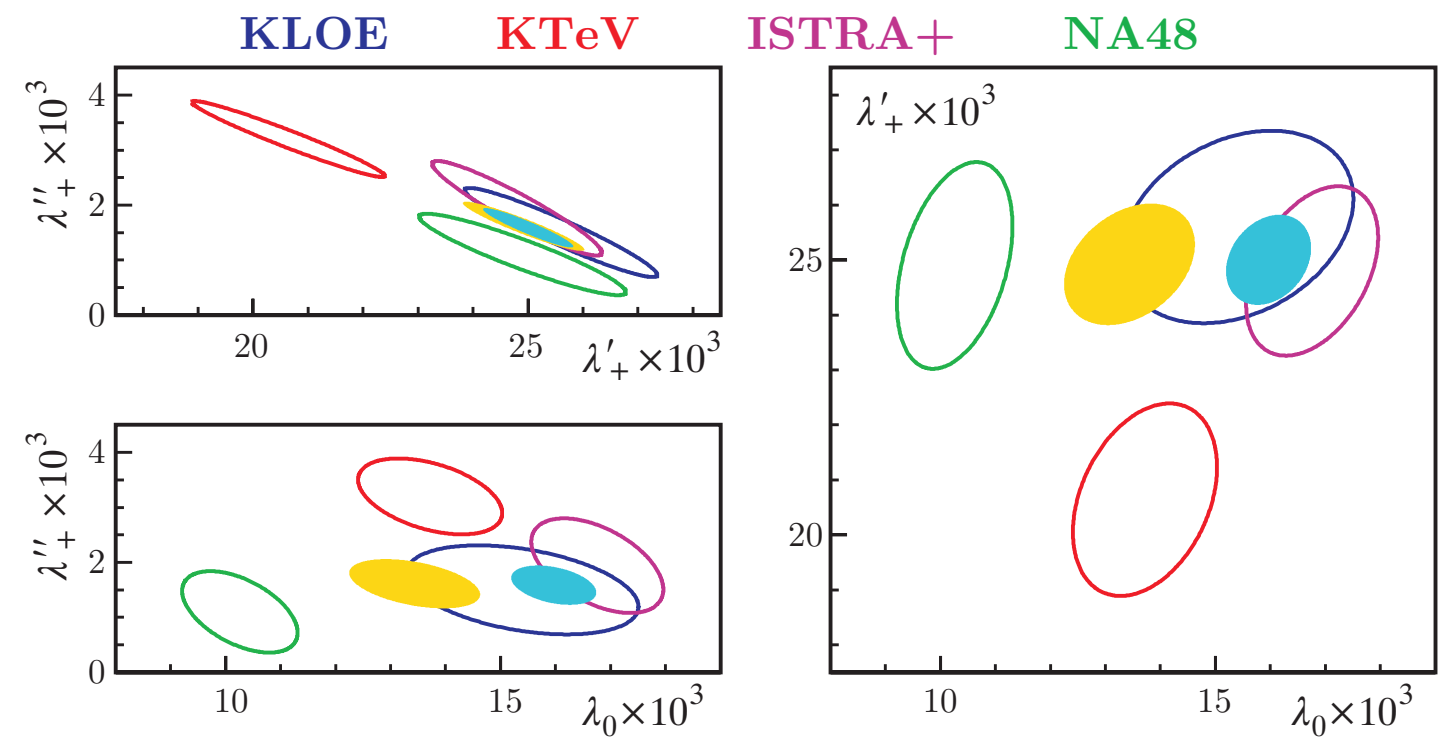

Figure 3: $1-\sigma$ contours for $\lambda_{+}^{\prime}, \lambda_{+}^{\prime \prime}, \lambda_{0}$ determinations from ISTRA+(pink ellipse), KLOE(blue ellipse), KTeV(red ellipse), NA48(green ellipse), and world average with(filled yellow ellipse) and without(filled cyan ellipse) the NA48 $K_{\mu 3}$ result.

\begin{tabular}{lcc}
\hline \hline & $K_{L}$ and $K^{-}$ & $K_{L}$ only \\
\hline Measurements & 16 & 11 \\
$\chi^{2} /$ ndf & $54 / 13\left(7 \times 10^{-7}\right)$ & $33 / 8\left(8 \times 10^{-5}\right)$ \\
$\lambda_{+}^{\prime} \times 10^{3}$ & $24.9 \pm 1.1(S=1.4)$ & $24.0 \pm 1.5(S=1.5)$ \\
$\lambda_{+}^{\prime \prime} \times 10^{3}$ & $1.6 \pm 0.5(S=1.3)$ & $2.0 \pm 0.6(S=1.6)$ \\
$\lambda_{0} \times 10^{3}$ & $13.4 \pm 1.2(S=1.9)$ & $11.7 \pm 1.2(S=1.7)$ \\
$\rho\left(\lambda_{+}^{\prime}, \lambda_{+}^{\prime \prime}\right)$ & -0.94 & -0.97 \\
$\rho\left(\lambda_{+}^{\prime}, \lambda_{0}\right)$ & +0.33 & +0.72 \\
$\rho\left(\lambda_{+}^{\prime \prime}, \lambda_{0}\right)$ & -0.44 & -0.70 \\
$I\left(K_{e 3}^{0}\right)$ & $0.15457(29)$ & $0.1544(4)$ \\
$I\left(K_{e 3}^{ \pm}\right)$ & $0.15892(30)$ & $0.1587(4)$ \\
$I\left(K_{\mu 3}^{0}\right)$ & $0.10212(31)$ & $0.1016(4)$ \\
$I\left(K_{\mu 3}^{ \pm}\right)$ & $0.10507(32)$ & $0.1046(4)$ \\
$\rho\left(I_{e 3}, I_{\mu 3}\right)$ & +0.63 & +0.89 \\
\hline \hline
\end{tabular}

Table 7: Averages of quadratic fit results for $K_{e 3}$ and $K_{\mu 3}$ slopes.

The correlation matrices for the integrals are of the form

$$
\begin{array}{cccc}
+1 & +1 & \rho & \rho \\
+1 & +1 & \rho & \rho \\
\rho & \rho & +1 & +1 \\
\rho & \rho & +1 & +1
\end{array}
$$

where the order of the rows and columns is $K_{e 3}^{0}, K_{e 3}^{ \pm}, K_{\mu 3}^{0}, K_{\mu 3}^{ \pm}$, and $\rho=\rho\left(I_{e 3}, I_{\mu 3}\right)$ as 


\begin{tabular}{l|c|c|c|c|c|c} 
mode & $\left|V_{u s}\right| \times f_{+}(0)$ & $\%$ err & BR & $\tau$ & $\Delta$ & Int \\
\hline$K_{L} \rightarrow \pi e \nu$ & $0.2163(6)$ & 0.28 & 0.09 & 0.19 & 0.15 & 0.09 \\
$K_{L} \rightarrow \pi \mu \nu$ & $0.2168(7)$ & 0.31 & 0.10 & 0.18 & 0.15 & 0.15 \\
$K_{S} \rightarrow \pi e \nu$ & $0.2154(13)$ & 0.67 & 0.65 & 0.03 & 0.15 & 0.09 \\
$K^{ \pm} \rightarrow \pi e \nu$ & $0.2173(8)$ & 0.39 & 0.26 & 0.09 & 0.26 & 0.09 \\
$K^{ \pm} \rightarrow \pi \mu \nu$ & $0.2176(11)$ & 0.51 & 0.40 & 0.09 & 0.26 & 0.15 \\
average & $0.2166(5)$ & & & & &
\end{tabular}

Table 8: Summary of $\left|V_{u s}\right| \times f_{+}(0)$ determination from all channels.

listed in the table.

Adding the $K_{\mu 3}$ data to the fit does not cause drastic changes to the values of the phase-space integrals for the $K_{e 3}$ modes: the values for $I\left(K_{e 3}^{0}\right)$ and $I\left(K_{e 3}^{ \pm}\right)$in Table 7 are qualitatively in agreement with those in Table 5 . As in the case of the fits to the $K_{e 3}$ data only, the significance of the quadratic term in the vector form factor is strong ( $3.6 \sigma$ from the fit to all data).

\section{Physics Results}

\subsection{Determination of $\left|V_{u s}\right| \times f_{+}(0)$ and $\left|V_{u s}\right| /\left|V_{u d}\right| \times f_{K} / f_{\pi}$}

This section describes the results that are independent on the theoretical parameters $f_{+}(0)$ and $f_{K} / f_{\pi}$.

\subsubsection{Determination of $\left|V_{u s}\right| \times f_{+}(0)$}

The value of $\left|V_{u s}\right| \times f_{+}(0)$ has been determined from (2.1) using the world average values reported in section 3 for lifetimes, branching ratios and phase space integrals, and the radiative and $S U(2)$ breaking corrections discussed in section 2 .

The results are given in Table 8, and are shown in Fig. 4 for $K_{L} \rightarrow \pi e \nu, K_{L} \rightarrow \pi \mu \nu$, $K_{S} \rightarrow \pi e \nu, K^{ \pm} \rightarrow \pi e \nu, K^{ \pm} \rightarrow \pi \mu \nu$, and for the combination. The average,

$$
\left|V_{u s}\right| \times f_{+}(0)=0.21664(48)
$$

has an uncertainty of about of $0.2 \%$. The results from the five modes are in good agreement, the fit probability is $58 \%$. In particular, comparing the values of $\left|V_{u s}\right| \times f_{+}(0)$ obtained from $K_{\ell 3}^{0}$ and $K_{\ell 3}^{ \pm}$we obtain a value of the $\mathrm{SU}(2)$ breaking correction

$$
\delta_{S U(2)_{\text {exp. }}}^{K}=2.9(4) \%
$$

in agreement with the CHPT calculation reported in Table 1: $\delta_{S U(2)}^{K}=2.36(22) \% .^{2}$

\footnotetext{
2 The value of $\delta_{S U(2)}^{K}$ has a direct correspondence to the ratio of light quark masses. Recent analyzes [55] on the so-called violations of Dashen's theorem in the Kaon electromagnetic mass splitting point to $\delta_{S U(2)}^{K}$ values of about $3 \%$ [56.
} 


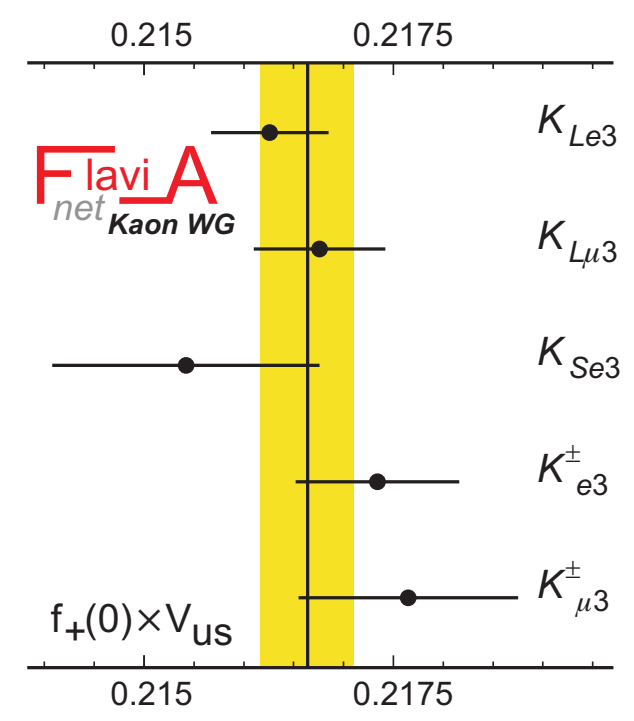

Figure 4: Display of $\left|V_{u s}\right| \times f_{+}(0)$ for all channels.

\subsubsection{Determination of $\left|V_{u s}\right| /\left|V_{u d}\right| \times f_{K} / f_{\pi}$}

An independent determination of $\left|V_{u s}\right|$ is obtained from $K_{\ell 2}$ decays. The most important mode is $K^{+} \rightarrow \mu^{+} \nu$, which has been recently updated by KLOE reaching a relative uncertainty of about $0.3 \%$. As shown in Eq. (2.2), hadronic uncertainties are minimized considering the ratio $\Gamma\left(K^{+} \rightarrow \mu^{+} \nu\right) / \Gamma\left(\pi^{+} \rightarrow \mu^{+} \nu\right)$.

Using the world average values of $\operatorname{BR}\left(K^{ \pm} \rightarrow \mu^{ \pm} \nu\right)$ and of $\tau^{ \pm}$given in Section 3 and the value of $\Gamma\left(\pi^{ \pm} \rightarrow \mu^{ \pm} \nu\right)=38.408(7) \mu s^{-1}$ from 433 we obtain:

$$
\left|V_{u s}\right| /\left|V_{u d}\right| \times f_{K} / f_{\pi}=0.2760 \pm 0.0006
$$

\subsection{The parameters $f_{+}(0)$ and $f_{K} / f_{\pi}$}

The main obstacle in transforming these highly precise determinations of $\left|V_{u s}\right| \times f_{+}(0)$ and $\left|V_{u s}\right| /\left|V_{u d}\right| \times f_{K} / f_{\pi}$ into a determination of $\left|V_{u s}\right|$ at the per-mil level are the theoretical uncertainties on the hadronic parameters $f_{+}(0)$ and $f_{K} / f_{\pi}$.

\subsubsection{Theoretical estimates of $f_{+}(0)$}

By construction, $f_{+}(0)$ is defined in the absence of isospin-breaking effects of both electromagnetic and quark-mass origin. More explicitly, as discussed in Section 2.1, $f_{+}(0)$ is defined by the $K^{0} \rightarrow \pi^{+}$matrix element of the vector current in the limit $m_{u}=m_{d}$ and $\alpha_{\mathrm{em}} \rightarrow 0$, keeping kaon and pion masses to their physical values.

This hadronic quantity cannot be computed in perturbative QCD, but it is highly constrained by $S U(3)$ and chiral symmetry. In the chiral limit and, more generally, in the $S U(3)$ limit $\left(m_{u}=m_{d}=m_{s}\right)$ the conservation of the vector current (CVC) implies $f_{+}(0)=1$. Expanding around the chiral limit in powers of light quark masses we can write

$$
f_{+}(0)=1+f_{2}+f_{4}+\ldots
$$




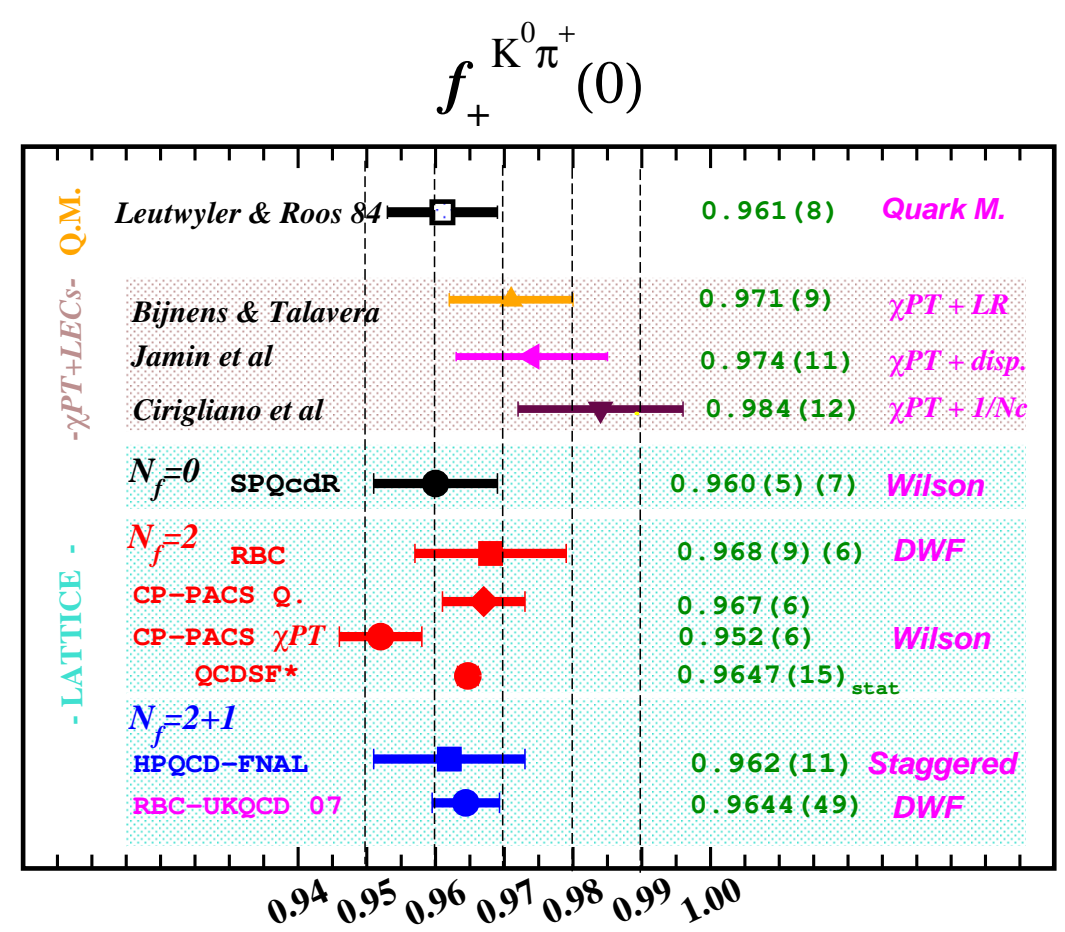

Figure 5: Present determinations of $f_{+}(0) \equiv f_{+}^{K^{0} \pi^{-}}(0)$ from lattice QCD and analytical or semianalytical approaches [57, 58, 59, 60].

where $f_{2}$ and $f_{4}$ are the NLO and NNLO corrections in ChPT. The Ademollo-Gatto theorem implies that $\left[f_{+}(0)-1\right]$ is at least of second order in the breaking of $S U(3)$ or in the expansion in powers of $m_{s}-\hat{m}$, where $\hat{m}=\left(m_{u}+m_{d}\right) / 2$. This in turn implies that $f_{2}$ is free from the uncertainties of the $\mathcal{O}\left(p^{4}\right)$ counterterms in ChPT, and it can be computed with high accuracy: $f_{2}=-0.023$ [57].

The difficulties in estimating $f_{+}(0)$ begin with $f_{4}$ or at $\mathcal{O}\left(p^{6}\right)$ in the chiral expansion. At this order we can write

$$
f_{4}=\Delta(\mu)+\left.f_{4}\right|^{\text {loc }}(\mu)
$$

where $\Delta(\mu)$ is the loop contribution, which has been computed in Ref. [18], and $\left.f_{4}\right|^{\text {loc }}(\mu)$ is the $\mathcal{O}\left(p^{6}\right)$ local contribution, whose knowledge cannot be simply deduced from other processes. Several analytical approaches to determine $f_{4}$ have been attempted over the years [60], essentially confirming the original estimate by Leutwyler and Roos [57] (see Fig. 5). The benefit of these new results, obtained using more sophisticated techniques, lies in the fact that a better control over the systematic uncertainties of the calculation has been obtained. However, the size of the error is still around or above $1 \%$, which is not comparable to the $0.2 \%$ accuracy which has been reached for $\left|V_{u s}\right| \times f_{+}(0)$.

Recent progress in lattice QCD gives us more optimism in the reduction of the error on $f_{+}(0)$ below the $1 \%$ level [62, 61, 63]. Most of the currently available lattice QCD results have been obtained with relatively heavy pions and the chiral extrapolation represents the dominant source of uncertainty. As shown in Figure 5, there is a general trend of lattice QCD results to be slightly lower than analytical approaches. An important step in the 


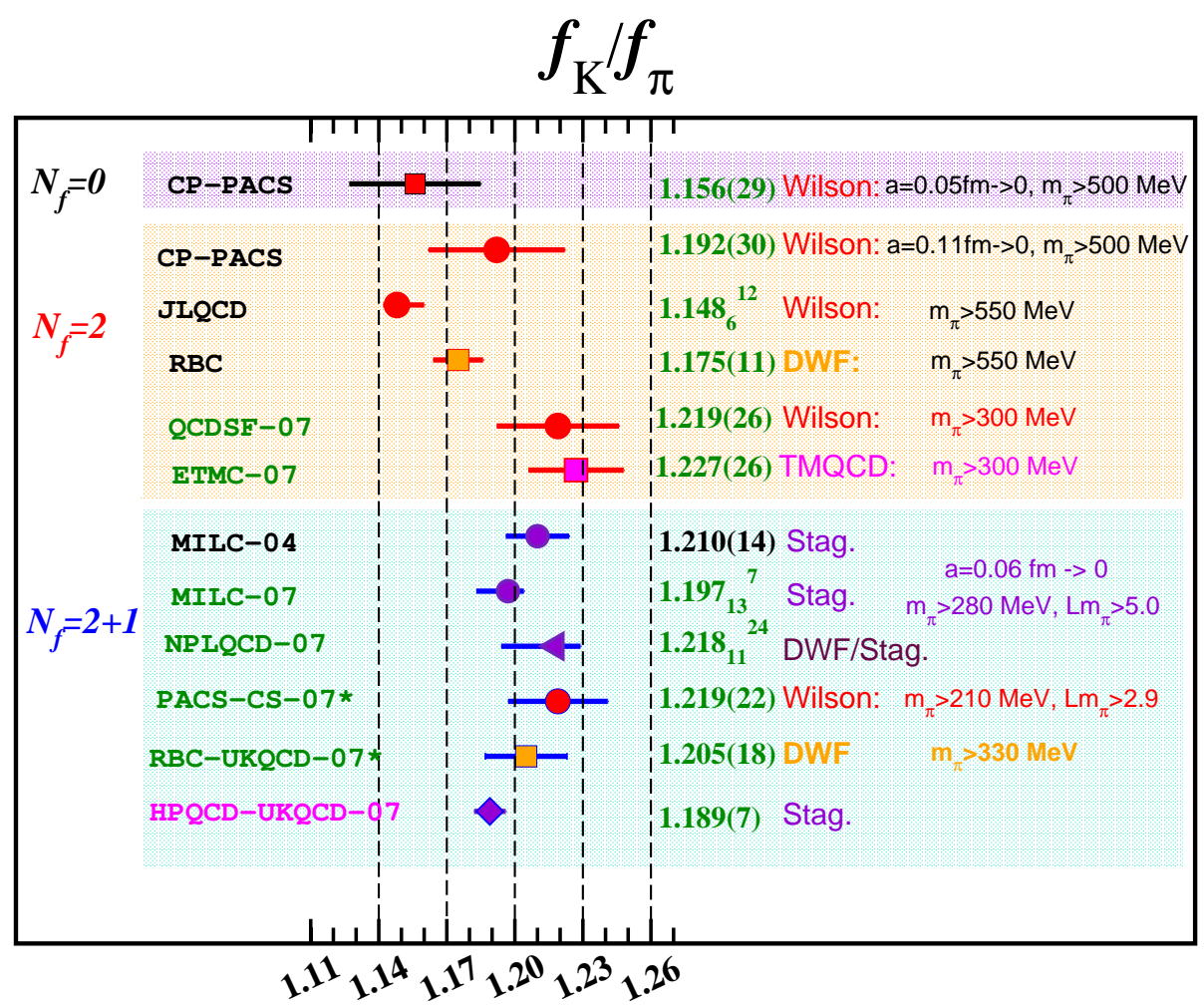

Figure 6: Summary of $f_{K} / f_{\pi}$ estimates 64, 65, 66, 67]. All values are from Lattice QCD. In recent studies, sea quarks are getting light and data are matched to ChPT fits to determine the Low-energy-Constants (LEC).

reduction of the error associated to the chiral extrapolation has been recently made by the UKQCD-RBC collaboration 58]. Their preliminary result $f_{+}(0)=0.964(5)$ is obtained from the unquenched study with $N_{F}=2+1$ flavors, with an action that has good chiral properties on the lattice even at finite lattice spacing (domain-wall quarks). They also reached pions masses $(\gtrsim 330 \mathrm{MeV})$ much lighter than that used in previous studies of $f_{+}(0)$. The overall error is estimated to be $0.5 \%$, which is very encouraging. Moreover, they observe for $f_{+}(0)$ a mass dependence similar to the one of $f_{2}$. That is something new with respect to previous lattice studies (this is likely due to the fact that they work with lighter pions). To assess the chiral uncertainty of $f_{4}$, polynomial fits (linear and quadratic) well reproduce the data. However, it would be interesting [61] to have the expression of $\Delta(\mu)$ in Eq. (4.4) in terms of the quark masses so to directly estimate $\left.f_{4}\right|^{\text {loc }}(\mu)$. Moreover, it should also be stressed that the present study is performed at a single value of the lattice spacing $(a=0.12 \mathrm{fm})$ and in a relatively small extension of the fifth dimension of the lattice. $^{3}$

In the following phenomenological analysis we will use this result as the present best estimate of $f_{+}(0)$, although some reservation remains.

\footnotetext{
${ }^{3}$ Even though $m_{\pi} L \gtrsim 4.5$, simulations with a larger fifth dimension, $L_{s}$ would help too because the mass of their lightest quark $(=0.005$ in lattice units) is very close to the residual mass parameter $(=0.003$, also in lattice units). This may entail some uncontrolled systematics, in particular for $f_{K} / f_{\pi}$
} 


\subsubsection{Theoretical estimates of $f_{K} / f_{\pi}$}

In contrast to the semileptonic vector form factor, the pseudoscalar decay constants are not protected by the Ademollo-Gatto theorem and receive corrections linear in the quark masses. Expanding $f_{K} / f_{\pi}$ in power of quark masses, in analogy to $f_{+}(0)$,

$$
f_{K} / f_{\pi}=1+r_{2}+\ldots
$$

one finds that the $\mathcal{O}\left(p^{4}\right)$ contribution $r_{2}$ is already affected by local contributions and cannot be unambiguously predicted in ChPT. As a result, in the determination of $f_{K} / f_{\pi}$ lattice QCD [64]-67] has essentially no competition from purely analytical approaches. The status of the lattice results for $f_{K} / f_{\pi}$ is summarized in Fig. 6. As can be seen, the present overall accuracy is about $1 \%$. The novelty are the new lattice results with $N_{F}=2+1$ dynamical quarks and pions as light as $280 \mathrm{MeV}$ 64, 65], obtained by using the so-called staggered quarks. ${ }^{4}$ The analyzes of [64, 65], cover a broad range of lattice spacings (i.e. $a=0.06$ and $0.15 \mathrm{fm}$ ) and is performed on sufficiently large physical volumes $\left(m_{\pi} L \gtrsim 5.0\right)$. It should be stressed, however, that the sensitivity of $f_{K} / f_{\pi}$ to lighter pions is larger than in the computation of $f_{+}(0)$ and that chiral extrapolations are far more demanding in this case. ${ }^{5}$ Notice also that at Lattice 2007 preliminary studies with $N_{F}=$ $2+1$ clover quarks and pion masses $\gtrsim 200 \mathrm{MeV}$ have been presented from either PACS-CS Collaboration [70] and ref. [71]. With respect to the results obtained with staggered quarks, the PACS-CS value of $f_{K} / f_{\pi}$ in fig. 6 is restricted to a single lattice spacing $(a=0.09 \mathrm{fm})$ and relatively small physical volume $\left(m_{\pi} L \gtrsim 2.9\right)$. For ref. [71], the final analysis is to be completed. In the following analysis we will use as reference value the MILC-HPQCD result $f_{K} / f_{\pi}=1.189(7)$ [65], although some reservation about staggered fermions remains.

\subsubsection{A test of lattice calculation: the Callan-Treiman relation}

As described in Sect. 2.2 the Callan-Treiman relation fixes the value of scalar form factor at $t=m_{K}^{2}-m_{\pi}^{2}$ (the so-called Callan-Treiman point) to the ratio $\left(f_{K} / f_{\pi}\right) / f_{+}(0)$. The dispersive parametrization for the scalar form factor proposed in [12 and discussed in

\footnotetext{
${ }^{4}$ Staggered fermions come in four tastes on the lattice. In the continuum limit the extra degrees of freedom decouple from physical predictions. But, at finite lattice spacing, where the data are produced, the taste symmetry is violated and the extra degrees of freedom are removed by hand, namely by taking the fourth root of the staggered quark determinant. Theoretically, this procedure has been only confirmed in perturbation theory and is currently a subject of controversies within the lattice QCD community [68]. Since the staggered dynamical quarks are computationally cheap, they have been largely used. Thanks to recent progress in algorithm building [69], safer but still computationally competitive alternatives are becoming available.

${ }^{5}$ In some details, effects of chiral logs are not clearly disentangled and analytic terms (NNLO or NNNLO) are still needed in order to extrapolate from the simulated sea quark masses ( such as $m_{\pi} \gtrsim 280 \mathrm{MeV}$ ) to the physical point. For example, the two studies of ref. [64] and of ref. 65] with staggered quarks share the same configurations, but they differ in how to extrapolate to the physical masses. Then, the central values of $f_{K} / f_{\pi}$ between the two analyzes (namely, $f_{K} / f_{\pi}=1.197_{13}^{7}$ and $f_{K} / f_{\pi}=1.189(7)$ from ref. 64 and ref. 655 respectively) differ for $1 \sigma$. However, taking into account the complete uncertainty of $f_{K} / f_{\pi}$ in [64], we have $f_{K} / f_{\pi}=1.194(10)$ of [64] for a symmetric error and the values of ref. 64] and ref. 65 look now in good agreement. The highly improved staggered fermions (HISQ) used in [65] for the valence quarks are designed to reduce the taste violation effects, which also should reduce the overall systematic uncertainty.
} 


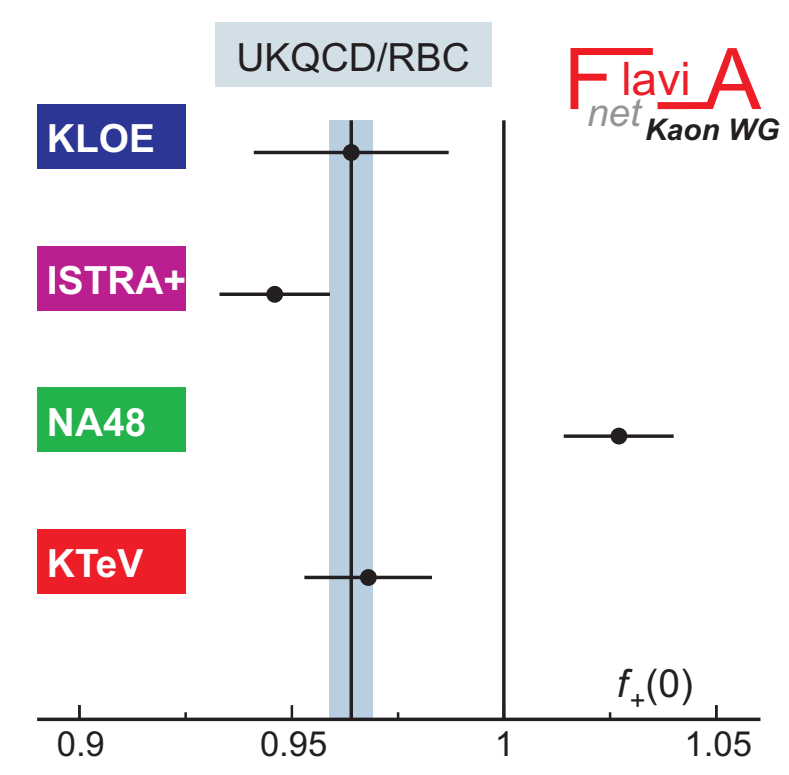

Figure 7: Values for $f_{+}(0)$ determined from the scalar form factor slope using the Callan-Treiman relation and $f_{K} / f_{\pi}=1.189(7)$. The UKQCD/RBC result $f_{+}(0)=0.964(5)$ is also shown.

Sect. 2.2 allows to transform the available measurements of the scalar form factor into a precise information on $\left(f_{K} / f_{\pi}\right) / f_{+}(0)$, completely independent of the lattice estimates.

Very recently KLOE [52] and NA48 [54] have presented results on the scalar FF slope using the dispersive parametrization. In these analyzes a dispersive parametrization is used for both the scalar and the vector form factors. A similar analysis has started for the $\mathrm{KTeV}$ data. We report these preliminary results for the first time. The ISTRA+ measurement of the scalar form factor slope performed using the first order Taylor expansion parametrization can be translated in the dispersive parametrization as described in Appendix $\mathbb{E}$. The results are given in Table 9 for all the four experiments in the case of the pole parametrization for the vector form factor. The original KLOE and NA48 results are also shown for comparison as well as the preliminary result of $\mathrm{KTeV}$ obtained from the $K_{\mu 3}$ data analysis. Moreover, a combined $K_{e 3}$ and $K_{\mu 3}$ data analysis is also in progress and the preliminary result is: $\log (C)=0.191 \pm 0.012$. The preliminary $\mathrm{KTeV}$ results are obtained using the original MC and data from Ref. [48]. .

\begin{tabular}{l|c|c} 
Experiment & $\log (C)$ direct & $\log (C)^{\dagger}$ \\
\hline KTeV & $0.195(14)^{*}$ & $0.203(15)$ \\
KLOE & $0.207(24)$ & $0.207(23)$ \\
NA48 & $0.144(14)$ & $0.144(13)$ \\
ISTRA+ & & $0.226(13)$ \\
\hline
\end{tabular}

$\dagger$ Estimated from $\lambda_{0}$ published. ${ }^{*}$ Preliminary results.

Table 9: Experimental results for $\log (\mathrm{C})$.

Figure 7 shows the values for $f_{+}(0)$ determined from the scalar form factor slope mea- 
surements obtained using the Callan-Treiman relation and $f_{K} / f_{\pi}=1.189(7)$. The value of $f_{+}(0)=0.964(5)$ from UKQCD/RBC is also shown. As already noticed in Section 3, the NA48 result is difficult to accommodate. Here one can see that this results is also not consistent with the theoretical estimates of $f_{+}(0)$. In particular, it violates the Fubini-Furlan bound $f_{+}(0)<1$ [72]. For this reason, the NA48 result will be excluded when using the Callan-Treiman constraint.

The average of the experimental results on the FFs with the pole parametrization for the vector case and the dispersive parametrization for the scalar FF give:

$$
\begin{aligned}
& \lambda_{+}^{c}=0.0256 \pm 0.0002, \\
& \lambda_{0}^{c}=0.0149 \pm 0.0007,
\end{aligned}
$$

with correlation coefficient -0.32 . The above results are then combined with the lattice determinations of $f_{K} / f_{\pi}=1.189(7)$ and $f_{+}(0)=0.964(5)$ using the constraint given by the Callan-Treiman relation. The results of the combination are given in Table 10, where $\log C=\lambda_{0}^{c} t_{\mathrm{CT}} / m_{\pi}^{2}+0.0398 \pm 0.0041$.

\begin{tabular}{c|c|c|c}
$\lambda_{+}^{c}$ & $\lambda_{0}^{c}$ & $f_{+}(0)$ & $f_{K} / f_{\pi}$ \\
\hline $0.02563(19)$ & $0.0146(5)$ & $0.96(4)$ & $1.192(6)$ \\
\hline \multicolumn{4}{|c}{ correlation matrix } \\
\hline 1. & -0.23 & 0.12 & -0.14 \\
& 1. & -0.51 & 0.61 \\
& & 1. & 0.30 \\
& & & 1.
\end{tabular}

Table 10: Results from the form factor fit.

The fit probability is $39 \%$, confirming the agreement between experimental measurements and lattice determination. The accuracy of $f_{K} / f_{\pi}$ is also slightly improved, and this effect can be better seen in the ratio $f_{+}(0) /\left(f_{K} / f_{\pi}\right)$, directly related to the Callan-Treiman constraint.

As previously discussed, new physics contributions to the scalar form factor (reabsorbed into the value of $\log C$ ) are generated only by scalar operators. Hence in the case of right-handed currents $\log C$ coincides with the SM value. This imply we can use the Callan-Treiman improved $f_{+}(0) /\left(f_{K} / f_{\pi}\right)$ in constraining right-handed currents. On the other hand, this is not possible in the MSSM scenario, where scalar operators are present. Here the measured value of $\log C$, following from (2.53), is

$$
\log C^{\mathrm{MSSM}} \equiv \log \left|f_{0}^{H}\left(t_{C T}\right) / f_{0}(0)\right|=\log C^{\mathrm{SM}}-r_{H}^{K}
$$

with the $r_{H}^{K}$ given in (2.39). By construction, the quantity $\log C^{\mathrm{SM}}$ depends only on QCD dynamics and must satisfies the Callan-Treiman relation (2.8). The theoretical calculation of $f_{0}\left(t_{C T}\right)$ can thus be used to constrain scalar densities. At present, the theoretical knowledge of $\log C^{\mathrm{SM}}$ is obtained from Eq. (2.8) and is limited by our knowledge of $\Delta_{C T}$, 


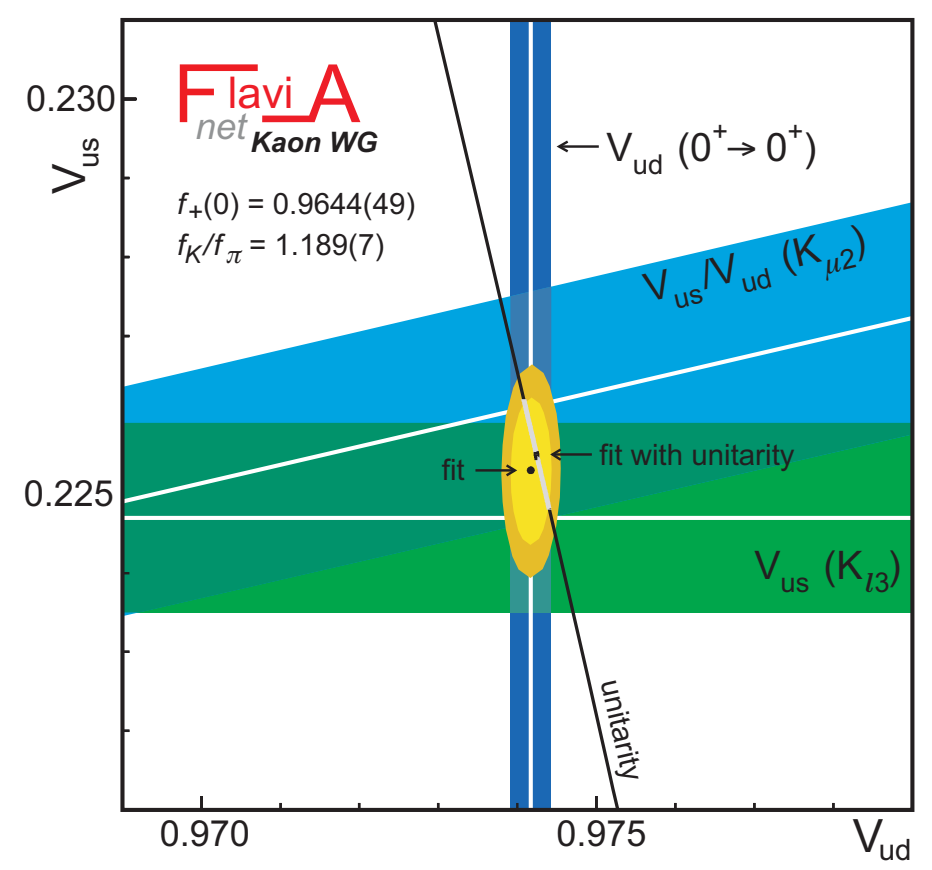

Figure 8: Results of fits to $\left|V_{u d}\right|,\left|V_{u s}\right|$, and $\left|V_{u s}\right| /\left|V_{u d}\right|$.

reported in (2.9), and by the lattice QCD results on $\left(f_{K} / f_{\pi}\right) / f_{+}(0)$. Using this information we obtain the constraint

$$
r_{H}^{K}=-0.007 \pm 0.012
$$

To improve this result it would be particularly useful a direct computation of $\left(f_{K} / f_{\pi}\right) / f_{+}(0)$ on the lattice (i.e. from the the same set of simulations). Given the advanced status of staggered results on $f_{K} / f_{\pi}$, it would be interesting to see the effect of a corresponding analysis $f_{+}(0)$ (which at present is still very preliminary [59]).

\subsection{Test of Cabibbo Universality or CKM unitarity}

To determine $\left|V_{u s}\right|$ and $\left|V_{u d}\right|$ we use the value $\left|V_{u s}\right| \times f_{+}(0)=0.2166(5)$ reported in Table 8, the result $\left|V_{u s}\right| /\left|V_{u d}\right| f_{K} / f_{\pi}=0.2760(6)$ discussed in Sect. 4.1.2, $f_{+}(0)=0.964(5)$, and $f_{K} / f_{\pi}=1.189(7)$. From the above we find:

$$
\begin{aligned}
\left|V_{u s}\right| & =0.2246 \pm 0.0012 & & {\left[K_{\ell 3} \text { only }\right], } \\
\left|V_{u s}\right| /\left|V_{u d}\right| & =0.2321 \pm 0.0015 & & {\left[K_{\ell 2} \text { only }\right] . }
\end{aligned}
$$

These determinations can be used in a fit together with the the recent evaluation of $\left|V_{u d}\right|$ from $0^{+} \rightarrow 0^{+}$nuclear beta decays: $\left|V_{u d}\right|=0.97418 \pm 0.00026$ [73]. The global fit gives

$$
\left|V_{u d}\right|=0.97417(26) \quad\left|V_{u s}\right|=0.2253(9) \quad\left[K_{\ell 3, \ell 2}+0^{+} \rightarrow 0^{+}\right],
$$

with $\chi^{2} /$ ndf $=0.65 / 1(42 \%)$. This result does not make use of CKM unitarity. If the unitarity constraint is included, the fit gives

$$
\left|V_{u s}\right|=\sin \theta_{C}=\lambda=0.2255(7) \quad \text { [with unitarity] }
$$



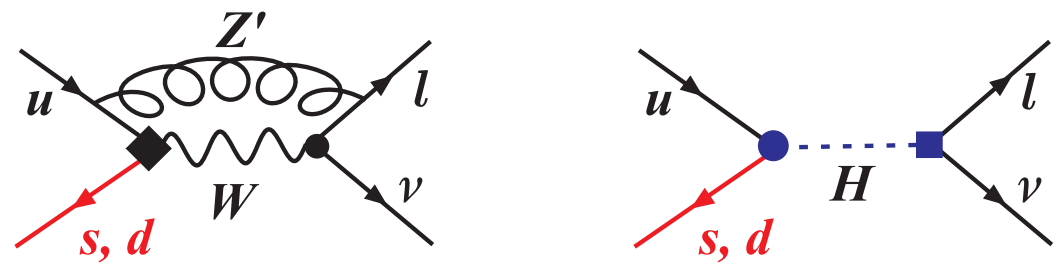

Figure 9: $\quad Z^{\prime}$ and Higgs exchange.

and $\chi^{2} /$ ndf $=0.80 / 2(67 \%)$. Both results are illustrated in Fig. 8 .

As described in the introduction, the test of CKM unitarity can be also interpreted as a test of universality of the lepton and quark gauge couplings. Using the results of the fit (without imposing unitarity) we obtain:

$$
G_{\mathrm{CKM}} \equiv G_{\mu}\left[\left|V_{u d}\right|^{2}+\left|V_{u s}\right|^{2}+\left|V_{u b}\right|^{2}\right]^{1 / 2}=(1.1662 \pm 0.0004) \times 10^{-5} \mathrm{GeV}^{-2},
$$

in perfect agreement with the value obtained from the measurement of the muon lifetime:

$$
G_{\mu}=(1.166371 \pm 0.000007) \times 10^{-5} \mathrm{GeV}^{-2} .
$$

The current accuracy of the lepton-quark universality sets important constraints on model building beyond the SM. For example, the presence of a $Z^{\prime}$ (see Fig. 9, left) would affect the relation between $G_{\mathrm{CKM}}$ and $G_{\mu}$ in the following way,

$$
G_{\mu}=G_{C K M}\left[1-0.007 Q_{e L}\left(Q_{\mu L}-Q_{d L}\right) \frac{2 \ln \left(m_{Z^{\prime}} / m_{W}\right)}{m_{Z^{\prime}}^{2} / m_{W}^{2}-1}\right],
$$

where $Q_{f L}$ are the generic charges of the $Z^{\prime}$ to left-handed leptons (in units of the SM $S U(2)_{L}$ charge). In case of a $Z^{\prime}$ from $S O(10)$ grand unification theories $\left(Q_{e L}=Q_{\mu L}=\right.$ $\left.-3 Q_{d L}=1\right)$ we obtain $m_{Z^{\prime}}>700 \mathrm{GeV}$ at $95 \% \mathrm{CL}$, to be compared with the $m_{Z^{\prime}}>720 \mathrm{GeV}$ bound set through the direct collider searches [43]. In a similar way, the unitarity constraint also provides useful bounds in various supersymmetry-breaking scenarios 74 .

\subsubsection{Bounds on helicity-suppressed amplitudes}

A particularly interesting test is the comparison of the $\left|V_{u s}\right|$ value extracted from the helicity-suppressed $K_{\ell 2}$ decays with respect to the value extracted from the helicity-allowed $K_{\ell 3}$ modes. To reduce theoretical uncertainties from $f_{K}$ and electromagnetic corrections in $K_{\ell 2}$, we exploit the ratio $\operatorname{Br}\left(K_{\ell 2}\right) / B r\left(\pi_{\ell 2}\right)$ and we study the quantity

$$
R_{l 23}=\left|\frac{V_{u s}\left(K_{\ell 2}\right)}{V_{u s}\left(K_{\ell 3}\right)} \times \frac{V_{u d}\left(0^{+} \rightarrow 0^{+}\right)}{V_{u d}\left(\pi_{\ell 2}\right)}\right| .
$$

Within the SM, $R_{l 23}=1$, while deviation from 1 can be induced by non-vanishing scalaror right-handed currents. Notice that in $R_{l 23}$ the hadronic uncertainties enter through $\left(f_{K} / f_{\pi}\right) / f_{+}(0)$.

Following the notation of Section 2.3, effects of scalar currents due to a charged Higgs (Fig. 9 right) give

$$
R_{l 23}=\left|1-\frac{m_{K^{+}}^{2}}{M_{H^{+}}^{2}}\left(1-\frac{m_{d}}{m_{s}}\right) \frac{\tan ^{2} \beta}{1+\epsilon_{0} \tan \beta}\right| \text {, }
$$




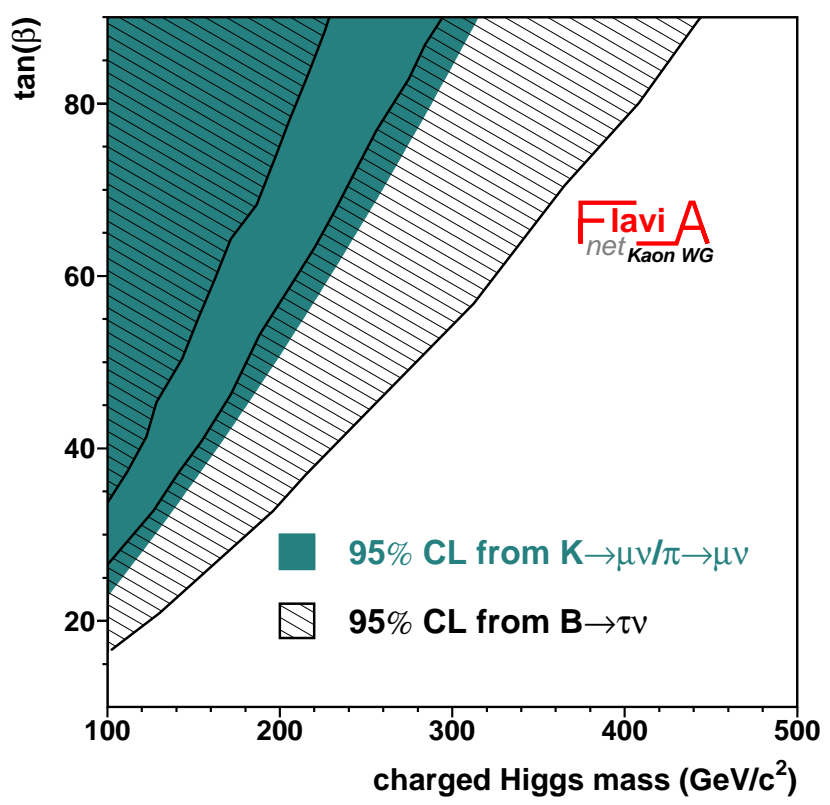

Figure 10: Excluded region in the charged Higgs mass-tan $\beta$ plane. The region excluded by $B \rightarrow \tau \nu$ is also indicated.

whereas for right-handed currents we have

$$
R_{l 23}=1-2\left(\epsilon_{s}-\epsilon_{n s}\right)
$$

In the case of scalar densities (MSSM), the unitarity relation between $\left|V_{u d}\right|$ extracted from $0^{+} \rightarrow 0^{+}$nuclear beta decays and $\left|V_{u s}\right|$ extracted from $K_{\ell 3}$ remains valid as soon as form factors are experimentally determined. This constrain together with the experimental information of $\log C^{M S S M}$ can be used in the global fit to improve the accuracy of the determination of $R_{l 23}$, which in this scenario turns to be

$$
\left.R_{l 23}\right|_{\mathrm{scalar}} ^{\exp }=1.004 \pm 0.007 .
$$

Here $\left(f_{K} / f_{\pi}\right) / f_{+}(0)$ has been fixed from lattice. This ratio is the key quantity to be improved in order to reduce present uncertainty on $R_{l 23}$.

The measurement of $R_{l 23}$ above can be used to set bounds on the charged Higgs mass and $\tan \beta$. Figure 10 shows the excluded region at 95\% CL in the $M_{H}$-tan $\beta$ plane (setting $\left.\epsilon_{0}=0.01\right)$. The measurement of $\operatorname{BR}(B \rightarrow \tau \nu)$ 75 can be also used to set a similar bound in the $M_{H}-\tan \beta$ plane. While $B \rightarrow \tau \nu$ can exclude quite an extensive region of this plane, there is an uncovered region in the exclusion corresponding to a destructive interference between the charged-Higgs and the SM amplitude. This region is fully covered by the $K \rightarrow \mu \nu$ result.

In the case of right-handed currents [12], $R_{l 23}$ can be obtained from a global fit to the values of eqs. (4.1) and (4.2). Here $\log C^{\exp }$ is free of new physics effects and can be 
also used to constrain $\left(f_{K} / f_{\pi}\right) / f_{+}(0)$ together with lattice results (namely the values in tab. 10). The result is

$$
\left.R_{l 23}\right|_{\text {RHcurr. }} ^{\exp }=1.004 \pm 0.006
$$

\subsection{Tests of Lepton Flavor Universality}

\subsubsection{Lepton universality in $K_{\ell 3}$ decays}

The test of Lepton Flavor Universality (LFU) between $K_{e 3}$ and $K_{\mu 3}$ modes constraints a possible anomalous lepton-flavor dependence in the leading weak vector current. It can therefore be compared to similar tests in $\tau$ decays, but is different from the LFU tests in the helicity-suppressed modes $\pi_{l 2}$ and $K_{l 2}$.

The results on the parameter $r_{\mu e}=R_{K_{\mu 3} / K_{e 3}}^{\mathrm{Exp}} / R_{K_{\mu 3} / K_{e 3}}^{\mathrm{SM}}$ is

$$
r_{\mu e}=1.004 \pm 0.004
$$

in excellent agreement with lepton universality. Furthermore, with a precision of $0.5 \%$ the test in $K_{l 3}$ decays has now reached the sensitivity of $\tau$ decays.

\subsubsection{Lepton universality tests in $K_{\ell 2}$ decays}

The ratio $R_{K}=\Gamma\left(K_{\mu 2}\right) / \Gamma\left(K_{e 2}\right)$ can be precisely calculated within the Standard Model. Neglecting radiative corrections, it is given by

$$
R_{K}^{(0)}=\frac{m_{e}^{2}}{m_{\mu}^{2}} \frac{\left(m_{K}^{2}-m_{e}^{2}\right)^{2}}{\left(m_{K}^{2}-m_{\mu}^{2}\right)^{2}}=2.569 \times 10^{-5},
$$

and reflects the strong helicity suppression of the electron channel. Radiative corrections have been computed with effective theories [6], yielding the final SM prediction

$$
\begin{aligned}
R_{K}^{\mathrm{SM}} & =R_{K}^{(0)}\left(1+\delta R_{K}^{\text {rad.corr. }}\right) \\
& =2.569 \times 10^{-5} \times(0.9622 \pm 0.0004)=(2.477 \pm 0.001) \times 10^{-5}
\end{aligned}
$$

Because of the helicity suppression within then SM, the $K_{e 2}$ amplitude is a prominent candidate for possible sizable contributions from physics beyond the SM. Moreover, when normalizing to the $K_{\mu 2}$ rate, we obtain an extremely precise prediction of the $K_{e 2}$ width within the SM. In order to be visible in the $K_{e 2} / K_{\mu 2}$ ratio, the new physics must violate lepton flavor universality.

Recently it has been pointed out that in a supersymmetric framework sizable violations of lepton universality can be expected in $K_{l 2}$ decays [26]. At the tree level, lepton flavor violating terms are forbidden in the MSSM. However, these appear at the one-loop level, where an effective $H^{+} l \nu_{\tau}$ Yukawa interaction is generated. Following the notation of Ref. [26] (see also Section 2.3), the non-SM contribution to $R_{K}$ can be written as

$$
R_{K}^{\mathrm{LFV}} \approx R_{K}^{\mathrm{SM}}\left[1+\left(\frac{m_{K}^{4}}{M_{H^{ \pm}}^{4}}\right)\left(\frac{m_{\tau}^{2}}{m_{e}^{2}}\right)\left|\Delta_{13}\right|^{2} \tan ^{6} \beta\right] .
$$

The lepton flavor violating coupling $\Delta_{13}$, being generated at the loop level, could reach values of $\mathcal{O}\left(10^{-3}\right)$. For moderately large $\tan \beta$ values, this contribution may therefore 


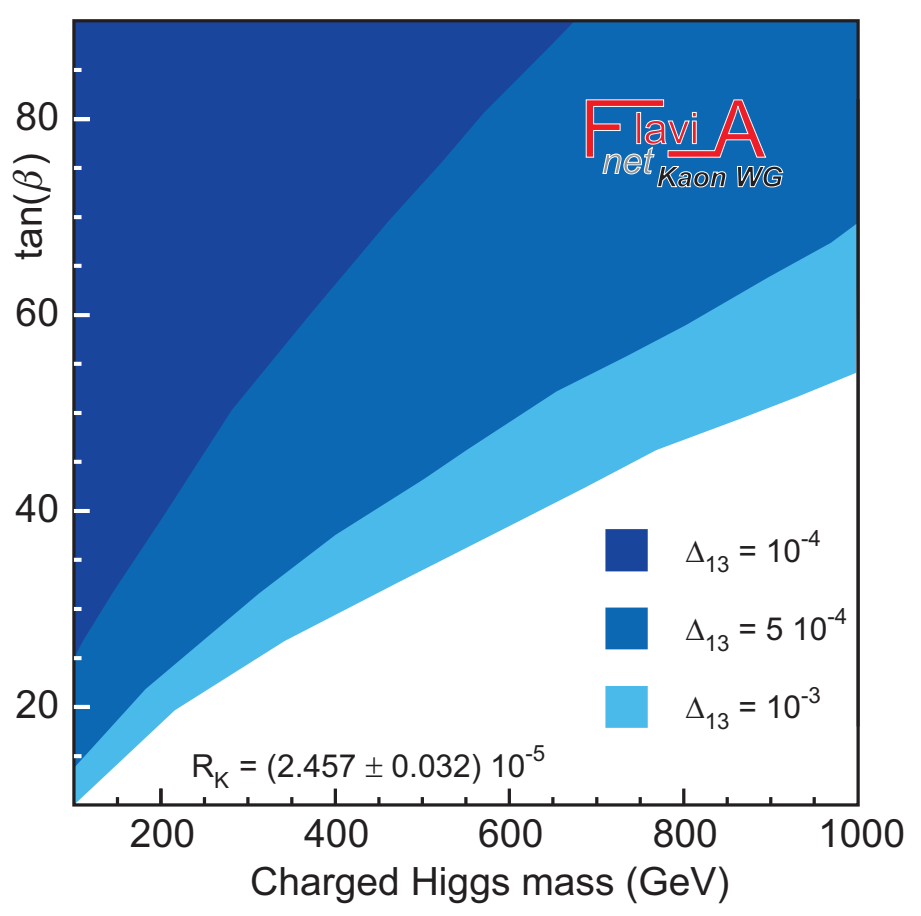

Figure 11: Exclusion limits at 95\% CL on $\tan \beta$ and the charged Higgs mass $M_{H^{ \pm}}$from $\left|V_{u s}\right|_{K \ell 2} /\left|V_{u s}\right|_{K \ell 3}$ for different values of $\Delta_{13}$.

enhance $R_{K}$ by up to a few percent. Since the additional term in Eq. 4.24 goes with the forth power of the meson mass, no similar effect is expected in $\pi_{l 2}$ decays.

The world average result for $R_{K}$ presented in Section 3 gives strong constraints for $\tan \beta$ and $M_{H^{ \pm}}$, as shown in Fig. 11. For values of $\Delta_{13} \approx 5 \times 10^{-4}$ and $\tan \beta>50$ the charged Higgs masses is pushed above $1000 \mathrm{GeV} / c^{2}$ at $95 \% \mathrm{CL}$.

\section{Acknowledgments}

We thank all the members of the FlaviaNet Kaon Working Group [www.Inf.infn.it/wg/vus], and in particular J. Gasser and J. Stern, for comments, discussions, and suggestions. This work is supported in part by the EU contract No. MTRN-CT-2006-035482 (FlaviaNet).

\section{References}

[1] N. Cabibbo, Phys. Rev. Lett. 10 (1963) 531; M. Kobayashi and T. Maskawa, Prog. Th. Phys 49 (1973) 652.

[2] D. B. Chitwood et al. [MuLan Collaboration], Phys. Rev. Lett. 99 (2007) 032001 [arXiv:0704.1981 [hep-ex]].

[3] E. Blucher et al., Status of the Cabibbo angle, arXiv:hep-ph/0512039.

[4] A. Sirlin, Nucl. Phys. B 196, 83 (1982).

[5] W. J. Marciano, Phys. Rev. Lett. 93, 231803 (2004) [arXiv:hep-ph/0402299]. 
[6] V. Cirigliano and I. Rosell, JHEP 0710, 005 (2007), arXiv:0707.4464 [hep-ph]; arXiv:0707.3439 [hep-ph].

[7] V. Cirigliano et al., Eur. Phys. J. C 35 (2004) 53; Eur. Phys. J. C 23 (2002) 121; T. C. Andre, hep-ph/0406006.

[8] V. Cirigliano, M. Giannotti, and H. Neufeld, work in preparation.

[9] R. J. Hill, Phys. Rev. D 74, 096006 (2006) [arXiv:hep-ph/0607108].

[10] B. Moussallam, arXiv:0710.0548 [hep-ph].

[11] V. Bernard, M. Oertel, E. Passemar and J. Stern in preparation; E. Passemar, Kaon International Conference (KAON 2007), PoS(KAON)012 (2007) [arXiv:0708.1235 [hep-ph]]

[12] V. Bernard, M. Oertel, E. Passemar and J. Stern, arXiv:0707.4194 [hep-ph]; Phys. Lett. B 638, 480 (2006) [arXiv:hep-ph/0603202].

[13] D. Becirevic, V. Lubicz, G. Martinelli and F. Mescia [SPQcdR Collaboration], Phys. Lett. B 501, 98 (2001) [arXiv:hep-ph/0010349].

[14] G. Colangelo, G. Isidori and J. Portoles, Phys. Lett. B 470, 134 (1999) [arXiv:hep-ph/9908415].

[15] J. Gasser and H. Leutwyler, Nucl. Phys. B 250 (1985) 517.

[16] H. Leutwyler, private communication.

[17] J. Bijnens and K. Ghorbani, arXiv:0711.0148 [hep-ph].

[18] J. Bijnens and P. Talavera, Nucl. Phys. B 669 (2003) 341 [arXiv:hep-ph/0303103].

[19] V. Bernard and E. Passemar, arXiv:0711.3450 [hep-ph].

[20] R. J. Hill, Phys. Rev. D 74, 096006 (2006) [arXiv:hep-ph/0607108].

[21] T. Becher and R. J. Hill, Phys. Lett. B 633, 61 (2006).

[22] R. Barate et al. [ALEPH Collaboration], Eur. Phys. J. C 11, 599 (1999).

[23] C. Bourrely, B. Machet and E. de Rafael, Nucl. Phys. B 189, 157 (1981). C. G. Boyd, B. Grinstein and R. F. Lebed, Phys. Rev. Lett. 74, 4603 (1995).

[24] G. Isidori and P. Paradisi, Phys. Lett. B 639 (2006) 499 [arXiv:hep-ph/0605012]; W. S. Hou, Phys. Rev. D 48, 2342 (1993); A. G. Akeroyd and S. Recksiegel, J. Phys. G 29, 2311 (2003) [arXiv:hep-ph/0306037].

[25] G. Isidori and A. Retico, JHEP 0111, 001 (2001) [arXiv:hep-ph/0110121].

[26] A. Masiero, P. Paradisi and R. Petronzio, Phys. Rev. D 74 (2006) 011701 [arXiv:hep-ph/0511289].

[27] T. Alexopoulos et al. [KTeV Collaboration], Phys. Rev. D 70, 092006 (2004) [arXiv:hep-ex/0406002].

[28] A. Lai et al. [NA48 Collaboration], Phys. Lett. B 602, 41 (2004) [arXiv:hep-ex/0410059].

[29] L. Litov [NA48 Collaboration], arXiv:hep-ex/0501048.

[30] F. Ambrosino et al. [KLOE Collaboration], Phys. Lett. B 632, 43 (2006) [arXiv:hep-ex/0508027]. 
[31] F. Ambrosino et al. [KLOE Collaboration], Phys. Lett. B 626, 15 (2005) [arXiv:hep-ex/0507088].

[32] F. Ambrosino et al. [KLOE Collaboration], Phys. Lett. B 638, 140 (2006) [arXiv:hep-ex/0603041].

[33] A. Lai et al. [NA48 Collaboration], Phys. Lett. B 645, 26 (2007) [arXiv:hep-ex/0611052].

[34] F. Ambrosino et al. [KLOE Collaboration], Phys. Lett. B 636, 173 (2006) [arXiv:hep-ex/0601026].

[35] F. Ambrosino et al. [KLOE Collaboration], Eur. Phys. J. C 48, 767 (2006) [arXiv:hep-ex/0601025].

[36] J. R. Batley et al., Phys. Lett. B 653, 145 (2007).

[37] J. R. Batley et al. [NA48/2 Collaboration], Eur. Phys. J. C 50, 329 (2007) [arXiv:hep-ex/0702015].

[38] V. I. Romanovsky et al., arXiv:0704.2052 [hep-ex].

[39] F. Ambrosino et al. [KLOE Collaboration], arXiv:0707.2532 [hep-ex].

[40] F. Ambrosino et al. [KLOE Collaboration], Phys. Lett. B 632, 76 (2006) [arXiv:hep-ex/0509045].

[41] F. Ambrosino et al. [Kloe Collaboration], arXiv:0707.2654 [hep-ex].

[42] F. Ambrosino et al. [KLOE Collaboration], arXiv:0705.4408v3 [hep-ex].

[43] PDG, W.-M. Yao et al., J. Phys. G33 (2006) 1.

[44] L. Fiorini, PoS HEP2005, 288 (2006);

L. Fiorini, ph.D. thesis, Pisa (2005);

[45] V. Kozhuharov, KAON07 International Conference.

[46] F. Ambrosino et al. [Kloe Collaboration], arXiv:0707.4623v1 [hep-ex].

[47] E. Abouzaid et al., Phys. Rev. D 74, 097101 (2006).

[48] T. Alexopoulos et al. [KTeV Collaboration], Phys. Rev. D 70, 092007 (2004) [arXiv:hep-ex/0406003].

[49] F. Ambrosino et al. [KLOE Collaboration], Phys. Lett. B 636, 166 (2006) [arXiv:hep-ex/0601038].

[50] O. P. Yushchenko et al., Phys. Lett. B 589, 111 (2004) [arXiv:hep-ex/0404030].

[51] A. Lai et al. [NA48 Collaboration], Phys. Lett. B 604, 1 (2004) [arXiv:hep-ex/0410065].

[52] F. Ambrosino et al. [KLOE Collaboration], arXiv:0707.4631 [hep-ex].

[53] O. P. Yushchenko et al., Phys. Lett. B 581 (2004) 31.

[54] A. Lai et al. [NA48 Collaboration], Phys. Lett. B 647 (2007) 341.

[55] B. Ananthanarayan and B. Moussallam, JHEP 0406 (2004) 047 [arXiv:hep-ph/0405206]; J. F. Donoghue and A. F. Perez, Phys. Rev. D 55, 7075 (1997) [arXiv:hep-ph/9611331]; J. Bijnens and J. Prades, Nucl. Phys. B 490 (1997) 239 [arXiv:hep-ph/9610360].

[56] A. Kastner and H. Neufeld, work in progress. 
[57] H. Leutwyler and M. Roos, Z. Phys. C 25 (1984) 91.

[58] D. J. Antonio et al., arXiv:hep-lat/0702026;

[59] M. Okamoto [Fermilab Lattice, MILC and HPQCD Collaborations], Int. J. Mod. Phys. A 20, 3469 (2005);

[60] J. Portoles, arXiv:hep-ph/0703093; M. Jamin, J. A. Oller and A. Pich, JHEP 0402, 047 (2004); V. Cirigliano, G. Ecker, M. Eidemuller, R. Kaiser, A. Pich and J. Portoles, JHEP 0504, 006 (2005) [arXiv:hep-ph/0503108];

[61] A. Juttner, arXiv:0711.1239 [hep-lat];

[62] T. Kaneko, arXiv:0710.0698 [hep-ph].

[63] N. Tsutsui et al. [JLQCD Collaboration], PoS LAT2005, 357 (2006) [arXiv:hep-lat/0510068]; C. Dawson, T. Izubuchi, T. Kaneko, S. Sasaki and A. Soni, Phys. Rev. D 74, 114502 (2006) [arXiv:hep-ph/0607162]; D. Becirevic et al., Nucl. Phys. B 705, 339 (2005) [arXiv:hep-ph/0403217]; D. Brommel et al., arXiv:0710.2100 [hep-lat].

[64] C. Bernard et al., arXiv:0710.1118 [hep-lat].

[65] E. Follana, C. T. H. Davies, G. P. Lepage and J. Shigemitsu [HPQCD Collaboration], arXiv:0706.1726 [hep-lat].

[66] C. Allton et al. [RBC and UKQCD Collaborations], Phys. Rev. D 76, 014504 (2007) [arXiv:hep-lat/0701013].

[67] S. Aoki et al. [CP-PACS Collaboration], Phys. Rev. D 67, 034503 (2003) [arXiv:hep-lat/0206009]; A. Ali Khan et al. [CP-PACS Collaboration], Phys. Rev. D 65, 054505 (2002) [Erratum-ibid. D 67, 059901 (2003)] [arXiv:hep-lat/0105015]; S. Aoki et al. [JLQCD Collaboration], Phys. Rev. D 68, 054502 (2003) [arXiv:hep-lat/0212039]; Y. Aoki et al., Phys. Rev. D 72, 114505 (2005) [arXiv:hep-lat/0411006]; M. Gockeler et al., PoS LAT2006, 160 (2006) [arXiv:hep-lat/0610071]; C. Aubin et al. [MILC Collaboration], Phys. Rev. D 70, 114501 (2004) [arXiv:hep-lat/0407028];S. R. Beane, P. F. Bedaque, K. Orginos and M. J. Savage, Phys. Rev. D 75, 094501 (2007) [arXiv:hep-lat/0606023]; T. Ishikawa et al., PoS LAT2006, 181 (2006) [arXiv:hep-lat/0610050]; B. Blossier et al. [European Twisted Mass Collaboration], arXiv:0709.4574 [hep-lat].

[68] M. Creutz, "Why rooting fails", pleanary talk at Lattice 2007; A. Kronfeld, "Lattice QCD with Staggered Quarks: Why, Where, and How (Not)", pleanary talk at Lattice 2007.

[69] M. Hasenbusch, Phys. Lett. B 519, 177 (2001) [arXiv:hep-lat/0107019]; M. Luscher, Comput. Phys. Commun. 165, 199 (2005) [arXiv:hep-lat/0409106]; C. Urbach, K. Jansen, A. Shindler and U. Wenger, Comput. Phys. Commun. 174, 87 (2006) [arXiv:hep-lat/0506011]; T. Kaneko et al. [JLQCD Collaboration], PoS LAT2006, 054 (2006) [arXiv:hep-lat/0610036]; L. Del Debbio, L. Giusti, M. Luscher, R. Petronzio and N. Tantalo, JHEP 0602, 011 (2006) [arXiv:hep-lat/0512021]; T. Chiarappa et al., Eur. Phys. J. C 50, 373 (2007) [arXiv:hep-lat/0606011].

[70] D. Kadoh et al. [CS Collaboration], arXiv:0710.3467 [hep-lat]; N. Ukita et al. [CS Collaboration], arXiv:0710.3462 [hep-lat].

[71] S. Durr et al., arXiv:0710.4769 [hep-lat]; S. Durr et al., arXiv:0710.4866 [hep-lat].

[72] G. Furlan, F.G. Lannoy, C. Rossetti, and G. Segré, Nuovo Cim. 38 (1965) 1747. 
[73] J.C. Hardy and I.S. Towner, arXiv:0710.3181v1 [nucl-th].

[74] R. Barbieri et al., Phys. Lett. 156B (1985) 348; K. Hagiwara et al., Phys. Rev. Lett. 75 (1995) 3605; A. Kurylov and M. Ramsey-Musolf, Phys. Rev. Lett. 88 (2000) 071804.

[75] K. Ikado et al., Phys. Rev. Lett. 97, 251802 (2006) [arXiv:hep-ex/0604018]; B. Aubert et al. [BABAR Collaboration], Phys. Rev. D 76, 052002 (2007) [arXiv:0708.2260 [hep-ex]].

[76] K. G. Vosburgh Phys. et al., Rev. D 6, 1834, (1972).

[77] M. Adinolfi et al. [KLOE Collaboration], Phys. Lett. B 566, 61 (2003) [arXiv:hep-ex/0305035].

[78] A. Lai et al. [NA48 Collaboration], Phys. Lett. B 551, 7 (2003) [arXiv:hep-ex/0210053].

[79] E. J. Ramberg et al. [E731 Collaboration], Phys. Rev. Lett. 702525 (1993).

[80] A. Alavi-Harati et al. [KTeV Collaboration], Phys. Rev. Lett. 86761 (2001).

[81] E. Abouzaid et al. [KTeV Collaboration], Phys. Rev. D 74032004 (2006).

[82] G. D'Ambrosio, M. Miragliuolo, P. Sartorelli, in "DA $\Phi$ NE Physics Handbook", 231 (1992).

[83] V. P. Koptev et al., JETP Lett. 62, 877 (1995) [Pisma Zh. Eksp. Teor. Fiz. 61, 865 (1995)].

[84] R. J. Ott and T. W. Pritchard, Phys. Rev. D 3, 52 (1971).

[85] F. Lobkowicz, A. C. Melissinos, Y. Nagashima, S. Tewksbury, H. Von Briesen and J. D. Fox, Phys. Rev. 185, 1676 (1969).

[86] V. L. Fitch, C. A. Quarles, H. C. Wilkins, Phys. Rev. 140, 1088 (1965).

[87] T. Usher, M. Fero, M. Gee, N. A. Graf, M. Mandelkern, D. Schultz and J. Schultz, Phys. Rev. D 45, 3961 (1992).

[88] A. O. Weissenberg et al., Nucl. Phys. B 115, 55 (1975).

[89] L. B. Auerbach et al., Phys. Rev. 155, 1505 (1967).

[90] W. T. Ford et al., Phys. Rev. Lett. 251370 (1970).

[91] A. Sher et al., Phys. Rev. Lett. 91, 261802 (2003).

[92] P. T. Eschstruth et al., Phys. Rev. 165, 1487 (1968).

[93] R. Cesteret al., Phys. Lett. 21, 343 (1966).

[94] K. Horie et al. [KEK-E246 Collaboration], Phys. Lett. B 513, 311 (2001) [arXiv:hep-ex/0106006].

[95] J. Heintze et al., Phys. Lett. B 70, 482 (1977).

[96] D. R. Botterill et al., Phys. Rev. Lett. 21, 776 (1968).

[97] A. Aloisio et al. [KLOE Collaboration], Phys. Lett. B 597, 139 (2004) [arXiv:hep-ex/0307054].

[98] V. Bisi et al., Nuovo Cimento 35768 (1965).

[99] S. Pislak et al., Phys. Rev. D 67072004 (2003).

[100] H. Cramer, Mathematical Methods of Statistics, Princeton University Press, 1946, proves that this is the smallest possible error. 


\section{A. BRS fit procedure}

The fits to $K_{L}$ and $K^{ \pm}$data are performed with FORTRAN programs. MIGRAD is used for the minimization; errors are obtained with MINOs.

Suppose we have $N$ measurements of $M$ quantities, e.g., BRs, ratios of BRs, lifetimes, or partial widths, where $N \geq M$ as some quantities are measured by more than one experiment. Denote the $N$ measurements $x_{i}$, and the expected value for each as calculated from the free parameters of the fit $\bar{x}_{i}$. We also refer to the expected values for quantities measured by more than one experiment by the index $m$, i.e., $\bar{x}_{m}$ with $m=1, M$.

The errors on the input parameters are denoted $\sigma_{i}$. All errors on the input parameters are assumed to be Gaussian. For uncorrelated measurements with statistical and systematic errors quoted separately, we add the errors in quadrature. In many cases, the results for different quantities measured by the same experiment have correlated errors. The errors are then described by the covariance matrix $V_{i j}$, with $V_{i i}=\sigma_{i}^{2}$ and $V_{i j}=\rho_{i j} \sigma_{i} \sigma_{j}$. The expression to be minimized is then

$$
\chi^{2}=\sum_{i=1}^{N} \sum_{j=1}^{N}\left(x_{i}-\bar{x}_{i}\right)\left(x_{j}-\bar{x}_{j}\right)(\mathbf{V})_{i j}^{-1} .
$$

In practice, $\mathbf{V}$ is block diagonal and only the relevant sub-matrices are inverted.

The penalty method is used to implement the constraint on the sum of the BRs. In this method, a term $G\left(1-\sum \mathrm{BR}\right)^{2}$ is added to the $\chi^{2}$ to be minimized. As $G$ is increased, the constraint is enforced with greater and greater precision and the result of the fit saturates (until at some very large value of $G$, problems related to the precision of the calculation set in). $G$ is determined by trial and error; its value is $2 \times 10^{7}$ for the $K_{L}$ fit and $1 \times 10^{8}$ for the $K^{ \pm}$fit. The $K_{L}$ fit is somewhat more sensitive to the value of $G$, because the $K_{L}$ BRs entering the fit span three orders of magnitude. As a result, precision problems have a greater effect on the constraint balance.

Once the fit has been performed, scale factors are calculated and used as described in the general introduction to the PDG compilation. As above, our $N$ data points consist of $m=1, M$ distinct measured quantities, each of which is measured by $n_{m}$ experiments, indexed by $k_{m}$. $\left(N=\sum_{m} n_{m}\right)$. Here it is useful to adopt the notation $x_{m k_{m}} \pm \sigma_{m k_{m}}$ for the individual measurements, and the notation $\bar{x}_{m}$ for the expected value for the $m^{\text {th }}$ quantity. After the fit is performed once, the error $\bar{\sigma}_{m}$ on $\bar{x}_{m}$ is evaluated from the output covariance matrix for the free fit parameters. Then, the scale factor for the measured quantity $m$ is calculated as

$$
S_{m}^{2}=\frac{1}{n_{m}} \sum_{k_{m}=1}^{n_{m}} \frac{\left(x_{m k_{m}}-\bar{x}_{m}\right)^{2}}{\sigma_{m k_{m}}^{2}-\bar{\sigma}_{m}^{2}} .
$$

Next, the errors $\sigma_{m k_{m}}$ are scaled by the greater value of $S_{m}$ and unity. For subsets of correlated measurements (all from the same experiment), the index $k_{m}$ can be omitted to write $V_{m m^{\prime}}=\rho_{m m^{\prime}} \sigma_{m} \sigma_{m^{\prime}}$; the scale factors are applied to $\sigma_{m}$ and $\sigma_{m^{\prime}}$ and $\mathbf{V}$ and its inverse are recalculated. Finally, the fit is performed a second time. For each of the fit parameters, we report the central value from the first fit, and the error (and correlations) 
from the second fit. The scale factors for the errors on the fit parameters are defined as the ratios of the errors from the second fit to those from the first. The value of $\chi^{2}$ reported is from the first fit.

For the purposes of comparison, pull values are calculated for each measurement simply as $\left(x_{i}-\bar{x}_{i}\right) / \sigma_{i}$.

For the BR/lifetime fits, the errors are in general symmetric to within rounding error; in any case we report the greater of the positive and negative MINOS errors.

\section{B. Fit for $K_{L}$ BRs and lifetime}

The 8 free parameters in the $K_{L}$ fit are $\mathrm{BR}\left(K_{e 3}\right), \mathrm{BR}\left(K_{\mu 3}\right), \operatorname{BR}\left(3 \pi^{0}\right), \operatorname{BR}\left(\pi^{+} \pi^{-} \pi^{0}\right)$, $\operatorname{BR}\left(\pi^{+} \pi^{-}\right), \operatorname{BR}\left(\pi^{0} \pi^{0}\right), \operatorname{BR}(\gamma \gamma)$, and $\tau_{K_{L}}$. The fit makes use of the 18 measurements in Table 11. With one constraint, the fit has 11 degrees of freedom.

The differences between our fit and the 2006 PDG fit are as follows:

- In our fit, the intermediate $\mathrm{KTeV}$ and $\mathrm{KLOE}$ values (i.e., before applying constraints) are the inputs, and the complete error matrix is used to handle the correlations between the measurements from each experiment. In the 2006 PDG fit, the final $\mathrm{KTeV}$ and KLOE BR results were used and one measurement involving $\operatorname{BR}\left(3 \pi^{0}\right)$ was removed in each case.

- Our fit makes use of the preliminary $\mathrm{BR}\left(3 \pi^{0}\right)[29]$ and new $\mathrm{BR}\left(\pi^{+} \pi^{-}\right) / \mathrm{BR}\left(K_{e 3}\right)$ [33] measurements from NA48.

- Our fit parameter $\mathrm{BR}\left(\pi^{+} \pi^{-}\right)$is understood to be inclusive of the DE component. This helps to satisfy the constraint. The input data are treated consistently in this respect.

- We do not make use of the measurement of $\operatorname{BR}(\gamma \gamma) / \mathrm{BR}\left(\pi^{0} \pi^{0}\right)$ from NA31 (Burkhardt '87), since both we and the PDG have excluded the other measurements from NA31.

\section{Notes on data in Table 11}

1. Direct measurement of $\tau_{K_{L}}$ from $3 \pi^{0}$ events; independent of other KLOE measurements 31].

2. We make use of the KLOE results for the main $K_{L}$ BRs $(\# 3$, \#4, \#6, and \#9) obtained before applying constraints on the sum of the BRs [30]. The BR values in Table 11 thus depend on $\tau_{K_{L}}$ as follows:

$$
\mathrm{BR}=\frac{\mathrm{BR}^{0}}{1+0.0128 \mathrm{~ns}^{-1}\left(\tau_{K_{L}}^{0}-\tau_{K_{L}}\right)},
$$

where $\tau_{K_{L}}^{0}=51.54 \mathrm{~ns}$. The errors listed for these values in Ref. [30] include an explicit contribution from the uncertainty on the reference value of $\tau_{K_{L}}$. This contribution has been unfolded from the errors in Table 11. In addition, these four BR measurements 


\begin{tabular}{|c|c|c|c|c|}
\hline Point & Parameter & Value & Source & Note \\
\hline 1 & $\tau_{K_{L}}$ & $50.92(30) \mathrm{ns}$ & KLOE '05 & 131 \\
\hline 2 & $\tau_{K_{L}}$ & $51.54(44) \mathrm{ns}$ & Vosburgh '72 & 76 \\
\hline 3 & $\mathrm{BR}\left(K_{e 3}\right)$ & $0.4049(21)$ & KLOE '06 & 230 \\
\hline 4 & $\operatorname{BR}\left(K_{\mu 3}\right)$ & $0.2726(16)$ & KLOE '06 & 230 \\
\hline 5 & $\operatorname{BR}\left(K_{\mu 3}\right) / \operatorname{BR}\left(K_{e 3}\right)$ & $0.6640(26)$ & $\mathrm{KTeV}$ '04 & 3 27 \\
\hline 6 & $\operatorname{BR}\left(3 \pi^{0}\right)$ & $0.2018(24)$ & KLOE '06 & 230 \\
\hline 7 & $\operatorname{BR}\left(3 \pi^{0}\right) / \tau_{K_{L}}$ & 3.795(58) MHz & NA48 '04 & 429 \\
\hline 8 & $\operatorname{BR}\left(3 \pi^{0}\right) / \mathrm{BR}\left(K_{e 3}\right)$ & $0.4782(55)$ & $\mathrm{KTeV}$ '04 & 3 27 \\
\hline 9 & $\mathrm{BR}\left(\pi^{+} \pi^{-} \pi^{0}\right)$ & $0.1276(15)$ & KLOE '06 & 230 \\
\hline 10 & $\operatorname{BR}\left(\pi^{+} \pi^{-} \pi^{0}\right) / \operatorname{BR}\left(K_{e 3}\right)$ & $0.3078(18)$ & $\mathrm{KTeV}$ '04 & 3 27 \\
\hline 11 & $\operatorname{BR}\left(\pi^{+} \pi^{-}\right) / \operatorname{BR}\left(K_{e 3}\right)$ & $0.004856(29)$ & KTeV '04 & 3,5 27 \\
\hline 12 & $\operatorname{BR}\left(\pi^{+} \pi^{-}\right) / \operatorname{BR}\left(K_{e 3}\right)$ & $0.004826(27)$ & NA48 '06 & 533 \\
\hline 13 & $\operatorname{BR}\left(\pi^{+} \pi^{-}\right) / \operatorname{BR}\left(K_{\mu 3}\right)$ & $0.007275(68)$ & KLOE '06 & 532 \\
\hline 14 & $\mathrm{BR}\left(K_{e 3}\right) / \mathrm{BR}(2$ tracks $)$ & $0.4978(35)$ & NA48 '04 & 628 \\
\hline 15 & $\operatorname{BR}\left(\pi^{0} \pi^{0}\right) / \operatorname{BR}\left(3 \pi^{0}\right)$ & $0.004446(25)$ & KTeV '04 & 3 27 \\
\hline 16 & $\mathrm{BR}\left(\pi^{0} \pi^{0}\right) / \mathrm{BR}\left(\pi^{+} \pi^{-}\right)$ & $0.4391(13)$ & PDG '06 & 743 \\
\hline 17 & $\operatorname{BR}(\gamma \gamma) / \operatorname{BR}\left(3 \pi^{0}\right)$ & $0.00279(3)$ & KLOE '03 & 77 \\
\hline 18 & $\operatorname{BR}(\gamma \gamma) / \operatorname{BR}\left(3 \pi^{0}\right)$ & $0.00281(2)$ & NA48 '03 & 78 \\
\hline
\end{tabular}

Table 11: Input data used for the fit to $K_{L}$ BRs and lifetime.

are correlated by common systematics as described in KLOE Note 204, although the full correlation matrix is not given therein. The correlation matrix is as follows:

$\begin{array}{ccccc} & 3 & 4 & 6 & 9 \\ 3 & 1.000 & 0.091 & 0.069 & 0.494 \\ 4 & & 1.000 & -0.025 & 0.267 \\ 6 & & & 1.000 & 0.074 \\ 9 & & & & 1.000\end{array}$

3. The correlation matrix for the $\mathrm{KTeV}$ relative $\mathrm{BR}$ measurements (\#5, \#8, \#10, \#11, and \#15) is as follows [27]:

$\begin{array}{cccccc} & 5 & 8 & 10 & 11 & 15 \\ 5 & 1.00 & 0.14 & 0.21 & 0.24 & 0.09 \\ 8 & & 1.00 & -0.06 & -0.07 & 0.30 \\ 10 & & & 1.00 & 0.49 & 0.04 \\ 11 & & & & 1.00 & 0.07 \\ 15 & & & & & 1.00\end{array}$

4. This is based on the preliminary NA48 measurement $\mathrm{BR}\left(3 \pi^{0}\right)=0.1966(34)$, as reported in Ref. [29]. R. Wanke has confirmed that the 2004 PDG value for $\tau_{K_{L}}$ was 
used to obtain this result. The NA48 value for this BR scales directly with the lifetime value used. R. Wanke has supplied the value in the table for the partial width, with the contribution to the error on the BR from the uncertainty on the $K_{L}$ lifetime unfolded.

5. The fit value of $\mathrm{BR}\left(\pi^{+} \pi^{-}\right)$includes the DE component. The KLOE measurement of $\mathrm{BR}\left(\pi^{+} \pi^{-}\right) / \mathrm{BR}\left(K_{\mu 3}\right)$ 32 (\#13) is inclusive of DE. The KTeV and NA48 measurements of $\operatorname{BR}\left(\pi^{+} \pi^{-}\right) / \operatorname{BR}\left(K_{e 3}\right)$ (\#11 and \#12, respectively) are treated as follows.

- We use the average values of DE/(DE + IB) from Refs. [79, 80] and [81] together with $\operatorname{BR}\left(\pi^{+} \pi^{-} \gamma_{\mathrm{IB}}, E_{\gamma}>20 \mathrm{MeV}\right) / \mathrm{BR}\left(\pi^{+} \pi^{-}\right)=7.00 \times 10^{-3}$ [82, to calculate that $\mathrm{DE}$ accounts for $1.52(7) \%$ of the inclusive $K_{L} \rightarrow \pi^{+} \pi^{-}$width. The error on this correction is negligible for the purposes of the fit.

- The $\mathrm{KTeV}$ measurement of $\operatorname{BR}\left(\pi^{+} \pi^{-}\right) / \mathrm{BR}\left(K_{e 3}\right)$ (\#11) excludes $\mathrm{DE}$ (in the sense that Ref. [27] says that DE is not in the generator for the acceptance calculation). We therefore subtract $1.52 \%$ from the fit value of the ratio when calculating the contribution to $\chi^{2}$ from this $\mathrm{KTeV}$ measurement.

- The contribution from DE to the NA48 measurement $\operatorname{BR}\left(\pi^{+} \pi^{-}\right) / \mathrm{BR}\left(K_{e 3}\right)=$ $4.835(22)(20) \times 10^{-3}$ is estimated to be $0.19(1) \%$, which we subtract to obtain value \#12. We then handle the data point in the same way as we do for $\mathrm{KTeV}$.

6. For our fit, $\mathrm{BR}(2$ tracks $)$ has to be calculated from the free fit parameters. Like the PDG, we use

$$
\begin{aligned}
\mathrm{BR}(2 \text { tracks })= & \operatorname{BR}\left(K_{e 3}\right)+\operatorname{BR}\left(K_{\mu 3}\right)+0.03508 \operatorname{BR}\left(3 \pi^{0}\right) \\
& +\operatorname{BR}\left(\pi^{+} \pi^{-} \pi^{0}\right)+\operatorname{BR}\left(\pi^{+} \pi^{-}\right) .
\end{aligned}
$$

7. From the ETAFIT analysis 43.

\section{B.1 Results}

The results of the fit are summarized in Table 12. The output correlation matrix is given in Table 13. The pull values for the input measurements are listed in Table 14. With respect to the 2006 PDG fit, our fit has a somewhat lower $\chi^{2}$ probability.

When our fit is run without inclusion of points \#7 and \#12, without DE corrections for the $\pi^{+} \pi^{-}$channel, and with the measurement of $\operatorname{BR}(\gamma \gamma) / \operatorname{BR}\left(\pi^{0} \pi^{0}\right)$ from NA31, we reproduce the $2006 \mathrm{PDG}$ fit result. In this configuration, the only difference between our fit and the 2006 PDG fit is the treatment of the BR and lifetime data from KLOE and $\mathrm{KTeV}$. We obtain the same values for all 8 fit parameters, with $\chi^{2} / \mathrm{ndf}=14.9 / 10$. Our scale factors in this case are more uniform than those obtained in the 2006 PDG fit; in particular, for $\operatorname{BR}\left(K_{e 3}\right), \operatorname{BR}\left(3 \pi^{0}\right)$, and $\operatorname{BR}\left(\pi^{+} \pi^{-} \pi^{0}\right)$ we have $S=1.2,1.1$, and 1.4, to be compared with the second column of Table 12.

Excluding the measurement of $\operatorname{BR}(\gamma \gamma) / \operatorname{BR}\left(\pi^{0} \pi^{0}\right)$ from NA31 has a negligible effect on the fit results, while the number of degrees of freedom is reduced by one, giving $\chi^{2} / \mathrm{ndf}=$ $14.9 / 9(9.4 \%)$. Turning on the DE correction degrades the fit quality from $\chi^{2} / \mathrm{ndf}=14.9 / 9$ 


\begin{tabular}{lcccc}
\hline \hline & \multicolumn{2}{c}{ This fit } & \multicolumn{2}{c}{ 2006 PDG } \\
& \multicolumn{1}{c}{18 measurements } & \multicolumn{2}{c}{17 measurements } \\
& $\chi^{2} / \mathrm{ndf}=19.7 / 11$ & $(4.9 \%)$ & $\chi^{2} / \mathrm{ndf}=14.8 / 10$ & $(14.0 \%)$ \\
\hline Parameter & Result & $S$ & Result & $S$ \\
\hline $\operatorname{BR}\left(K_{e 3}\right)$ & $0.4058(9)$ & 1.3 & $0.4053(15)$ & 2.1 \\
$\operatorname{BR}\left(K_{\mu 3}\right)$ & $0.2706(8)$ & 1.3 & $0.2702(7)$ & 1.0 \\
$\operatorname{BR}\left(3 \pi^{0}\right)$ & $0.1943(10)$ & 1.3 & $0.1956(14)$ & 1.9 \\
$\operatorname{BR}\left(\pi^{+} \pi^{-} \pi^{0}\right)$ & $0.1259(8)$ & 1.5 & $0.1256(5)$ & 1.0 \\
$\operatorname{BR}\left(\pi^{+} \pi^{-}\right)$ & $1.986(7) \times 10^{-3}$ & 1.2 & $1.976(8) \times 10^{-3}$ & 1.0 \\
$\operatorname{BR}\left(\pi^{0} \pi^{0}\right)$ & $8.60(5) \times 10^{-4}$ & 1.5 & $8.69(4) \times 10^{-4}$ & 1.1 \\
$\operatorname{BR}(\gamma \gamma)$ & $5.45(4) \times 10^{-4}$ & 1.1 & $5.48(5) \times 10^{-4}$ & 1.2 \\
$\operatorname{BR}\left(\tau_{K_{L}}\right)$ & $51.15(20) \mathrm{ns}$ & 1.1 & $51.14(21) \mathrm{ns}$ & 1.0 \\
\hline \hline
\end{tabular}

Table 12: Results of fit to $K_{L}$ BRs and lifetime, with comparison to 2006 PDG fit.

$$
\begin{aligned}
& \begin{array}{llllllll}
+1.000 & -0.286 & -0.422 & -0.288 & +0.112 & -0.282 & -0.270 & -0.005
\end{array} \\
& \begin{array}{lllllll}
+1.000 & -0.378 & -0.217 & -0.046 & -0.216 & -0.241 & +0.183
\end{array} \\
& \begin{array}{llllll}
+1.000 & -0.354 & -0.029 & +0.609 & +0.637 & -0.036
\end{array} \\
& \begin{array}{lllll}
+1.000 & -0.035 & -0.205 & -0.226 & -0.127
\end{array} \\
& +1.000 \quad+0.205 \quad-0.020-0.033 \\
& +1.000+0.387-0.029 \\
& +1.000-0.027 \\
& +1.000
\end{aligned}
$$

Table 13: Correlation matrix for output parameters of $K_{L}$ fit.

to 19.6/9 (2.02\%). When points \#7 and \#12 are added, the fit quality is slightly improved and the result in Table 12 is obtained.

However, the fit quality improves dramatically when the PDG ETAFIT result for $\operatorname{BR}\left(\pi^{0} \pi^{0}\right) / \operatorname{BR}\left(\pi^{+} \pi^{-}\right)(\# 16)$ is removed. This is true independently of whether or not the DE correction and/or the additional results are included. For example, our same fit with the PDG ETAFIT point removed gives $\chi^{2} / \mathrm{ndf}=14.8 / 10(13.9 \%)$, with changes in the fit values for the BRs at the $1 \sigma$ level. In all other configurations (DE correction on/off; points \#7, \#12, NA31 $\operatorname{BR}(\gamma \gamma) / \operatorname{BR}\left(\pi^{0} \pi^{0}\right)$ included/excluded), the fit gives similar results.

Using the values of $\operatorname{BR}\left(\pi^{+} \pi^{-}\right)$and $\operatorname{BR}\left(\pi^{0} \pi^{0}\right)$ from our fits including and excluding the PDG ETAFIT point, we have evaluated $\operatorname{Re} \epsilon^{\prime} / \epsilon$ from

$$
\operatorname{Re} \epsilon^{\prime} / \epsilon=\frac{1}{6}\left[1-R_{S} \frac{\operatorname{BR}\left(\pi^{0} \pi^{0}\right)}{\operatorname{BR}\left(\pi^{+} \pi^{-}\right)}\right]
$$

with $R_{S} \equiv \operatorname{BR}\left(K_{S} \rightarrow \pi^{+} \pi^{-}\right) / \operatorname{BR}\left(K_{S} \rightarrow \pi^{0} \pi^{0}\right)=2.2549(54)$ [35] as described in Sec. 3.2. We obtain

$$
\begin{aligned}
& \operatorname{Re} \epsilon^{\prime} / \epsilon=(-25 \pm 23) \times 10^{-4} \quad \text { (without ETAFIT); } \\
& \operatorname{Re} \epsilon^{\prime} / \epsilon=(14 \pm 11) \times 10^{-4} \quad \text { (with ETAFIT); }
\end{aligned}
$$




\begin{tabular}{|c|c|c|c|}
\hline Point & Parameter & Source & Pull \\
\hline 6 & $\operatorname{BR}\left(3 \pi^{0}\right)$ & KLOE '06 & +2.74 \\
\hline 2 & $\tau_{K_{L}}$ & Vosburgh '72 & +0.88 \\
\hline 15 & $\operatorname{BR}\left(\pi^{0} \pi^{0}\right) / \operatorname{BR}\left(3 \pi^{0}\right)$ & KTeV '04 & +0.81 \\
\hline 9 & $\mathrm{BR}\left(\pi^{+} \pi^{-} \pi^{0}\right)$ & KLOE '06 & +0.71 \\
\hline 4 & $\operatorname{BR}\left(K_{\mu 3}\right)$ & KLOE '06 & +0.41 \\
\hline 18 & $\operatorname{BR}(\gamma \gamma) / \operatorname{BR}\left(3 \pi^{0}\right)$ & NA48 '03 & +0.31 \\
\hline 12 & $\mathrm{BR}\left(\pi^{+} \pi^{-}\right) / \mathrm{BR}\left(K_{e 3}\right)$ & NA48'06 & +0.22 \\
\hline 7 & $\operatorname{BR}\left(3 \pi^{0}\right) / \tau K_{L}$ & NA48'04 & -0.07 \\
\hline 8 & $\operatorname{BR}\left(3 \pi^{0}\right) / \operatorname{BR}\left(K_{e 3}\right)$ & $\mathrm{KTeV} ' 04$ & -0.13 \\
\hline 17 & $\operatorname{BR}(\gamma \gamma) / \operatorname{BR}\left(3 \pi^{0}\right)$ & KLOE '03 & -0.46 \\
\hline 16 & $\operatorname{BR}\left(\pi^{0} \pi^{0}\right) / \operatorname{BR}\left(\pi^{+} \pi^{-}\right)$ & PDG '06 & -0.57 \\
\hline 14 & $\mathrm{BR}\left(K_{e 3}\right) / \mathrm{BR}(2$ tracks $)$ & NA48 '04 & -0.71 \\
\hline 1 & $\tau_{K_{L}}$ & KLOE '05 & -0.78 \\
\hline 13 & $\operatorname{BR}\left(\pi^{+} \pi^{-}\right) / \operatorname{BR}\left(K_{\mu 3}\right)$ & KLOE '06 & -0.94 \\
\hline 5 & $\operatorname{BR}\left(K_{\mu 3}\right) / \operatorname{BR}\left(K_{e 3}\right)$ & KTeV'04 & -1.11 \\
\hline 11 & $\operatorname{BR}\left(\pi^{+} \pi^{-}\right) / \operatorname{BR}\left(K_{e 3}\right)$ & KTeV'04 & -1.32 \\
\hline 3 & $\operatorname{BR}\left(K_{e 3}\right)$ & KLOE '06 & -1.37 \\
\hline 10 & $\operatorname{BR}\left(\pi^{+} \pi^{-} \pi^{0}\right) / \operatorname{BR}\left(K_{e 3}\right)$ & KTeV '04 & -1.39 \\
\hline
\end{tabular}

Table 14: Pull values for input data used in fit to $K_{L}$ BRs and lifetime.

to be compared to the current PDG average, $(16.7 \pm 2.3) \times 10^{-4}$. The ETAFIT point is very precise; when it is included, the fit results for $\operatorname{BR}\left(\pi^{0} \pi^{0}\right) / \mathrm{BR}\left(\pi^{+} \pi^{-}\right)$are highly constrained. This pulls down the value of $\operatorname{BR}\left(\pi^{0} \pi^{0}\right)$, and, also of $\mathrm{BR}\left(3 \pi^{0}\right)$, via the $\mathrm{KTeV}$ measurement of $\operatorname{BR}\left(\pi^{0} \pi^{0}\right) / \operatorname{BR}\left(3 \pi^{0}\right)$. As seen from Table 14, the measurement with the largest positive pull on the fit is the KLOE measurement of $\mathrm{BR}\left(3 \pi^{0}\right)$, which PDG has chosen to eliminate from the 2006 fit as part of their treatment of the correlated KLOE measurements.

We emphasize that the values of $\mathrm{BR}\left(K_{e 3}\right)$ and $\mathrm{BR}\left(K_{\mu 3}\right)$ are not affected very much by these developments. The scale factor, and hence the reported error, on $\mathrm{BR}\left(K_{e 3}\right)$ is significantly smaller in our fit, which spreads the pulls somewhat more evenly over the different measurements than does the PDG fit.

\section{Fit for $K^{ \pm}$BRs and lifetime}

The 7 free parameters in the $K^{ \pm}$fit are $\mathrm{BR}\left(K_{\mu 2}\right), \mathrm{BR}\left(\pi \pi^{0}\right), \operatorname{BR}(\pi \pi \pi), \operatorname{BR}\left(K_{e 3}\right), \operatorname{BR}\left(K_{\mu 3}\right)$, $\operatorname{BR}\left(\pi \pi^{0} \pi^{0}\right)$, and $\tau_{K^{ \pm}}$. The fit makes use of the 26 measurements in Table 15. With one constraint, the fit has 24 degrees of freedom.

The principal difference between the fit performed here and the 2006 PDG fit is that our fit includes the following recent measurements:

- Preliminary $\tau_{K^{ \pm}}$from KLOE $(\# 6, \# 7)$;

- Preliminary $\operatorname{BR}\left(K_{e 3}\right)$ and $\operatorname{BR}\left(K_{\mu 3}\right)$ from $\operatorname{KLOE}(\# 14, \# 20)$; 


\begin{tabular}{|c|c|c|c|c|}
\hline Point & Parameter & Value & Source & Note \\
\hline 1 & $\tau_{K^{ \pm}}$ & $12.451(30) \mathrm{ns}$ & Koptev '95 [83] & 1 \\
\hline 2 & $\tau_{K^{ \pm}}$ & $12.368(41) \mathrm{ns}$ & Koptev '95 83 & 1 \\
\hline 3 & $\tau_{K^{ \pm}}$ & $12.380(16) \mathrm{ns}$ & Ott '71 84 & \\
\hline 4 & $\tau_{K^{ \pm}}$ & $12.272(36) \mathrm{ns}$ & Lobkowicz '69 85 & \\
\hline 5 & $\tau_{K^{ \pm}}$ & $12.443(38) \mathrm{ns}$ & Fitch '65 86 & \\
\hline 6 & $\tau_{K^{ \pm}}$ & $12.367(78) \mathrm{ns}$ & KLOE '06 42 & 2 \\
\hline 7 & $\tau_{K^{ \pm}}$ & 12.391(55) ns & KLOE '07 42 & 2 \\
\hline 8 & $\operatorname{BR}\left(K_{\mu 2}\right)$ & $0.6366(17)$ & KLOE '06 40 & \\
\hline 9 & $\operatorname{BR}\left(\pi \pi^{0}\right)$ & $0.2066(11)$ & KLOE '07 41 & \\
\hline 10 & $\operatorname{BR}\left(\pi \pi^{0}\right) / \operatorname{BR}\left(K_{\mu 2}\right)$ & $0.3329(48)$ & Usher '92 87] & \\
\hline 11 & $\operatorname{BR}\left(\pi \pi^{0}\right) / \operatorname{BR}\left(K_{\mu 2}\right)$ & $0.3355(57)$ & Weissenberg '76 88] & \\
\hline 12 & $\operatorname{BR}\left(\pi \pi^{0}\right) / \operatorname{BR}\left(K_{\mu 2}\right)$ & $0.3277(65)$ & Auerbach '67 89 & \\
\hline 13 & $\Gamma(\pi \pi \pi)$ & 4.513(24) MHz & Ford '70 90 & \\
\hline 14 & $\operatorname{BR}\left(K_{e 3}\right)$ & $0.04965(53)$ & KLOE '07 39 & 2,4 \\
\hline 15 & $\operatorname{BR}\left(K_{e 3}\right) / \operatorname{BR}\left(\pi \pi^{0}+K_{\mu 3}+\pi 2 \pi^{0}\right)$ & $0.1962(36)$ & Sher '03 91 & \\
\hline 16 & $\operatorname{BR}\left(K_{e 3}\right) / \operatorname{BR}\left(K_{\mu 2}+\pi \pi^{0}\right)$ & $0.0616(22)$ & Eschstruth '68 [92] & \\
\hline 17 & $\operatorname{BR}\left(K_{e 3}\right) / \operatorname{BR}\left(K_{\mu 2}+\pi \pi^{0}\right)$ & $0.0589(21)$ & Cester '66[93] & \\
\hline 18 & $\operatorname{BR}\left(K_{e 3}\right) / \operatorname{BR}\left(\pi \pi^{0}\right)$ & $0.2449(16)$ & ISTRA '07 38 & 2 \\
\hline 19 & $\operatorname{BR}\left(K_{e 3}\right) / \mathrm{BR}\left(\pi \pi^{0}\right)$ & $0.2470(10)$ & NA48 '07 37 & 5 \\
\hline 20 & $\operatorname{BR}\left(K_{\mu 3}\right)$ & $0.03233(39)$ & KLOE '07 39 & 2,4 \\
\hline 21 & $\operatorname{BR}\left(K_{\mu 3}\right) / \operatorname{BR}\left(\pi \pi^{0}\right)$ & $0.1636(7)$ & NA48 '07 37 & 5 \\
\hline 22 & $\operatorname{BR}\left(K_{\mu 3}\right) / \operatorname{BR}\left(K_{e 3}\right)$ & $0.671(11)$ & Horie '01 94] & \\
\hline 23 & $\operatorname{BR}\left(K_{\mu 3}\right) / \operatorname{BR}\left(K_{e 3}\right)$ & $0.670(14)$ & Heintze '77 [95] & \\
\hline 24 & $\operatorname{BR}\left(K_{\mu 3}\right) / \operatorname{BR}\left(K_{e 3}\right)$ & $0.667(17)$ & Botterill '68 96 & \\
\hline 25 & $\operatorname{BR}\left(\pi \pi^{0} \pi^{0}\right)$ & $0.01763(26)$ & KLOE '04 97 & \\
\hline 26 & $\operatorname{BR}\left(\pi \pi^{0} \pi^{0}\right) / \operatorname{BR}(\pi \pi \pi)$ & $0.303(9)$ & Bisi '65 98 & \\
\hline
\end{tabular}

Table 15: Input data used for the fit to $K^{ \pm}$BRs and lifetime.

- Preliminary $\operatorname{BR}\left(\pi \pi^{0}\right)$ from $\operatorname{KLOE}(\# 9)$;

- Preliminary $\mathrm{BR}\left(K_{e 3}\right) / \mathrm{BR}\left(\pi \pi^{0}\right)$ from ISTRA+ (\#18);

- $\operatorname{BR}\left(K_{e 3}\right) / \operatorname{BR}\left(\pi \pi^{0}\right)(\# 19)$ and $\operatorname{BR}\left(K_{\mu 3}\right) / \operatorname{BR}\left(\pi \pi^{0}\right)$ from NA48/2 (\#21).

These new measurements have a profound impact on the results of the fit. Other differences are as follows.

- In the $2006 \mathrm{PDG}$ fit, $\mathrm{BR}\left(\pi^{0} \pi^{0} e \nu\right)$ is a free parameter (but curiously, $\mathrm{BR}(\pi \pi e \nu)$, for which there is a published measurement from E865 with much higher accuracy [99], is not). The PDG fit therefore uses three measurements involving $\operatorname{BR}\left(\pi^{0} \pi^{0} e \nu\right)$ and $\operatorname{BR}\left(\pi^{0} \pi^{0} e \nu\right) / \operatorname{BR}\left(K_{e 3}\right)$ that are not used in our fit. 
- We don't use the six BR measurements from Chiang '72. Our reading of Chiang '72 suggests that no attempt was made to implement radiative corrections for the branching ratio analysis. In addition, the six BR measurements from Chiang '72 are constrained to sum to unity. The correlation matrix is not available. PDG omits $\operatorname{BR}(\pi \pi \pi)$.

It would be highly desirable to discard many other old measurements in the $K^{ \pm}$fit as 2006 PDG has done for the $K_{L}$ fit. Unfortunately, are no recent measurements involving $\operatorname{BR}(\pi \pi \pi)$. As a result, the fit is unstable if only recent measurements are used.

\section{Notes on data in Table 15}

1. The only difference between the Koptev measurements is the material used for the kaon stopper (\#1-U, \#2-Cu).

2. Preliminary measurement.

3. The dependence of these BRs on the $K^{ \pm}$lifetime is accounted for in the fit:

$$
\mathrm{BR}=\mathrm{BR}^{0}\left[1+0.0405\left(\tau_{K^{ \pm}}-\tau_{K^{ \pm}}^{0}\right)\right]
$$

where $\mathrm{BR}^{0}$ is evaluated with $\tau_{K^{ \pm}}=12.360 \mathrm{~ns}$. The uncertainty from the value of $\tau_{K^{ \pm}}$may not have been properly unfolded. In addition, these two measurements are have a correlation coefficient of 0.627 , mainly from the use of common efficiency corrections.

4. The recent NA48 publication 37] gives values for $\operatorname{BR}\left(K_{e 3}\right) / \operatorname{BR}\left(\pi \pi^{0}\right)$, $\mathrm{BR}\left(K_{\mu 3}\right) / \mathrm{BR}\left(\pi \pi^{0}\right)$. The value of $\mathrm{BR}\left(K_{e 3}\right) / \mathrm{BR}\left(\pi \pi^{0}\right)$ has been updated at KAON07.

\section{C.1 Results}

The results of the fit are summarized in Table 16. The output correlation matrix is given in Table 17. The pull values for the input measurements are listed in Table 18. The poor fit quality derives from the following sources.

- The fit quality is significantly degraded by the scatter in the five older measurements of $\tau_{K^{ \pm}}$; when these are replaced with their PDG average with scaled error, $\tau_{K^{ \pm}}=$ $12.385(25) \mathrm{ns}$, the fit gives $\chi^{2} / \mathrm{ndf}=24.3 / 16(8.4 \%)$, with no significant changes in the results. Note that after this treatment the fit quality is about the same as it is for the 2006 PDG fit (which, however, includes all of the older $\tau_{K^{ \pm}}$measurements without taking the average).

- There is some conflict among the newer measurements involving $\mathrm{BR}\left(K_{e 3}\right)$, as seen from the pulls for the NA48 '07 (\#19), Sher '03 (\#15), ISTRA '07 (\#20), and KLOE '07 (\#14) measurements: $+1.04,-0.26,-0.74$, and -2.13 , respectively.(Table 18).

The evolution of the average values of the BRs for $K_{\ell 3}^{ \pm}$decays and for the important normalization channels as a result of the introduction of the preliminary measurements is evident in Fig. 2. The figure dramatically illustrates why experiments that measure ratios such as $\mathrm{BR}\left(K_{e 3}\right) / \mathrm{BR}\left(\pi \pi^{0}\right)$ should always quote the ratio with usable errors, in addition to the normalized, final value. 


\begin{tabular}{|c|c|c|c|c|}
\hline \multirow[b]{2}{*}{ Parameter } & \multicolumn{2}{|c|}{$\begin{array}{c}\text { This fit } \\
26 \text { measurements } \\
\chi^{2} / \text { ndf }=42 / 20(0.31 \%)\end{array}$} & \multicolumn{2}{|c|}{$\begin{array}{c}2006 \text { PDG } \\
26 \text { measurements } \\
\chi^{2} / \text { ndf }=30 / 19(5.2 \%)\end{array}$} \\
\hline & Result & $S$ & Result & $S$ \\
\hline $\operatorname{BR}\left(K_{\mu 2}\right)$ & $63.57(11) \%$ & 1.1 & $63.44(14) \%$ & 1.2 \\
\hline $\operatorname{BR}\left(\pi \pi^{0}\right)$ & $20.64(8) \%$ & 1.1 & $20.92(12) \%$ & 1.1 \\
\hline $\operatorname{BR}(\pi \pi \pi)$ & $5.595(31) \%$ & 1.0 & $5.590(31) \%$ & 1.1 \\
\hline $\operatorname{BR}\left(K_{e 3}\right)$ & $5.078(25) \%$ & 1.2 & $4.98(7) \%$ & 1.3 \\
\hline $\mathrm{BR}(K \mu 3)$ & $3.365(27) \%$ & 1.7 & $3.32(6) \%$ & 1.2 \\
\hline $\operatorname{BR}\left(\pi \pi^{0} \pi^{0}\right)$ & $1.750(24) \%$ & 1.1 & $1.757(24) \%$ & 1.1 \\
\hline $\operatorname{BR}\left(\pi^{0} \pi^{0} e \nu\right)$ & Not in fit & & $2.2(4) \times 10^{-5}$ & 1.0 \\
\hline $\operatorname{BR}\left(\tau_{K^{ \pm}}\right)$ & $12.384(19) \mathrm{ns}$ & 1.7 & $12.385(24) \mathrm{ns}$ & 2.1 \\
\hline
\end{tabular}

Table 16: Results of fit to $K^{ \pm}$BRs and lifetime, with comparison to 2006 PDG fit.

$$
\begin{array}{ccccccc}
1.000 & -0.874 & -0.170 & -0.725 & -0.548 & -0.258 & -0.045 \\
& 1.000 & -0.121 & 0.610 & 0.333 & 0.031 & -0.032 \\
& & 1.000 & -0.100 & -0.074 & 0.055 & 0.273 \\
& & & 1.000 & 0.442 & 0.009 & -0.030 \\
& & & & 1.000 & -0.010 & -0.020 \\
& & & & & 1.000 & 0.010 \\
& & & & & & 1.000
\end{array}
$$

Table 17: Correlation matrix for output parameters of $K^{ \pm}$fit.

\section{Averages of form-factor slopes}

\section{D.1 Procedure}

We work principally with quadratic form-factor slope parametrization. To average the form-factor slopes, a $\chi^{2}$ fit with correlations is performed. Scale factors for the errors are calculated as described in section A. For the fit to the form-factor slopes, since there are no measurements of combinations of the fit parameters, the scale factors can be obtained directly from Eq. (A.2). Because of the high degree of correlation in the measurements of $\lambda^{\prime}$ and $\lambda^{\prime \prime}$, a large scale factor may result in a small change in $\chi^{2}$ from the fits. We therefore report scaled errors only when the value of $\chi^{2} /$ ndf is unsatisfactory.

\section{D.2 Input data}

The data used in the fit are summarized in Table 19. The following notes apply to the table entries.

1. In our combined fits to $K_{e 3}$ and $K_{\mu 3}$ data, we use the averages quoted by KLOE and $\mathrm{KTeV}$ rather than using their $K_{e 3}$ and $K_{\mu 3}$ measurements separately. In any event, our averages of the $K_{e 3}$ and $K_{\mu 3}$ results from each experiment have good values of 


\begin{tabular}{|c|c|c|c|}
\hline Point & Parameter & Source & Pull \\
\hline 1 & $\overline{\tau_{K^{ \pm}}}$ & Koptev '95 & +2.25 \\
\hline 11 & $\operatorname{BR}\left(\pi \pi^{0}\right) / \operatorname{BR}\left(K_{\mu 2}\right)$ & Weissenberg '76 & +1.89 \\
\hline 10 & $\operatorname{BR}\left(\pi \pi^{0}\right) / \operatorname{BR}\left(K_{\mu 2}\right)$ & Usher '92 & +1.70 \\
\hline 5 & $\tau_{K^{ \pm}}$ & Fitch '65 & +1.56 \\
\hline 21 & $\operatorname{BR}\left(K_{\mu 3}\right) / \operatorname{BR}\left(\pi \pi^{0}\right)$ & NA48 '07 & +1.04 \\
\hline 19 & $\operatorname{BR}\left(K_{e 3}\right) / \operatorname{BR}\left(\pi \pi^{0}\right)$ & NA48 '07 & +1.03 \\
\hline 22 & $\operatorname{BR}\left(K_{\mu 3}\right) / \operatorname{BR}\left(K_{e 3}\right)$ & Horie '01 & +0.76 \\
\hline 16 & $\operatorname{BR}\left(K_{e 3}\right) / \operatorname{BR}\left(K_{\mu 2}+\pi \pi^{0}\right)$ & Eschstruth '68 & +0.59 \\
\hline 8 & $\operatorname{BR}\left(K_{\mu 2}\right)$ & KLOE '06 & +0.52 \\
\hline 23 & $\operatorname{BR}\left(K_{\mu 3}\right) / \operatorname{BR}\left(K_{e 3}\right)$ & Heintze '77 & +0.52 \\
\hline 25 & $\operatorname{BR}\left(\pi \pi^{0} \pi^{0}\right)$ & KLOE '04 & +0.52 \\
\hline 12 & $\operatorname{BR}\left(\pi \pi^{0}\right) / \operatorname{BR}\left(K_{\mu 2}\right)$ & Auerbach '67 & +0.46 \\
\hline 24 & $\operatorname{BR}\left(K_{\mu 3}\right) / \operatorname{BR}\left(K_{e 3}\right)$ & Botterill '68 & +0.26 \\
\hline 7 & $\tau_{K^{ \pm}}$ & KLOE '07 & +0.14 \\
\hline 13 & $\Gamma(\pi \pi \pi)$ & Ford '70 & -0.22 \\
\hline 6 & $\tau_{K^{ \pm}}$ & KLOE '06 & -0.21 \\
\hline 3 & $\tau_{K^{ \pm}}$ & Ott '71 & -0.22 \\
\hline 15 & $\operatorname{BR}\left(K_{e 3}\right) / \operatorname{BR}\left(K_{\mu 3}+\pi \pi^{0}+\pi 2 \pi^{0}\right)$ & Sher '03 & -0.26 \\
\hline 2 & $\tau_{K^{ \pm}}$ & Koptev '95 & -0.38 \\
\hline 17 & $\operatorname{BR}\left(K_{e 3}\right) / \operatorname{BR}\left(K_{\mu 2}+\pi \pi^{0}\right)$ & Cester '66 & -0.67 \\
\hline 20 & $\operatorname{BR}\left(K_{e 3}\right) / \operatorname{BR}\left(\pi \pi^{0}\right)$ & ISTRA '07 & -0.74 \\
\hline 26 & $\operatorname{BR}\left(\pi \pi^{0} \pi^{0}\right) / \operatorname{BR}(\pi \pi \pi)$ & Bisi '65 & -1.07 \\
\hline 14 & $\operatorname{BR}\left(K_{e 3}\right)$ & KLOE '07 & -2.13 \\
\hline 4 & $\tau_{K^{ \pm}}$ & Lobkowicz '71 & -3.10 \\
\hline 20 & $\operatorname{BR}\left(K_{\mu 3}\right)$ & KLOE ’07 & -3.41 \\
\hline
\end{tabular}

Table 18: Pull values for input data used in fit to $K^{ \pm}$BRs and lifetime.

$\chi^{2} /$ ndf and confirm the results quoted by the experiments, including the correlation coefficients.

2. The exact value of $\rho\left(\lambda_{+}^{\prime}, \lambda_{+}^{\prime \prime}\right)$ is not available for the NA48 $K_{e 3}$ measurement. NA48 and PDG together estimated $\rho=-0.88$; this value appears in the 2006 PDG listings [43]. For use with Eq. (2.5), we put $\lambda_{+}^{\prime \prime}=2 \lambda_{+}^{\prime \prime(\mathrm{NA} 48)}$.

3. An official value of $\rho\left(\lambda_{+}^{\prime}, \lambda_{+}^{\prime \prime}\right)$ is not available for the ISTRA $+K_{e 3}$ measurement; the value in the Table was obtained directly from the collaboration. For use with Eq. (2.5), we put $\lambda_{+}^{\prime}=C \lambda_{+}^{\prime(\text { ISTRA) }}$ and $\lambda_{+}^{\prime \prime}=2 C^{2} \lambda_{+}^{\prime \prime(\text { ISTRA) }}$, with $C=\left(m_{\pi^{+}} / m_{\pi^{0}}\right)^{2}=$ 1.069223 .

4. Systematic errors for the ISTRA+ quadratic fit results for $K_{\mu 3}$ are not given in Ref. [53]; the errors in the table are statistical only. Nor are the correlation coefficients available; these have been obtained directly from the collaboration. For use with 
Eq. (2.5), $\lambda_{+}^{\prime}$ and $\lambda_{+}^{\prime \prime}$ are converted as above; we also put $\lambda_{0}=C \lambda_{0}^{(\text {ISTRA) }}$. Finally, we note that no information concerning the treatment of radiative corrections is given in Ref. [53]. Failure to account for radiative effects could result in a noticeable systematic shift in the slope results.

For the KLOE and $\mathrm{KTeV}$ form-factor slope measurements, the correlation coefficients apply to the total errors (statistical and systematic). For the ISTRA + and NA48 $K_{\mu 3}$ slopes, the correlation coefficients appear to apply to the statistical errors. In our fits, we assume that the correlation coefficients apply to the total errors on the form-factor slopes (statistical and systematic). This approximation is motivated as follows. In general, the systematic errors are estimated by varying analysis parameters and refitting. In that case, the statistical correlations naturally present will also affect the excursions due to systematic variations, see Appendix $\mathrm{E}$.

\section{D.3 Fit results for $K_{\ell 3}$ slopes excluding NA48 $K_{\mu 3}$ data}

The result of our fit to all data is presented in Table 7. As discussed in Sec. 3.5.2, the NA48 $K_{\mu 3}$ form-factor slope measurements are in contrast with the results from the other experiments. As an exercise, we fit all results in Table 19 except the NA48 measurement of the $K_{\mu 3}$ slopes [54]. The results are shown in Table 20. The first column of the table gives the results of the fit to all other measurements from KLOE; the second gives the results of the fit to the $K_{L}$ measurements from $\mathrm{KLOE}, \mathrm{KTeV}$, and the $K_{L e 3}$ measurement from NA48.

The evaluations of the phase-space integrals for all four modes are listed in each case. Correlations are fully accounted for, both in the fits and in the evaluation of the integrals. The values of $\chi^{2} /$ ndf do not raise any significant concerns about the compatibility of the input data. The fit to all data gives $\chi^{2} / \mathrm{ndf}=12.6 / 10(25.0 \%)$.

The evaluations of the phase-space integrals for all four modes are listed in each case. Correlations are fully accounted for, both in the fits and in the evaluation of the integrals. The values of $\chi 2 /$ ndf do not raise any significant concerns about the compatibility of the input data. The fit to all data

\begin{tabular}{lcc}
\hline \hline & $K_{L}$ and $K^{-}$ & $K_{L}$ only \\
\hline Measurements & 13 & 8 \\
$\chi^{2} / \mathrm{ndf}$ & $13 / 9(24.9 \%)$ & $9 / 5(12.3 \%)$ \\
$\lambda_{+}^{\prime} \times 10^{3}$ & $25.0 \pm 0.8$ & $24.5 \pm 1.1$ \\
$\lambda_{+}^{\prime \prime} \times 10^{3}$ & $1.6 \pm 0.4$ & $1.8 \pm 0.4$ \\
$\lambda_{0} \times 10^{3}$ & $16.0 \pm 0.8$ & $14.8 \pm 1.1$ \\
$\rho\left(\lambda_{+}^{\prime}, \lambda_{+}^{\prime \prime}\right)$ & -0.94 & -0.95 \\
$\rho\left(\lambda_{+}^{\prime}, \lambda_{0}\right)$ & +0.26 & +0.28 \\
$\rho\left(\lambda_{+}^{\prime \prime}, \lambda_{0}\right)$ & -0.37 & -0.38 \\
$I\left(K_{e 3}^{0}\right)$ & $0.15459(20)$ & $0.15446(27)$ \\
$I\left(K_{e 3}^{ \pm}\right)$ & $0.15894(21)$ & $0.15881(28)$ \\
$I\left(K_{\mu 3}^{0}\right)$ & $0.10268(20)$ & $0.10236(28)$ \\
$I\left(K_{\mu 3}^{ \pm}\right)$ & $0.10559(20)$ & $0.10532(29)$ \\
$\rho\left(I_{e 3}, I_{\mu 3}\right)$ & +0.59 & +0.62 \\
\hline \hline
\end{tabular}

Table 20: Averages of quadratic fit results for $K_{e 3}$ and $K_{\mu 3}$ slopes, excluding new $K_{\mu 3}$ data from NA48. 


\section{E. Error estimates}

It is quite easy to estimate the ideal error in the measurements of a set of parameters $\mathbf{p}=\left(p_{1}, p_{2}, \ldots p_{n}\right)$ from fitting some distribution function to experimentally determined spectra. Let $F(\mathbf{p}, x)$ be a probability density function, PDF, where $\mathbf{p}$ is some parameter vector, which we want to determine and $x$ is a running variable, like $t$. The inverse of the covariance matrix for the maximum likelihood estimate of the parameters is given by [100]:

$$
\left(\mathbf{G}^{-1}\right)_{i j}=-\frac{\partial^{2} \ln L}{\partial p_{i} \partial p_{j}}
$$

from which, for $N$ events, it trivially follows:

$$
\left(\mathbf{G}^{-1}\right)_{i j}=N \int \frac{1}{F} \frac{\partial F}{\partial p_{i}} \frac{\partial F}{\partial p_{j}} \mathrm{~d} v
$$

with $\mathrm{d} v$ the appropriate volume element. We use in the following the above relation to estimate the errors on the FF parameters for one and two parameters expression of the FFs $\tilde{f}_{+}(t)$ and $\tilde{f}_{0}(t)$. The errors in any realistic experiment will be larger than our estimates, typically two to three times. The above estimates are useful for the understanding of the problems in the determination of the parameters in question. The elements of $\mathrm{G}$ depend on the values of the parameters. In the case of the form factors, the errors on the $\lambda$ parameters change insignificantly for $10 \%$ changes of the parameters. In other words the errors do not depend on the data being fitted and the correlations apply also to the systematic part of the errors.

\section{E.1 $K_{e 3}$ decays}

For a quadratic FF, $\tilde{f}(t)=1+\lambda_{+}^{\prime}\left(t / m^{2}\right)+\left(\lambda_{+}^{\prime \prime} / 2\right)\left(t / m^{2}\right)^{2}$, the inverse of the covariance matrix $\mathbf{G}_{+}^{-1}$, the covariance matrix $\mathbf{G}_{+}$and the correlation matrix are:

$$
N\left(\begin{array}{cc}
5.937 & 13.867 \\
13.867 & 36.2405
\end{array}\right), \quad \frac{1}{N}\left(\begin{array}{cc}
1.258^{2} & -0.606 \\
-0.606 & 0.509^{2}
\end{array}\right), \quad\left(\begin{array}{cc}
1 & -.945 \\
1
\end{array}\right)
$$

The square root of the diagonal elements of $\mathbf{G}_{+}$gives the errors, which for one million events are $\delta \lambda_{+}^{\prime}=0.00126, \delta \lambda_{+}^{\prime \prime}=0.00051$. The correlation is very close to -1 , meaning that, because of statistical fluctuation of the bin counts, a fit will trade $\lambda_{+}^{\prime}$ for $\lambda_{+}^{\prime \prime}$ and that the errors are enlarged. A fit for a linear FF, $\tilde{f}(t)=1+\lambda_{+}^{\prime}\left(t / m^{2}\right)$ in fact gives $\lambda_{+}^{\prime}=0.029$ instead of 0.025 and an error smaller by $\sim 3$ :

$$
\delta \lambda_{+}^{\prime}=\sqrt{\mathbf{G}_{+}(1,1)}=0.0004 .
$$

A simple rule of thumb is that ignoring a $t^{2}$ term, increases $\lambda_{+}^{\prime}$ by $\sim 3.5 \times \lambda_{+}^{\prime \prime}$. For $K_{e 3}$ decays the presence of a $t^{2}$ term in the FF is firmly established. It is however not fully justified to fit for two parameters connected by the simple relation $\lambda_{+}^{\prime \prime}=2 \times \lambda_{+}^{\prime}{ }^{2}$. The authors of ref. [12] explicitly give an error for their estimate of the coefficient of the $t^{2}$ terms. 


\section{E.2 $K_{\mu 3}$ decays}

The scalar FF only contributes to $K_{\mu 3}$ decays. Dealing with these decays is much harder because: a) - the branching ratio is smaller, resulting in reduced statistics, b) - the $E_{\pi}$ or $t$ range in the decay is smaller, c) - it is in general harder to obtain an undistorted spectrum and d) - more parameters are necessary. This is quite well evidenced by the wide range of answers obtained by different experiments [48, 53, 54, 52]. Assuming that both scalar and vector $\mathrm{FF}$ are given by quadratic polynomials as in Eq. (2.5), ordering the parameters as $\lambda_{0}^{\prime}, \lambda_{0}^{\prime \prime}, \lambda_{+}^{\prime}$ and $\lambda_{+}^{\prime \prime}$, the matrices $\mathbf{G}_{0 \&+}^{-1}$ and $\mathbf{G}_{0 \&+}$, are:

$$
N\left(\begin{array}{cccc}
1.64 & 5.44 & 1.01 & 3.90 \\
5.44 & 18.2 & 3.01 & 12.3 \\
1.01 & 3.01 & 1.47 & 4.24 \\
3.90 & 12.3 & 4.24 & 13.8
\end{array}\right), \frac{1}{N}\left(\begin{array}{cccc}
63.9^{2} & -1200 & -923 & 197 \\
-1200 & 18.8^{2} & 272 & -59 \\
-923 & 272 & 14.8^{2} & -49 \\
197 & -59 & -48 & 3.4^{2}
\end{array}\right)
$$

and the correlations, ignoring the diagonal terms, are:

$$
\left(\begin{array}{ccc}
-0.9996 & -0.974 & 0.91 \\
& 0.978 & -0.919 \\
& & -0.976
\end{array}\right)
$$

All correlations are very close to -1 . In particular the correlations between $\lambda_{0}^{\prime}$ and $\lambda_{0}^{\prime \prime}$ is $-99.96 \%$, reflecting in vary large $\delta \lambda_{0}^{\prime}$ and $\delta \lambda_{0}^{\prime \prime}$ errors. We might ask what the error on $\lambda_{0}^{\prime}$ and $\lambda_{0}^{\prime \prime}$ might be if we had perfect knowledge of $\lambda_{+}^{\prime}$ and $\lambda_{+}^{\prime \prime}$. The inverse covariance matrix is give by the elements $(1,1),(1,2),(2,1)$ and $(2,2)$ of the $\mathbf{G}_{0 \&+}^{-1}$ matrix above. The covariance matrix therefore is :

$$
\mathbf{G}_{0}\left(\lambda_{0}^{\prime}, \lambda_{0}^{\prime \prime} \text { for } \lambda_{+}^{\prime}, \lambda_{+}^{\prime \prime} \text { known }\right)=\frac{1}{N}\left(\begin{array}{cc}
8.2^{2} & -20 \\
-20 & 2.4^{2}
\end{array}\right) .
$$

For one million events we have $\delta \lambda_{0}^{\prime \prime}=0.0024$, about $4 \times$ the expected value of $\lambda_{0}^{\prime \prime}$. In other words $\lambda_{0}^{\prime \prime}$ is likely to be never measurable. It is however a mistake to assume a scalar FF linear in $t$, because the coefficient of $t$ will absorb the coefficient of a $t^{2}$ term, again multiplied by $\sim 3.5$. Thus a real value $\lambda_{0}^{\prime}=0.014$ is shifted by the fit to 0.017 , having used the parametrization of Ref. [12].

\section{E.3 From the linear to the dispersive parametrization}

The results on the FFs obtained with the linear parametrization can be used to determine the parameter of the dispersive parametrization. As shown in the previous section the correlation between $\lambda_{0}^{\prime}$ and $\lambda_{0}^{\prime \prime}$ is close to -1 and any fit to $\lambda_{0}^{\prime}, \lambda_{0}^{\prime \prime}$ form $K_{\mu 3}$ decays will give values satisfying the relation:

$$
\lambda_{0}^{\prime \prime}=\tan \phi \lambda_{0}^{\prime}+B
$$

$\tan \phi$ is independent on the number of events of the experiment

$$
\tan 2 \phi=\frac{2 \rho_{1,2} \sigma_{2} \sigma_{1}}{\sigma_{2}^{2}-\sigma_{1}^{2}} \text { with } 1=\lambda_{0}^{\prime}, 2=\lambda_{0}^{\prime \prime}
$$




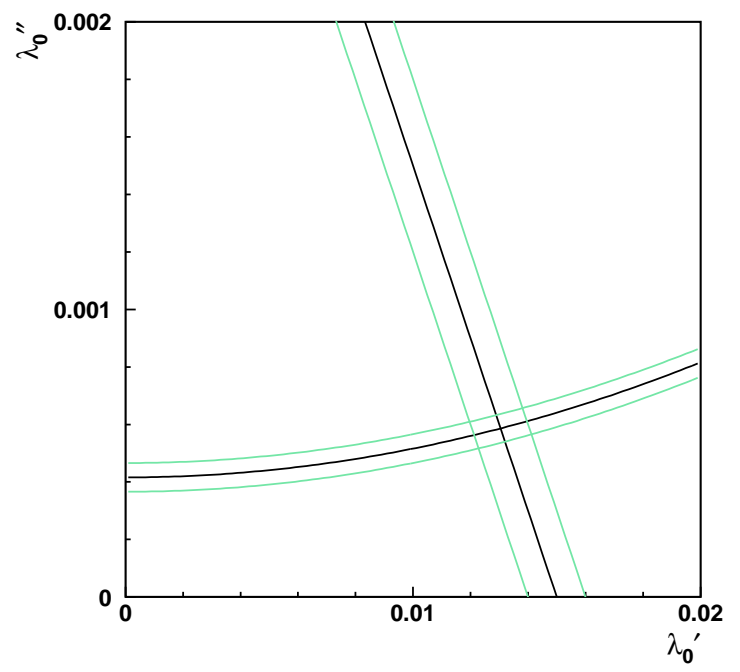

Figure 12: Linear parametrization extrapolation along correlation line and relation from dispersive parametrization.

and amounts to -0.3 . B can be determined from the experimental results for $\lambda_{0}$ obtained using the linear parametrization $\left(\lambda_{0}^{\prime \prime}=0\right)$.

Therefore we can translate the results for $\lambda_{0}$ in any new parametrization with only one parameter with almost negligible 3 th order term. In particular the dispersive parametrization gives:

$$
\lambda_{0}^{\prime \prime}=\lambda_{0}^{\prime 2}+(4.16 \pm 0.50) \times 10^{-4}
$$

the procedure is shown in figure 12 for $\lambda_{0}=(15 \pm 1) \times 10^{-3}$. 


\begin{tabular}{|c|c|c|c|c|c|c|c|c|}
\hline Experiment & $\overline{\lambda_{+}^{\prime} \times 10^{3}}$ & $\lambda_{+}^{\prime \prime} \times 10^{3}$ & $\lambda_{0} \times 10^{3}$ & 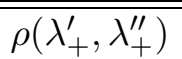 & 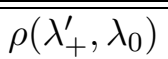 & 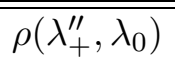 & Analysis & Note \\
\hline$\overline{\mathrm{KLOE}} K_{L} e 3[49]$ & $25.5 \pm 1.8$ & $1.4 \pm 0.8$ & & -0.95 & & & $t$ from $K_{S} \rightarrow \pi^{+} \pi^{-}$ & \\
\hline $\mathrm{KLOE} K_{L} \mu 3[52]$ & $22.3 \pm 10.5$ & $4.8 \pm 5.2$ & $9.1 \pm 6.5$ & -0.97 & +0.81 & -0.91 & $E_{\nu}^{*}$ & \\
\hline KLOE $K_{L} e 3-\mu 3[52]$ & $25.6 \pm 1.8$ & $1.5 \pm 0.8$ & $15.4 \pm 2.2$ & -0.95 & +0.29 & -0.38 & average & 1 \\
\hline $\mathrm{KTeV} K_{L} e 3[48]$ & $21.67 \pm 1.99$ & $2.87 \pm 0.78$ & & -0.97 & & & $t_{\perp}^{\pi}$ & \\
\hline $\mathrm{KTeV} K_{L} \mu 3[48]$ & $17.03 \pm 3.65$ & $4.43 \pm 1.49$ & $12.81 \pm 1.83$ & -0.96 & +0.65 & -0.75 & $\left(t_{\perp}^{\mu}, \bar{M}_{\pi \mu}\right)$ & \\
\hline $\mathrm{KTeV} K_{L} e 3-\mu 3[48]$ & $20.64 \pm 1.75$ & $3.20 \pm 0.69$ & $13.72 \pm 1.31$ & -0.97 & +0.34 & -0.44 & average & 1 \\
\hline NA48 $K_{L} e 3[51]$ & $28.0 \pm 2.4$ & $0.4 \pm 0.9$ & & $-0.88^{*}$ & & & $\left(E_{\nu}^{*}, t_{\text {low }}, t_{\text {high }}\right)$ & 2 \\
\hline NA48 $K_{L} \mu 3[54]$ & $20.5 \pm 3.3$ & $2.6 \pm 1.3$ & $9.5 \pm 1.4$ & -0.96 & +0.63 & -0.73 & $(y, z)_{\text {low }}$ & \\
\hline ISTRA $+K^{-} e 3[50]$ & $24.85 \pm 1.66$ & $1.92 \pm 0.94$ & & $-0.95 *$ & & & $(y, z)_{2 \mathrm{Cfit}}$ & 3 \\
\hline ISTRA $+K^{-} \mu 3[53]$ & $22.99 \pm 6.42^{*}$ & $2.29 \pm 2.29^{*}$ & $17.11 \pm 2.25^{*}$ & $-0.82^{*}$ & $-0.12^{*}$ & $-0.41^{*}$ & $(y, z)_{2 \mathrm{Cfit}}$ & 4 \\
\hline
\end{tabular}

$\square \square \square$ Table 19. Measurements of $K_{\ell 3}$ form-factor slopes. Values marked with an asterisk involve additional assumptions; see notes in text. 